Supporting Information for

\title{
New Helical Foldamers: Heterogeneous Backbones with 1:2 and 2:1 $\alpha: \beta$-Amino Acid Residue Patterns
}

Margaret A. Schmitt, Soo Hyuk Choi, Ilia A. Guzei, and Samuel H. Gellman*

\section{Peptide synthesis and purification}

$\alpha / \beta$-Peptides 1 and $\mathbf{2}$ were synthesized manually using HBTU/HOBt activation on NovaSyn TGR resin or on a Synergy automated peptide synthesizer. $\beta$-Amino acids (FmocACPC-OH and Fmoc-APC(Boc)-OH) were synthesized from $\beta$-keto esters [1]. Fmoc- $\alpha$ amino acids were purchased from EMD Biosciences. Crude peptides $\mathbf{1}$ and $\mathbf{2}$ were purified by RP-HPLC on a $\mathrm{C}_{4}$-silica column using a linear gradient of acetonitrile in water.

NMR Chemical Shift Data (ppm):

\begin{tabular}{|c|c|c|c|c|}
\hline$\alpha / \beta$-Peptide 1 & $\mathrm{HN}$ & $H \alpha$ & $\mathrm{H} \boldsymbol{\beta}$ & Other \\
\hline $\mathrm{N}$-terminus & & & & $\mathrm{CH}_{3}: 1.98$ \\
\hline ACPC 1 & 8.44 & 2.41 & 4.06 & $\begin{array}{l}\mathrm{H} \gamma: 2.03,1.65 \\
\mathrm{H} \varepsilon: 1.93,1.81 \\
\mathrm{H} \delta: ?\end{array}$ \\
\hline APC 2 & 8.83 & 3.07 & 4.74 & $\begin{array}{l}\mathrm{H} \gamma: 3.61,3.27 \\
\mathrm{H} \varepsilon: 3.61,3.50\end{array}$ \\
\hline Tyr 3 & 8.96 & 4.29 & $2.98,2.89$ & HAr: $7.05,6.67$ \\
\hline APC 4 & 8.69 & 3.12 & 4.75 & $\mathrm{H}_{\gamma}$ and $\mathrm{H}_{\varepsilon}: 3.62,3.56$ \\
\hline ACPC 5 & 8.08 & 2.68 & 4.32 & $\begin{array}{l}\mathrm{H} \gamma: 2.01,1.72 \\
\mathrm{H} \varepsilon: 1.93,1.86 \\
\mathrm{H} \delta: 1.79\end{array}$ \\
\hline Lys 6 & 8.36 & 4.26 & $1.89,1.70$ & $\begin{array}{l}{ }_{\varepsilon} \mathrm{NH}_{2}: 7.83 \\
\mathrm{H} \varepsilon: 2.89 \\
\mathrm{H} \delta: 1.65 \\
\mathrm{H} \gamma: 1.52,1.46\end{array}$ \\
\hline C-terminus & 7.97, & & & \\
\hline
\end{tabular}




\begin{tabular}{|c|c|c|c|c|}
\hline$\alpha / \beta$-Peptide 2 & HN & $H \alpha$ & $H \beta$ & Other \\
\hline $\mathrm{N}$-terminus & & & & $\mathrm{CH}_{3}: 1.93$ \\
\hline ACPC 1 & 8.45 & 2.59 & 4.22 & $\begin{array}{l}\mathrm{H}_{\gamma} \text { and } \mathrm{H}_{\varepsilon}: 2.04 \\
\mathrm{H} \delta \text { and } \mathrm{H}_{\varepsilon}: 1.75 \\
\mathrm{H} \gamma \text { and } \mathrm{H} \delta: 1.64\end{array}$ \\
\hline Lys 2 & 8.71 & 4.01 & 1.79 & $\begin{array}{l}\mathrm{H} \varepsilon: 2.92 \\
\mathrm{H} \delta: 1.66 \\
\mathrm{H} \gamma: 1.51,1.45\end{array}$ \\
\hline Ala 3 & 8.37 & 4.22 & 1.40 & \\
\hline APC 4 & 8.22 & 3.26 & 4.60 & $\begin{array}{l}\mathrm{H}_{\gamma}: 3.58,3.30 \\
\mathrm{H}_{\varepsilon}: 3.58,3.43\end{array}$ \\
\hline Tyr 5 & 8.56 & 4.31 & $3.08,2.95$ & HAr: $7.06,6.67$ \\
\hline Gln 6 & 8.23 & 4.18 & 2.11 & $\begin{array}{l}\gamma \mathrm{CO}=\mathrm{NH}_{2}: 7.65,6.98 \\
\mathrm{H} \gamma: 2.34,2.26\end{array}$ \\
\hline ACPC 7 & 7.74 & 2.67 & 4.25 & $\begin{array}{l}\mathrm{H} \gamma: 2.01,1.64 \\
\mathrm{H} \varepsilon: 1.95,1.84 \\
\mathrm{H} \delta: 1.76\end{array}$ \\
\hline C-terminus & 7.60 , & & & \\
\hline
\end{tabular}

Backbone-Backbone NOEs between Residues Non-Adjacent in Sequence in Heterogeneous Backbone $\alpha \beta$-Peptides 1 and 2:

$\alpha / \beta$-Peptide 1

\begin{tabular}{lll}
\hline NOE Assignment & CS1 $(\mathrm{ppm})$ & CS2 $(\mathrm{ppm})$ \\
\hline Tyr3HN-ACPC1HB & 8.93 & 4.06 \\
Tyr3HN-NtermAc & 8.93 & 1.98 \\
APC2HN-NtermAc & 8.82 & 1.98 \\
APC4HN-ACPC1HB & 8.69 & 4.06 \\
APC4HN-APC2HA & 8.69 & 3.07 \\
Lys6HN-APC4HB & 8.36 & 4.75 \\
CtermHN-APC4HB & 7.96 & 4.75 \\
CtermHN-ACPC5HB & 7.96 & 4.32 \\
ACPC1HB-APC4HA & 4.06 & 3.12 \\
ACPC5HA-CtermHN & 2.68 & 7.97 \\
ACPC5HA-APC2HB & 2.68 & 4.74 \\
\hline
\end{tabular}


$\alpha / \beta$-Peptide 2

\begin{tabular}{lll}
\hline NOE Assignment & CS1 $(\mathrm{ppm})$ & CS2 $(\mathrm{ppm})$ \\
\hline Tyr5HN-Lys2HA & 8.54 & 4.01 \\
Ala3HN-NtermAc & 8.38 & 1.93 \\
APC4HN-Lys2HA & 8.22 & 4.01 \\
ACPC7HN-APC4HB & 7.74 & 4.60 \\
CtermHN-Tyr5HA & 7.60 & 4.31 \\
APC4HB-ACPC7HA & 4.60 & 2.67 \\
Ala3HA-Tyr5HN & 4.22 & 8.54 \\
ACPC1HB-APC4HA & 4.22 & 3.26 \\
\hline
\end{tabular}




\section{Crystallographic Experimental Section}

The crystal structures have been deposited at the Cambridge Crystallographic Data Centre with deposition number CCDC 289104 (3) and 294736 (4).

\section{Peptide synthesis and crystallization}

$\alpha / \beta$-Peptides 3 and $\mathbf{4}$ were prepared by conventional solution-phase peptide synthesis using EDCI/DMAP activation. Boc-ACPC-OH was synthesized as the literature procedure [1]. Boc-Phe-OH and Boc-Aib-OH were purchased from Fluka and Chem-Impex. X-ray quality crystals were grown from chloroform/diethyl ether mixtures.

\section{Data collection for 3}

The X-ray diffraction data of $\mathbf{3}$ were acquired at the Advanced Photon Source (Argonne National Laboratory).

A colorless crystal with approximate dimensions $0.45 \times 0.33 \times 0.21 \mathrm{~mm}^{3}$ was mounted in a stream of cold nitrogen at $100(2) \mathrm{K}$ and centered in the X-ray beam by using a video camera.

\section{Structure Solution and Refinement of 3}

The systematic absences in the diffraction data were consistent for the space group $P 6_{5}$ that yielded chemically reasonable and computationally stable results of refinement [2].

A successful solution by the direct methods provided most non-hydrogen atoms from the E-map. The remaining non-hydrogen atoms were located in an alternating series of leastsquares cycles and difference Fourier maps. All non-hydrogen atoms were refined with isotropic displacement coefficients. All hydrogen atoms were included in the structure factor calculation at idealized positions and were allowed to ride on the neighboring atoms with relative isotropic displacement coefficients.

There are also two molecules of solvate chloroform per organic molecule in the lattice. The absolute configuration was assigned from the known synthetic procedure. 
The final least-squares refinement of 312 parameters against 10606 data resulted in residuals $R$ (based on $F^{2}$ for $I \geq 2 \sigma$ ) and $w R$ (based on $F^{2}$ for all data) of 0.1581 and 0.3981 , respectively.

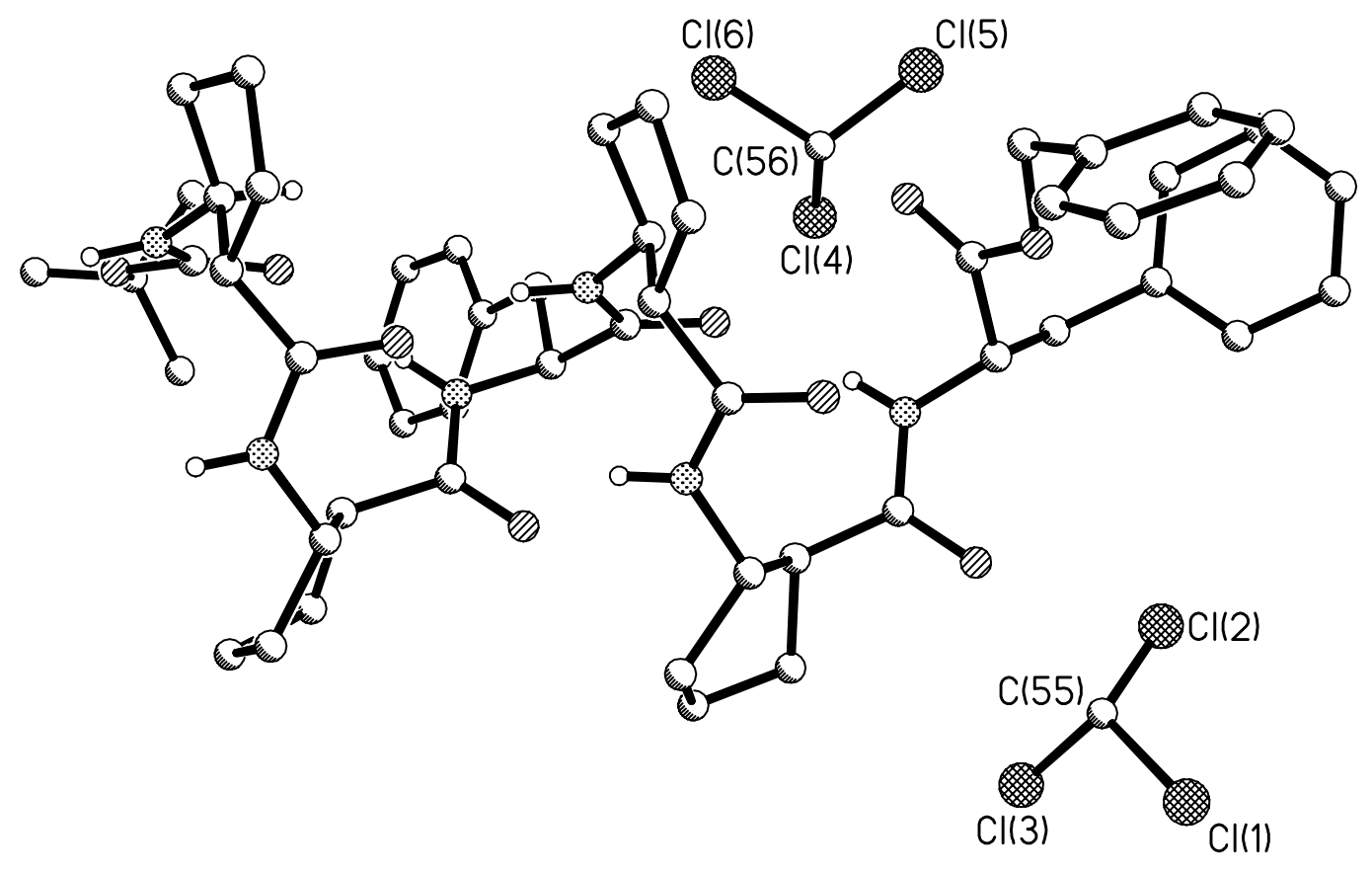

Figure S1. The content of the asymmetric unit for 3 . 


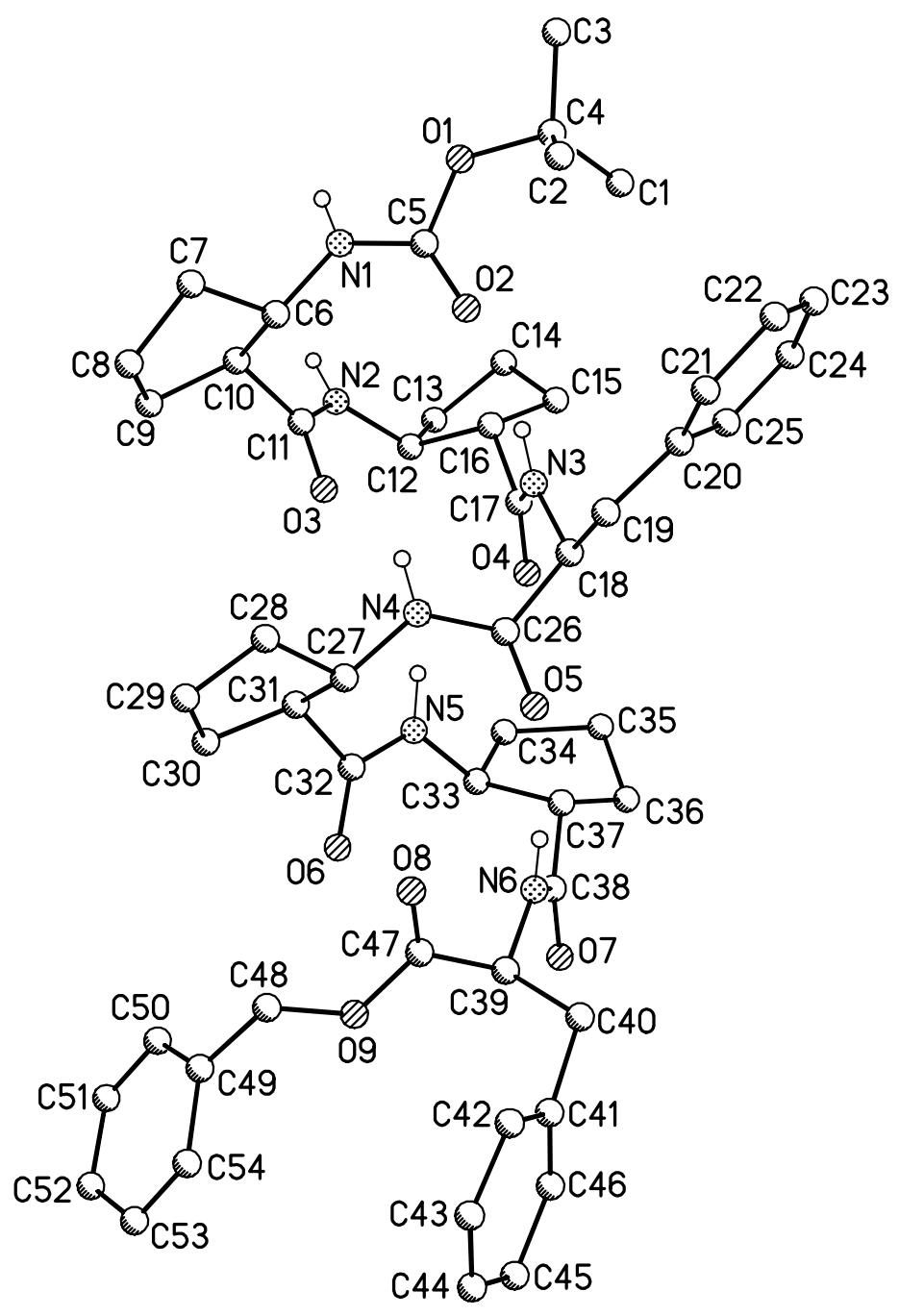

Figure S2. Solid state conformation of 3. All $\mathrm{H}$ atoms except those on the $\mathrm{N}$ atoms are omitted. 
Table S1. Crystal data and structure refinement for 3 .

\begin{tabular}{|c|c|c|}
\hline Empirical formula & $\mathrm{C}_{56} \mathrm{H}_{72} \mathrm{Cl}_{6} \mathrm{~N}_{6} \mathrm{O}_{9}$ & \\
\hline Formula weight & 1185.90 & \\
\hline Temperature & $100(2) \mathrm{K}$ & \\
\hline Wavelength & $0.71073 \AA$ & \\
\hline Crystal system & Hexagonal & \\
\hline Space group & $\mathrm{P}_{5}$ & \\
\hline Unit cell dimensions & $\mathrm{a}=21.321(3) \AA$ & $\alpha=90^{\circ}$. \\
\hline & $\mathrm{b}=21.321(3) \AA$ & $\beta=90^{\circ}$. \\
\hline & $\mathrm{c}=23.846(4) \AA$ & $\gamma=120^{\circ}$ \\
\hline Volume & $9388(2) \AA^{3}$ & \\
\hline Z & 6 & \\
\hline Density (calculated) & $1.259 \mathrm{Mg} / \mathrm{m}^{3}$ & \\
\hline Absorption coefficient & $0.330 \mathrm{~mm}^{-1}$ & \\
\hline $\mathrm{F}(000)$ & 3744 & \\
\hline Crystal size & $0.47 \times 0.39 \times 0.19$ & \\
\hline Theta range for data collection & 2.03 to $24.82^{\circ}$ & \\
\hline Index ranges & $-25<=\mathrm{h}<=25,-25$ & $<=1<=27$ \\
\hline Reflections collected & 132471 & \\
\hline Independent reflections & $10606[\mathrm{R}(\mathrm{int})=0$ & \\
\hline Completeness to theta $=24.82^{\circ}$ & $99.4 \%$ & \\
\hline Absorption correction & Multi-scan with S & \\
\hline Max. and min. transmission & 0.9399 and 0.860 & \\
\hline Refinement method & Full-matrix least-s & \\
\hline Data / restraints / parameters & $10606 / 1 / 312$ & \\
\hline Goodness-of-fit on $\mathrm{F}^{2}$ & 1.389 & \\
\hline Final $R$ indices $[\mathrm{I}>2 \operatorname{sigma}(\mathrm{I})]$ & $\mathrm{R} 1=0.1581, \mathrm{wR} 2$ & \\
\hline $\mathrm{R}$ indices (all data) & $\mathrm{R} 1=0.1928, \mathrm{wR} 2$ & \\
\hline Absolute structure parameter & $0.38(18)-$ assigne & hesis \\
\hline Largest diff. peak and hole & 1.229 and -1.283 & \\
\hline
\end{tabular}


Table S2. Torsion angles $\left[^{\circ}\right]$ for 3.

\begin{tabular}{|c|c|c|c|}
\hline $\mathrm{C}(5)-\mathrm{O}(1)-\mathrm{C}(4)-\mathrm{C}(3)$ & $-171.1(9)$ & $\mathrm{C}(18)-\mathrm{C}(19)-\mathrm{C}(20)-\mathrm{C}(21)$ & 169.3(9) \\
\hline $\mathrm{C}(5)-\mathrm{O}(1)-\mathrm{C}(4)-\mathrm{C}(2)$ & $-52.7(12)$ & $\mathrm{C}(18)-\mathrm{C}(19)-\mathrm{C}(20)-\mathrm{C}(25)$ & $-11.3(14)$ \\
\hline $\mathrm{C}(5)-\mathrm{O}(1)-\mathrm{C}(4)-\mathrm{C}(1)$ & $70.3(12)$ & $\mathrm{C}(25)-\mathrm{C}(20)-\mathrm{C}(21)-\mathrm{C}(22)$ & $3.2(16)$ \\
\hline $\mathrm{C}(6)-\mathrm{N}(1)-\mathrm{C}(5)-\mathrm{O}(2)$ & $19.8(14)$ & $C(19)-C(20)-C(21)-C(22)$ & $-177.4(10)$ \\
\hline $\mathrm{C}(6)-\mathrm{N}(1)-\mathrm{C}(5)-\mathrm{O}(1)$ & $-162.3(8)$ & $C(20)-C(21)-C(22)-C(23)$ & $-0.2(18)$ \\
\hline $\mathrm{C}(4)-\mathrm{O}(1)-\mathrm{C}(5)-\mathrm{O}(2)$ & $-10.7(13)$ & $C(21)-C(22)-C(23)-C(24)$ & $-2.8(17)$ \\
\hline $\mathrm{C}(4)-\mathrm{O}(1)-\mathrm{C}(5)-\mathrm{N}(1)$ & $171.4(8)$ & $C(22)-C(23)-C(24)-C(25)$ & $2.8(18)$ \\
\hline C(5)-N(1)-C(6)-C(7) & $117.8(9)$ & $C(23)-C(24)-C(25)-C(20)$ & $0.3(17)$ \\
\hline C(5)-N(1)-C(6)-C(10) & $-122.5(9)$ & $\mathrm{C}(21)-\mathrm{C}(20)-\mathrm{C}(25)-\mathrm{C}(24)$ & $-3.2(16)$ \\
\hline $\mathrm{N}(1)-\mathrm{C}(6)-\mathrm{C}(7)-\mathrm{C}(8)$ & $166.9(8)$ & $\mathrm{C}(19)-\mathrm{C}(20)-\mathrm{C}(25)-\mathrm{C}(24)$ & $177.4(10)$ \\
\hline $\mathrm{C}(10)-\mathrm{C}(6)-\mathrm{C}(7)-\mathrm{C}(8)$ & $42.1(10)$ & $\mathrm{C}(27)-\mathrm{N}(4)-\mathrm{C}(26)-\mathrm{O}(5)$ & $0.4(14)$ \\
\hline $\mathrm{C}(6)-\mathrm{C}(7)-\mathrm{C}(8)-\mathrm{C}(9)$ & $-34.4(11)$ & $C(27)-N(4)-C(26)-C(18)$ & $-176.2(8)$ \\
\hline C(7)-C(8)-C(9)-C(10) & $13.2(11)$ & $\mathrm{N}(3)-\mathrm{C}(18)-\mathrm{C}(26)-\mathrm{O}(5)$ & $160.3(8)$ \\
\hline $\mathrm{C}(8)-\mathrm{C}(9)-\mathrm{C}(10)-\mathrm{C}(11)$ & $130.7(9)$ & $\mathrm{C}(19)-\mathrm{C}(18)-\mathrm{C}(26)-\mathrm{O}(5)$ & $-74.8(10)$ \\
\hline $\mathrm{C}(8)-\mathrm{C}(9)-\mathrm{C}(10)-\mathrm{C}(6)$ & $12.3(10)$ & $\mathrm{N}(3)-\mathrm{C}(18)-\mathrm{C}(26)-\mathrm{N}(4)$ & $-23.1(12)$ \\
\hline $\mathrm{N}(1)-\mathrm{C}(6)-\mathrm{C}(10)-\mathrm{C}(11)$ & $77.9(10)$ & $\mathrm{C}(19)-\mathrm{C}(18)-\mathrm{C}(26)-\mathrm{N}(4)$ & $101.8(9)$ \\
\hline $\mathrm{C}(7)-\mathrm{C}(6)-\mathrm{C}(10)-\mathrm{C}(11)$ & $-155.9(9)$ & $\mathrm{C}(26)-\mathrm{N}(4)-\mathrm{C}(27)-\mathrm{C}(31)$ & $-112.5(10)$ \\
\hline N(1)-C(6)-C(10)-C(9) & $-159.3(8)$ & $\mathrm{C}(26)-\mathrm{N}(4)-\mathrm{C}(27)-\mathrm{C}(28)$ & $131.3(10)$ \\
\hline C(7)-C(6)-C(10)-C(9) & $-33.1(10)$ & $\mathrm{N}(4)-\mathrm{C}(27)-\mathrm{C}(28)-\mathrm{C}(29)$ & $159.3(10)$ \\
\hline $\mathrm{C}(12)-\mathrm{N}(2)-\mathrm{C}(11)-\mathrm{O}(3)$ & $-11.2(14)$ & $\mathrm{C}(31)-\mathrm{C}(27)-\mathrm{C}(28)-\mathrm{C}(29)$ & $37.1(12)$ \\
\hline $\mathrm{C}(12)-\mathrm{N}(2)-\mathrm{C}(11)-\mathrm{C}(10)$ & $166.9(8)$ & $\mathrm{C}(27)-\mathrm{C}(28)-\mathrm{C}(29)-\mathrm{C}(30)$ & $-22.1(13)$ \\
\hline $\mathrm{C}(9)-\mathrm{C}(10)-\mathrm{C}(11)-\mathrm{O}(3)$ & $-48.5(13)$ & $\mathrm{C}(28)-\mathrm{C}(29)-\mathrm{C}(30)-\mathrm{C}(31)$ & $-1.5(13)$ \\
\hline $\mathrm{C}(6)-\mathrm{C}(10)-\mathrm{C}(11)-\mathrm{O}(3)$ & $66.1(12)$ & $C(29)-C(30)-C(31)-C(32)$ & $146.8(10)$ \\
\hline $\mathrm{C}(9)-\mathrm{C}(10)-\mathrm{C}(11)-\mathrm{N}(2)$ & $133.4(9)$ & $C(29)-C(30)-C(31)-C(27)$ & $24.5(12)$ \\
\hline $\mathrm{C}(6)-\mathrm{C}(10)-\mathrm{C}(11)-\mathrm{N}(2)$ & $-112.0(10)$ & $\mathrm{N}(4)-\mathrm{C}(27)-\mathrm{C}(31)-\mathrm{C}(30)$ & $-160.1(9)$ \\
\hline $\mathrm{C}(11)-\mathrm{N}(2)-\mathrm{C}(12)-\mathrm{C}(13)$ & $175.4(9)$ & $\mathrm{C}(28)-\mathrm{C}(27)-\mathrm{C}(31)-\mathrm{C}(30)$ & $-37.7(11)$ \\
\hline $\mathrm{C}(11)-\mathrm{N}(2)-\mathrm{C}(12)-\mathrm{C}(16)$ & $-67.5(11)$ & $\mathrm{N}(4)-\mathrm{C}(27)-\mathrm{C}(31)-\mathrm{C}(32)$ & $74.9(11)$ \\
\hline $\mathrm{N}(2)-\mathrm{C}(12)-\mathrm{C}(13)-\mathrm{C}(14)$ & $100.3(11)$ & $\mathrm{C}(28)-\mathrm{C}(27)-\mathrm{C}(31)-\mathrm{C}(32)$ & $-162.8(9)$ \\
\hline $\mathrm{C}(16)-\mathrm{C}(12)-\mathrm{C}(13)-\mathrm{C}(14)$ & $-19.1(12)$ & $\mathrm{C}(33)-\mathrm{N}(5)-\mathrm{C}(32)-\mathrm{O}(6)$ & $-4.3(14)$ \\
\hline$C(12)-C(13)-C(14)-C(15)$ & $39.4(13)$ & $\mathrm{C}(33)-\mathrm{N}(5)-\mathrm{C}(32)-\mathrm{C}(31)$ & $174.1(8)$ \\
\hline $\mathrm{C}(13)-\mathrm{C}(14)-\mathrm{C}(15)-\mathrm{C}(16)$ & $-44.3(13)$ & $\mathrm{C}(30)-\mathrm{C}(31)-\mathrm{C}(32)-\mathrm{O}(6)$ & $-18.5(14)$ \\
\hline $\mathrm{C}(14)-\mathrm{C}(15)-\mathrm{C}(16)-\mathrm{C}(17)$ & $153.7(10)$ & $\mathrm{C}(27)-\mathrm{C}(31)-\mathrm{C}(32)-\mathrm{O}(6)$ & $99.8(11)$ \\
\hline $\mathrm{C}(14)-\mathrm{C}(15)-\mathrm{C}(16)-\mathrm{C}(12)$ & $30.8(11)$ & $\mathrm{C}(30)-\mathrm{C}(31)-\mathrm{C}(32)-\mathrm{N}(5)$ & $163.0(9)$ \\
\hline $\mathrm{N}(2)-\mathrm{C}(12)-\mathrm{C}(16)-\mathrm{C}(15)$ & $-130.3(9)$ & $\mathrm{C}(27)-\mathrm{C}(31)-\mathrm{C}(32)-\mathrm{N}(5)$ & $-78.8(11)$ \\
\hline$C(13)-C(12)-C(16)-C(15)$ & $-7.6(10)$ & $\mathrm{C}(32)-\mathrm{N}(5)-\mathrm{C}(33)-\mathrm{C}(37)$ & $-92.2(11)$ \\
\hline $\mathrm{N}(2)-\mathrm{C}(12)-\mathrm{C}(16)-\mathrm{C}(17)$ & $105.8(9)$ & $\mathrm{C}(32)-\mathrm{N}(5)-\mathrm{C}(33)-\mathrm{C}(34)$ & 149.1(9) \\
\hline $\mathrm{C}(13)-\mathrm{C}(12)-\mathrm{C}(16)-\mathrm{C}(17)$ & $-131.4(9)$ & N(5)-C(33)-C(34)-C(35) & $121.9(9)$ \\
\hline C(18)-N(3)-C(17)-O(4) & $6.0(15)$ & $\mathrm{C}(37)-\mathrm{C}(33)-\mathrm{C}(34)-\mathrm{C}(35)$ & $-0.6(11)$ \\
\hline $\mathrm{C}(18)-\mathrm{N}(3)-\mathrm{C}(17)-\mathrm{C}(16)$ & $-174.3(8)$ & $\mathrm{C}(33)-\mathrm{C}(34)-\mathrm{C}(35)-\mathrm{C}(36)$ & $27.7(12)$ \\
\hline $\mathrm{C}(15)-\mathrm{C}(16)-\mathrm{C}(17)-\mathrm{O}(4)$ & $-68.0(13)$ & $C(34)-C(35)-C(36)-C(37)$ & $-44.0(12)$ \\
\hline $\mathrm{C}(12)-\mathrm{C}(16)-\mathrm{C}(17)-\mathrm{O}(4)$ & $49.8(12)$ & $\mathrm{C}(35)-\mathrm{C}(36)-\mathrm{C}(37)-\mathrm{C}(38)$ & $161.5(10)$ \\
\hline$C(15)-C(16)-C(17)-N(3)$ & $112.3(10)$ & $\mathrm{C}(35)-\mathrm{C}(36)-\mathrm{C}(37)-\mathrm{C}(33)$ & $42.3(11)$ \\
\hline $\mathrm{C}(12)-\mathrm{C}(16)-\mathrm{C}(17)-\mathrm{N}(3)$ & $-129.8(9)$ & $\mathrm{N}(5)-\mathrm{C}(33)-\mathrm{C}(37)-\mathrm{C}(38)$ & $90.1(10)$ \\
\hline $\mathrm{C}(17)-\mathrm{N}(3)-\mathrm{C}(18)-\mathrm{C}(26)$ & $-69.9(11)$ & $\mathrm{C}(34)-\mathrm{C}(33)-\mathrm{C}(37)-\mathrm{C}(38)$ & $-147.8(9)$ \\
\hline $\mathrm{C}(17)-\mathrm{N}(3)-\mathrm{C}(18)-\mathrm{C}(19)$ & $166.3(8)$ & $\mathrm{N}(5)-\mathrm{C}(33)-\mathrm{C}(37)-\mathrm{C}(36)$ & $-146.0(9)$ \\
\hline N(3)-C(18)-C(19)-C(20) & $-59.7(11)$ & $\mathrm{C}(34)-\mathrm{C}(33)-\mathrm{C}(37)-\mathrm{C}(36)$ & $-24.0(11)$ \\
\hline $\mathrm{C}(26)-\mathrm{C}(18)-\mathrm{C}(19)-\mathrm{C}(20)$ & $172.5(8)$ & $\mathrm{C}(39)-\mathrm{N}(6)-\mathrm{C}(38)-\mathrm{O}(7)$ & $-11.6(14)$ \\
\hline
\end{tabular}




\begin{tabular}{|c|c|c|c|}
\hline $\mathrm{C}(39)-\mathrm{N}(6)-\mathrm{C}(38)-\mathrm{C}(37)$ & $166.4(9)$ & $\mathrm{C}(44)-\mathrm{C}(45)-\mathrm{C}(46)-\mathrm{C}(41)$ & $4(2)$ \\
\hline $\mathrm{C}(36)-\mathrm{C}(37)-\mathrm{C}(38)-\mathrm{O}(7)$ & $-35.3(13)$ & $\mathrm{C}(48)-\mathrm{O}(9)-\mathrm{C}(47)-\mathrm{O}(8)$ & $-0.9(14)$ \\
\hline $\mathrm{C}(33)-\mathrm{C}(37)-\mathrm{C}(38)-\mathrm{O}(7)$ & $79.3(11)$ & $\mathrm{C}(48)-\mathrm{O}(9)-\mathrm{C}(47)-\mathrm{C}(39)$ & $178.5(9)$ \\
\hline $\mathrm{C}(36)-\mathrm{C}(37)-\mathrm{C}(38)-\mathrm{N}(6)$ & $146.7(9)$ & $\mathrm{N}(6)-\mathrm{C}(39)-\mathrm{C}(47)-\mathrm{O}(8)$ & $-50.2(13)$ \\
\hline $\mathrm{C}(33)-\mathrm{C}(37)-\mathrm{C}(38)-\mathrm{N}(6)$ & $-98.7(10)$ & $\mathrm{C}(40)-\mathrm{C}(39)-\mathrm{C}(47)-\mathrm{O}(8)$ & $75.6(13)$ \\
\hline $\mathrm{C}(38)-\mathrm{N}(6)-\mathrm{C}(39)-\mathrm{C}(47)$ & $-131.3(9)$ & N(6)-C(39)-C(47)-O(9) & $130.5(9)$ \\
\hline $\mathrm{C}(38)-\mathrm{N}(6)-\mathrm{C}(39)-\mathrm{C}(40)$ & $105.7(10)$ & $\mathrm{C}(40)-\mathrm{C}(39)-\mathrm{C}(47)-\mathrm{O}(9)$ & $-103.7(10)$ \\
\hline $\mathrm{N}(6)-\mathrm{C}(39)-\mathrm{C}(40)-\mathrm{C}(41)$ & $-173.7(9)$ & $\mathrm{C}(47)-\mathrm{O}(9)-\mathrm{C}(48)-\mathrm{C}(49)$ & $168.3(9)$ \\
\hline $\mathrm{C}(47)-\mathrm{C}(39)-\mathrm{C}(40)-\mathrm{C}(41)$ & $62.8(12)$ & $\mathrm{O}(9)-\mathrm{C}(48)-\mathrm{C}(49)-\mathrm{C}(50)$ & $-83.0(14)$ \\
\hline $\mathrm{C}(39)-\mathrm{C}(40)-\mathrm{C}(41)-\mathrm{C}(46)$ & $77.4(14)$ & $\mathrm{O}(9)-\mathrm{C}(48)-\mathrm{C}(49)-\mathrm{C}(54)$ & $92.9(12)$ \\
\hline $\mathrm{C}(39)-\mathrm{C}(40)-\mathrm{C}(41)-\mathrm{C}(42)$ & $-96.3(14)$ & $\mathrm{C}(54)-\mathrm{C}(49)-\mathrm{C}(50)-\mathrm{C}(51)$ & $-2.5(18)$ \\
\hline$C(46)-C(41)-C(42)-C(43)$ & $-1(2)$ & $\mathrm{C}(48)-\mathrm{C}(49)-\mathrm{C}(50)-\mathrm{C}(51)$ & $173.3(11)$ \\
\hline$C(40)-C(41)-C(42)-C(43)$ & $173.2(14)$ & $\mathrm{C}(49)-\mathrm{C}(50)-\mathrm{C}(51)-\mathrm{C}(52)$ & $3.2(19)$ \\
\hline$C(41)-C(42)-C(43)-C(44)$ & $-1(3)$ & $\mathrm{C}(50)-\mathrm{C}(51)-\mathrm{C}(52)-\mathrm{C}(53)$ & $-4.4(18)$ \\
\hline$C(42)-C(43)-C(44)-C(45)$ & $3(3)$ & $\mathrm{C}(51)-\mathrm{C}(52)-\mathrm{C}(53)-\mathrm{C}(54)$ & $5.4(19)$ \\
\hline$C(43)-C(44)-C(45)-C(46)$ & $-5(3)$ & $\mathrm{C}(52)-\mathrm{C}(53)-\mathrm{C}(54)-\mathrm{C}(49)$ & $-4.8(18)$ \\
\hline $\mathrm{C}(42)-\mathrm{C}(41)-\mathrm{C}(46)-\mathrm{C}(45)$ & $-1(2)$ & $\mathrm{C}(50)-\mathrm{C}(49)-\mathrm{C}(54)-\mathrm{C}(53)$ & 3.3(17) \\
\hline $\mathrm{C}(40)-\mathrm{C}(41)-\mathrm{C}(46)-\mathrm{C}(45)$ & $-174.5(12)$ & $\mathrm{C}(48)-\mathrm{C}(49)-\mathrm{C}(54)-\mathrm{C}(53)$ & $-172.8(11)$ \\
\hline
\end{tabular}

Symmetry transformations used to generate equivalent atoms:

Table S3. Hydrogen bonds for 3 [ $\AA$ and ${ }^{\circ}$.

\begin{tabular}{lcccc}
\hline D-H...A & d(D-H) & d(H...A & $d($ D ...A $)$ & $<($ DHA $)$ \\
\hline $\mathrm{N}(1)-\mathrm{H}(1) \ldots \mathrm{O}(7) \# 1$ & 0.88 & 2.01 & $2.857(10)$ & 160.3 \\
$\mathrm{~N}(2)-\mathrm{H}(2) \ldots \mathrm{O}(6) \# 1$ & 0.88 & 2.07 & $2.928(11)$ & 165.7 \\
$\mathrm{~N}(3)-\mathrm{H}(3) \ldots \mathrm{O}(2)$ & 0.88 & 2.07 & $2.940(11)$ & 168.3 \\
$\mathrm{~N}(4)-\mathrm{H}(4) \ldots \mathrm{O}(3)$ & 0.88 & 2.17 & $2.929(10)$ & 143.7 \\
$\mathrm{~N}(5)-\mathrm{H}(5) \ldots \mathrm{O}(4)$ & 0.88 & 2.31 & $3.006(11)$ & 136.6 \\
$\mathrm{~N}(6)-\mathrm{H}(6) \ldots \mathrm{O}(5)$ & 0.88 & 2.07 & $2.935(11)$ & 167.4 \\
\end{tabular}

Symmetry transformations used to generate equivalent atoms:

$\# 1-\mathrm{y}+1, \mathrm{x}-\mathrm{y}, \mathrm{z}-1 / 3$ 


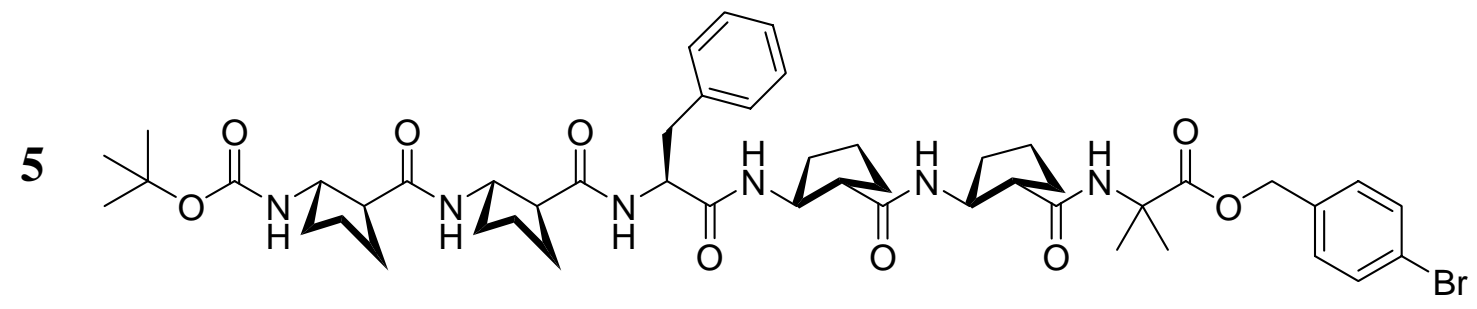

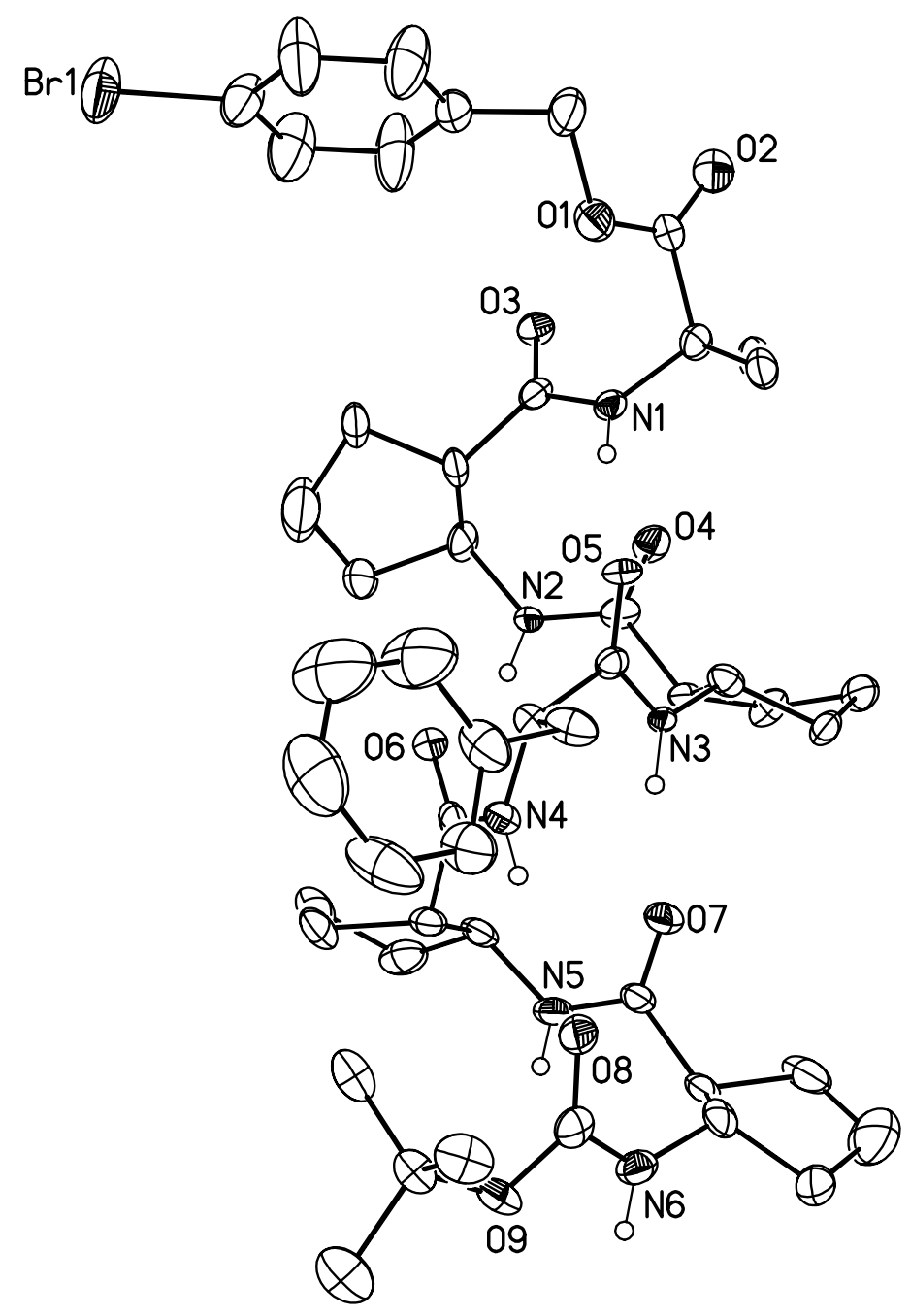

Figure S3. Solid state conformation of $\mathbf{5}$. The molecular diagram is drawn with $30 \%$ probability ellipsoids. Details will be published in the future. 
<smiles>CC(C)(C)OC(=O)NC1CC2CC1C2C(=O)NC1CC2CC(C(=O)NC(C)(C)C(=O)NC3CC4CC3CC4NC(=O)C(C)(C)C(=O)OCc3ccccc3)C1C2</smiles>

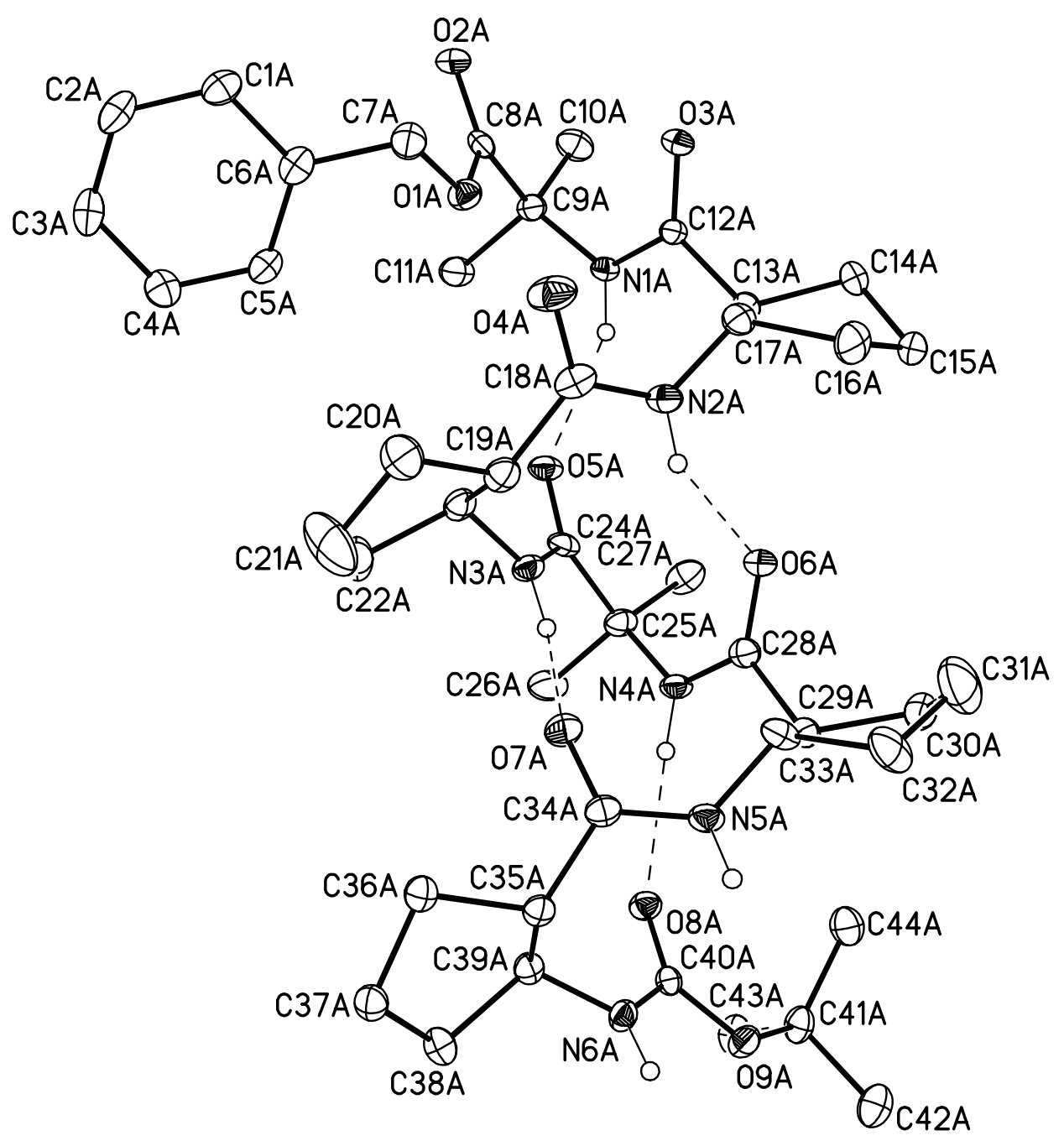

Figure S4. Solid state conformation of 6 . The molecular diagram is drawn with $40 \%$ probability ellipsoids. Details will be published in the future. 


\section{Data Collection for 4}

A colorless crystal with approximate dimensions $0.37 \times 0.25 \times 0.12 \mathrm{~mm}^{3}$ was selected under oil under ambient conditions and attached to the tip of a nylon loop. The crystal was mounted in a stream of cold nitrogen at 100(2) K and centered in the X-ray beam by using a video camera.

The crystal evaluation and data collection were performed on a Bruker CCD-1000 diffractometer with $\mathrm{Mo} \mathrm{K}_{\alpha} \quad(\lambda=0.71073 \AA)$ radiation and the diffractometer to crystal distance of $4.9 \mathrm{~cm}$.

The initial cell constants were obtained from three series of $\omega$ scans at different starting angles. Each series consisted of 20 frames collected at intervals of $0.3^{\circ}$ in a $6^{\circ}$ range about $\omega$ with the exposure time of 10 seconds per frame. A total of 67 reflections was obtained. The reflections were successfully indexed by an automated indexing routine built in the SMART program. The final cell constants were calculated from a set of 17017 strong reflections from the actual data collection.

The data were collected by using the full sphere data collection routine to survey the reciprocal space to the extent of a full sphere to a resolution of $0.87 \AA$. A total of 88255 data were harvested by collecting three sets of frames with $0.25^{\circ}$ scans in $\omega$ with an exposure time $72 \mathrm{sec}$ per frame. These datasets were corrected for Lorentz and polarization effects. The absorption correction was based on fitting a function to the empirical transmission surface as sampled by multiple equivalent measurements. [2]

\section{Structure Solution and Refinement of 4}

The systematic absences in the diffraction data were consistent for the space groups $P \overline{1}$ and $P 1$. The $E$-statistics strongly suggested the non-centrosymmetric space group $P 1$ that yielded chemically reasonable and computationally stable results of refinement [2].

A successful solution by the direct methods provided most non-hydrogen atoms from the E-map. The remaining non-hydrogen atoms were located in an alternating series of least- 
squares cycles and difference Fourier maps. All non-hydrogen atoms were refined with anisotropic displacement coefficients unless otherwise specified. All hydrogen atoms were included in the structure factor calculation at idealized positions and were allowed to ride on the neighboring atoms with relative isotropic displacement coefficients.

There are seven symmetry independent molecules of the peptide in the unit cell. There are also 13.5 molecules of solvated chloroform (that allowed to confirm the absolute configuration) and 0.3 molecules of solvated water. The chloroform molecule containing $\mathrm{C} 7 \mathrm{~s}$ is equally disordered over two positions and was refined isotropically. Chloroform molecule containing $\mathrm{C} 14 \mathrm{~s}$ and solvent water molecule with $\mathrm{O} 1 \mathrm{w}$ were also refined isotropically. The hydrogen atoms on the solvated water molecule were not located.

The final least-squares refinement of 4441 parameters against 55183 data resulted in residuals $R$ (based on $F^{2}$ for $I \geq 2 \sigma$ ) and $w R$ (based on $F^{2}$ for all data) of 0.0643 and 0.1753, respectively. The final difference Fourier map contained a few peaks in the vicinity of solvent molecule with $\mathrm{C} 14 \mathrm{~s}$ and a significant amount of time was invested in refining the disordered molecules but none of the models were computationally stable.

The ORTEP diagram is drawn with $40 \%$ probability ellipsoids.

\section{References}

[1] Lee, H. -S.; LePlae, P. R.; Porter, E. A.; Gellman, S. H. J. Org. Chem. 2001, 66, 3597.

[2] Bruker-AXS. (2000-2003) SADABS V.2.05, SAINT V.6.22, SHELXTL V.6.10

\& SMART 5.622 Software Reference Manuals. Bruker-AXS, Madison, Wisconsin, USA. 


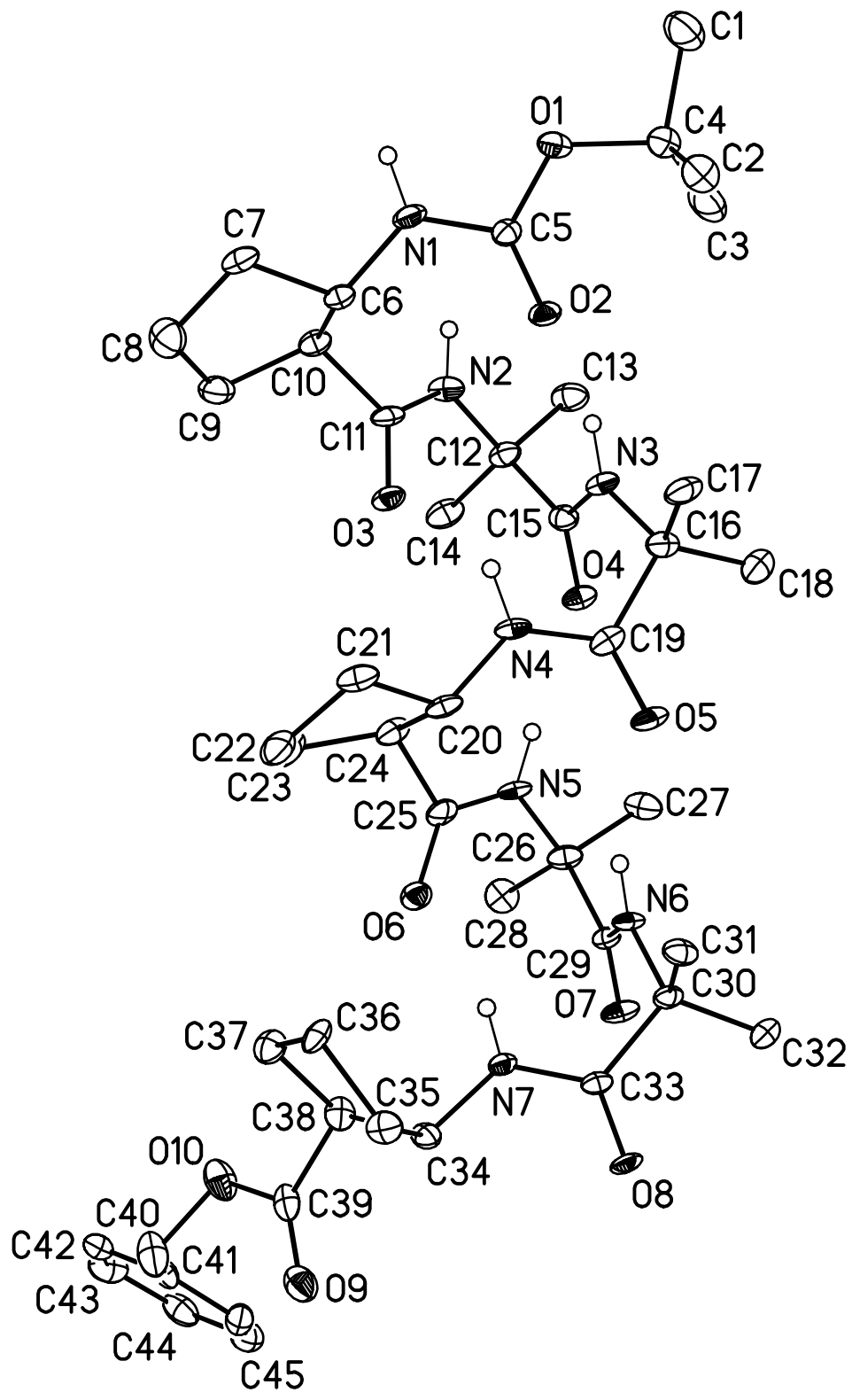

Figure S5. The first symmetry independent molecule of 4. All H atoms except those on the $\mathrm{N}$ atoms are omitted. 


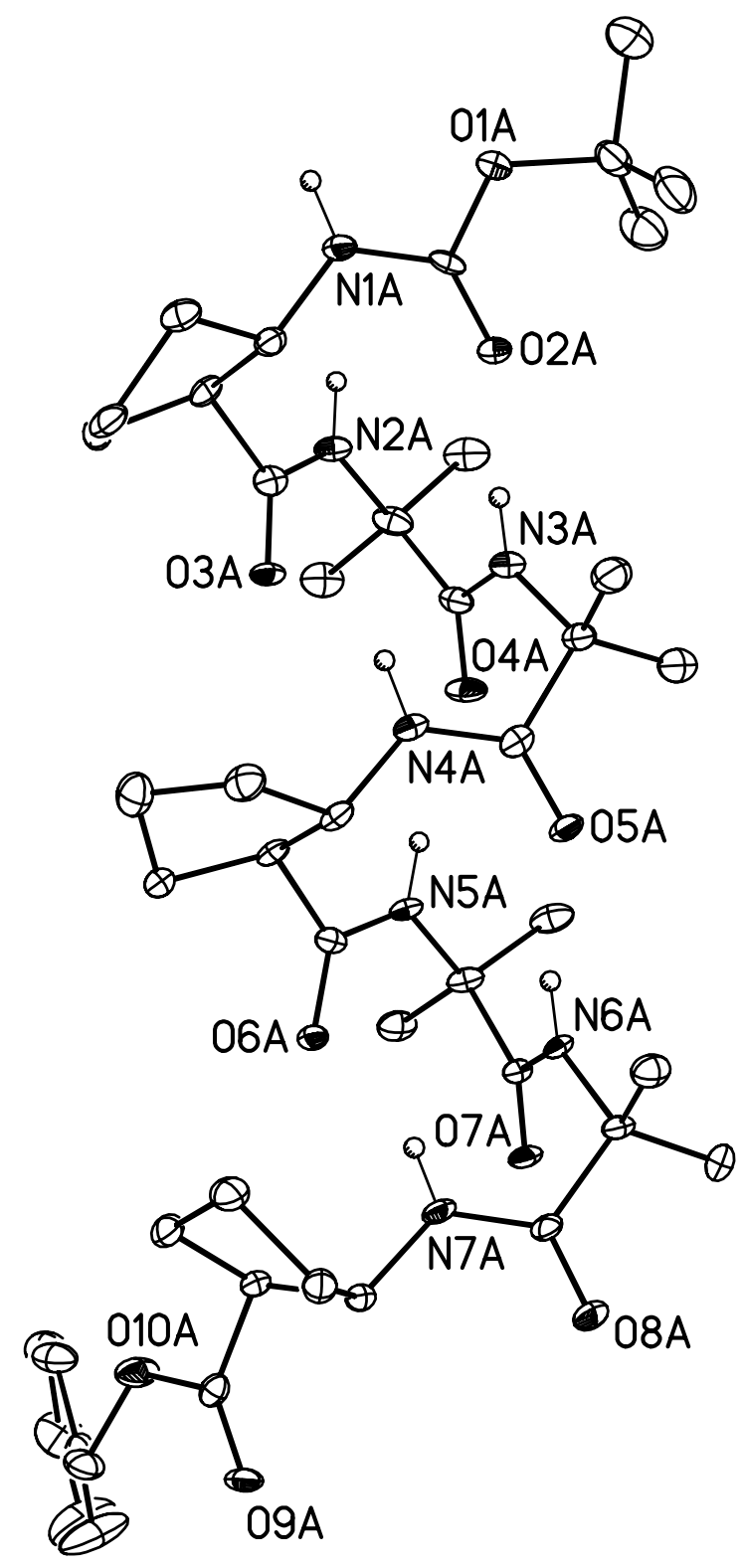

Figure S6. The second symmetry independent molecule of 4 . All $\mathrm{H}$ atoms except those on the $\mathrm{N}$ atoms are omitted. 


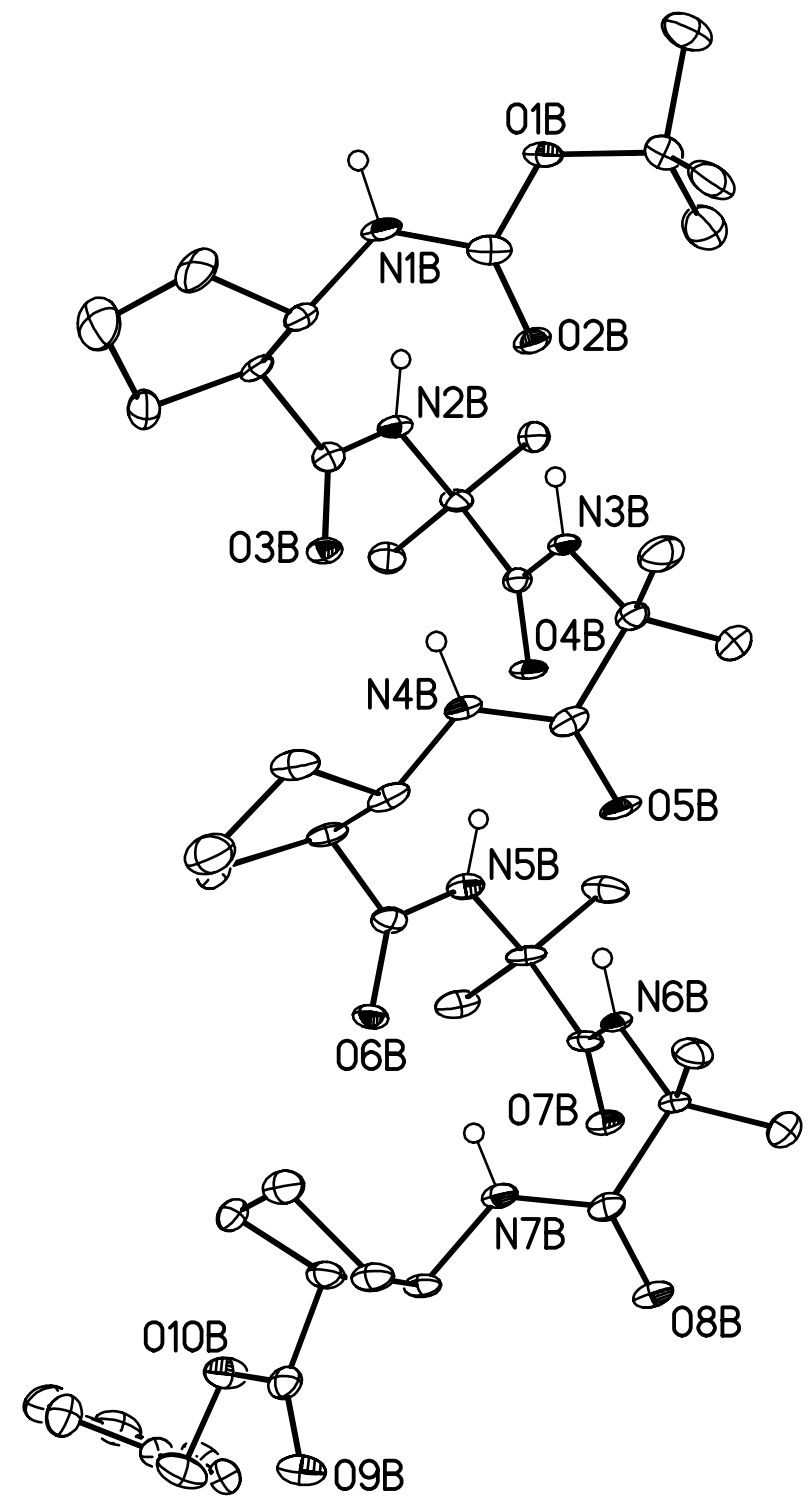

Figure S7. The third symmetry independent molecule of 4 . All $\mathrm{H}$ atoms except those on the $\mathrm{N}$ atoms are omitted. 


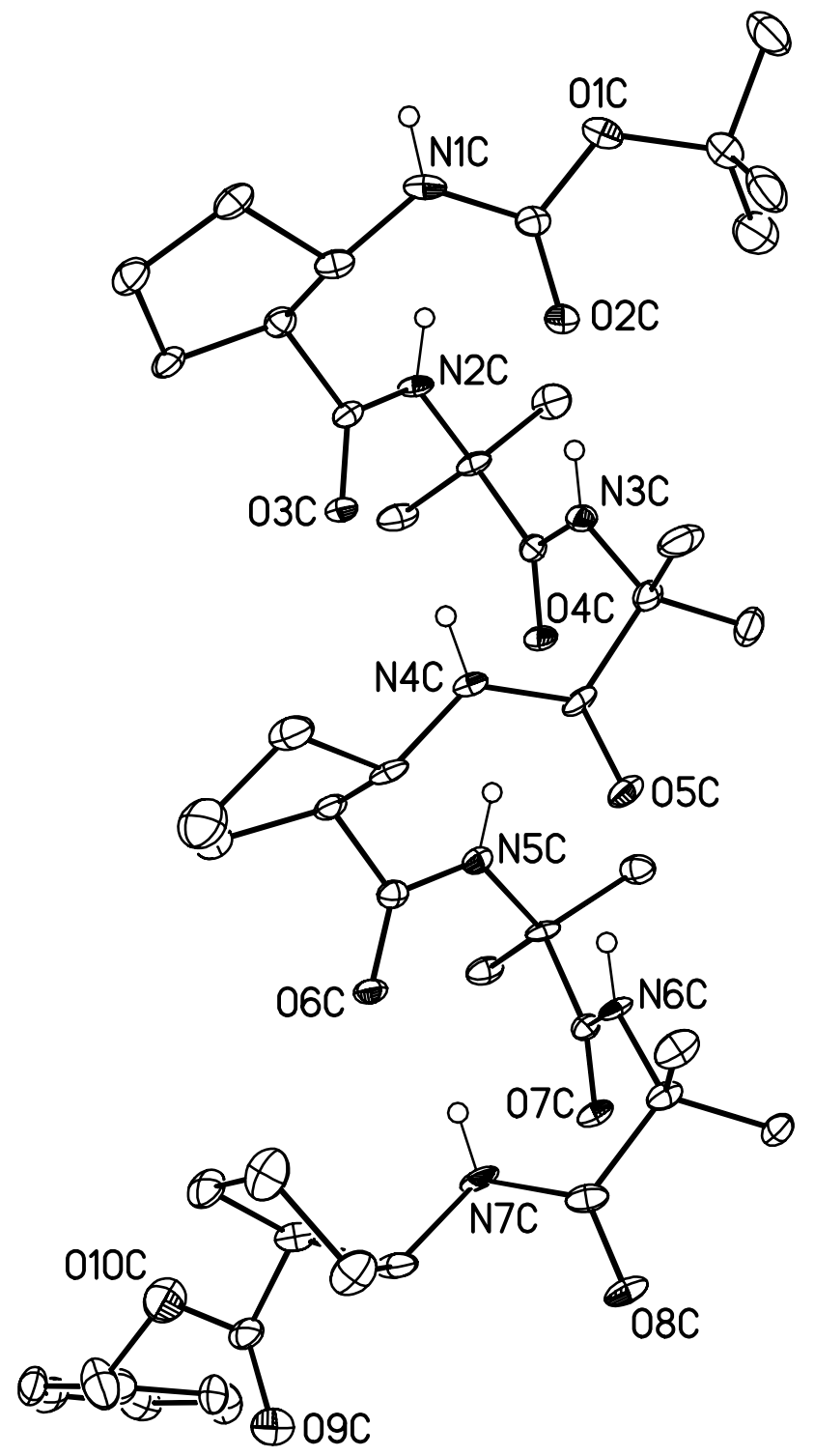

Figure S8. The fourth symmetry independent molecule of 4. All H atoms except those on the $\mathrm{N}$ atoms are omitted. 


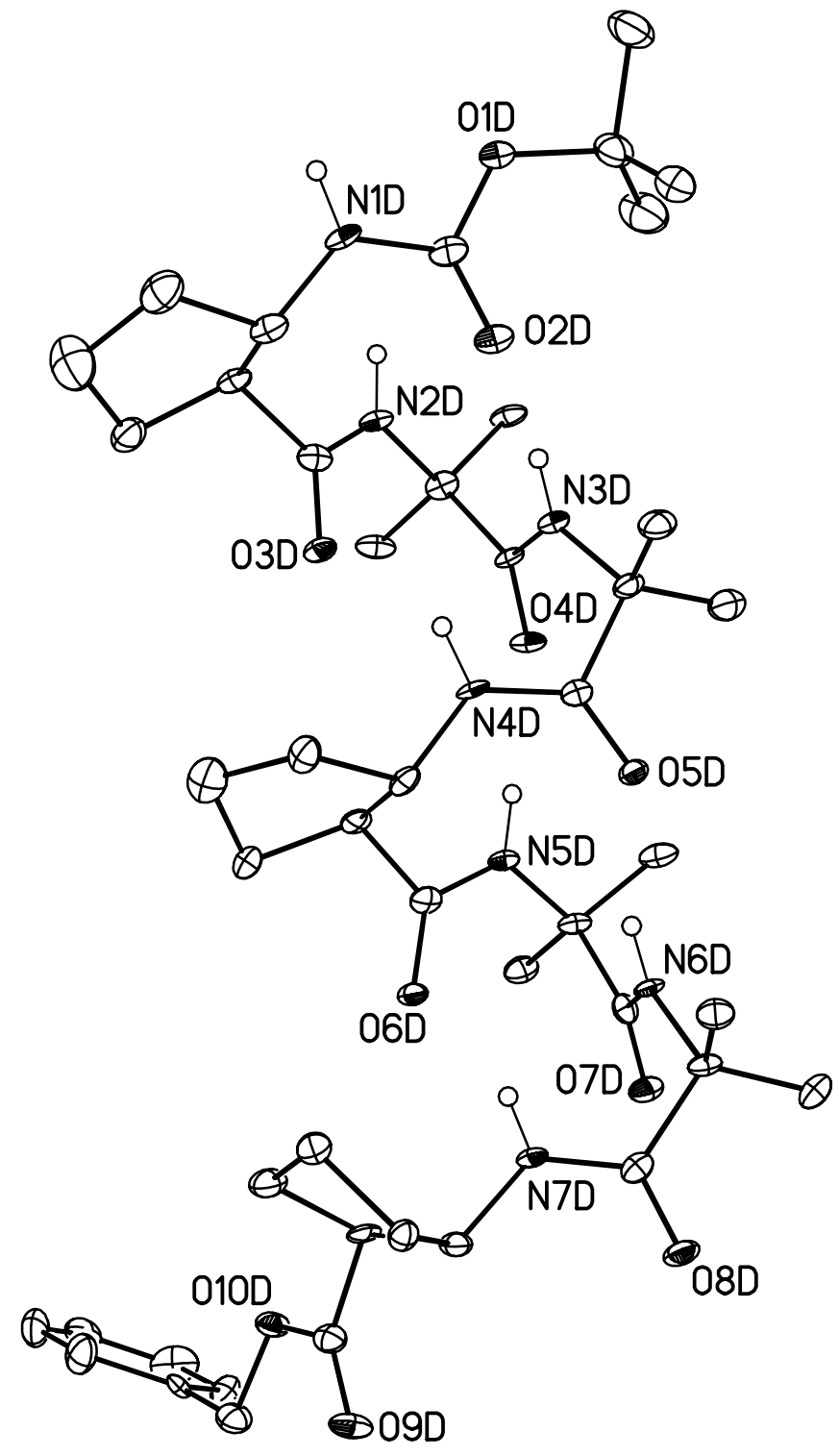

Figure S9. The fifth symmetry independent molecule of 4 . All $\mathrm{H}$ atoms except those on the $\mathrm{N}$ atoms are omitted. 


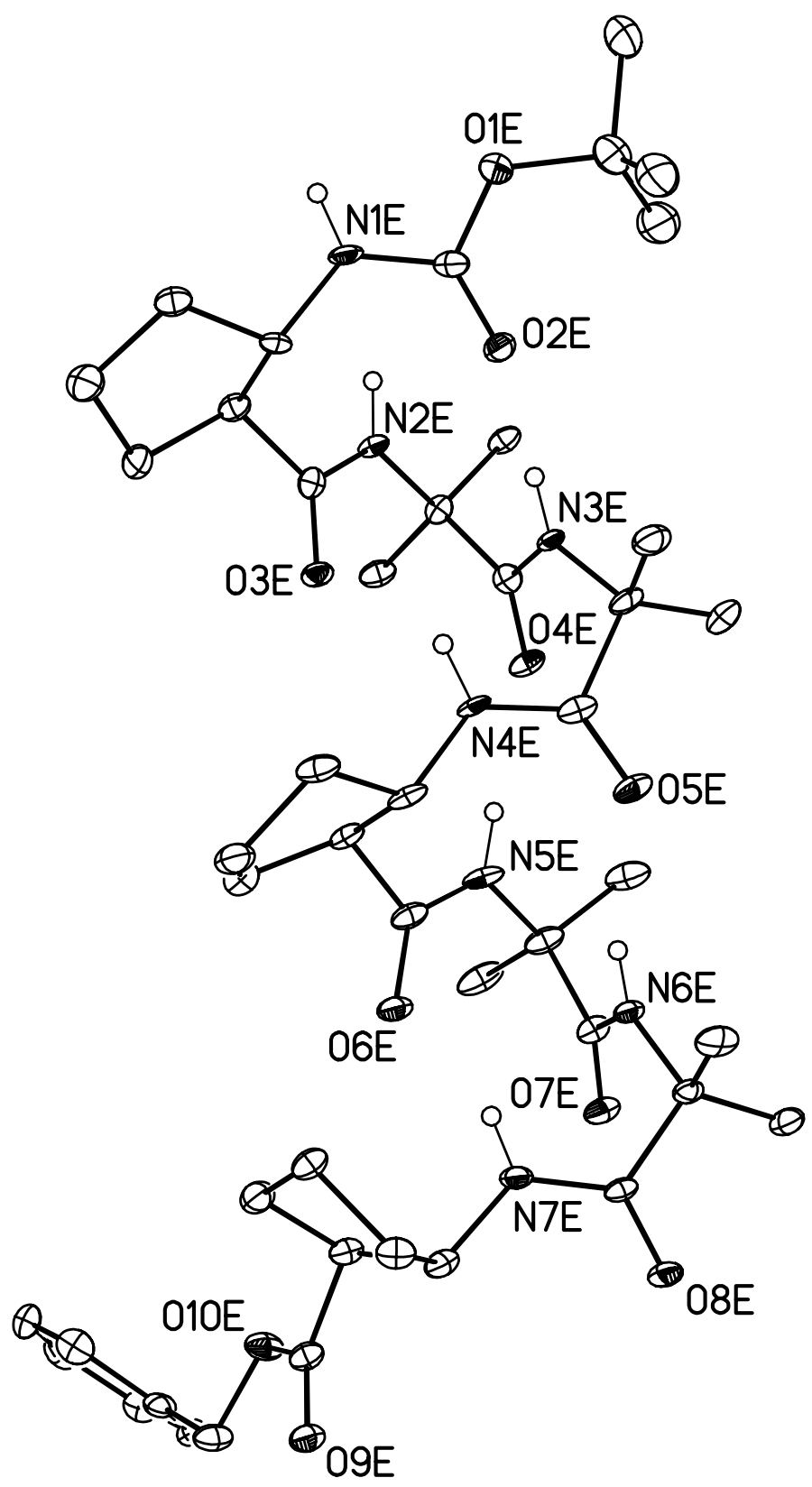

Figure S10. The sixth symmetry independent molecule of 4 . All $\mathrm{H}$ atoms except those on the $\mathrm{N}$ atoms are omitted. 


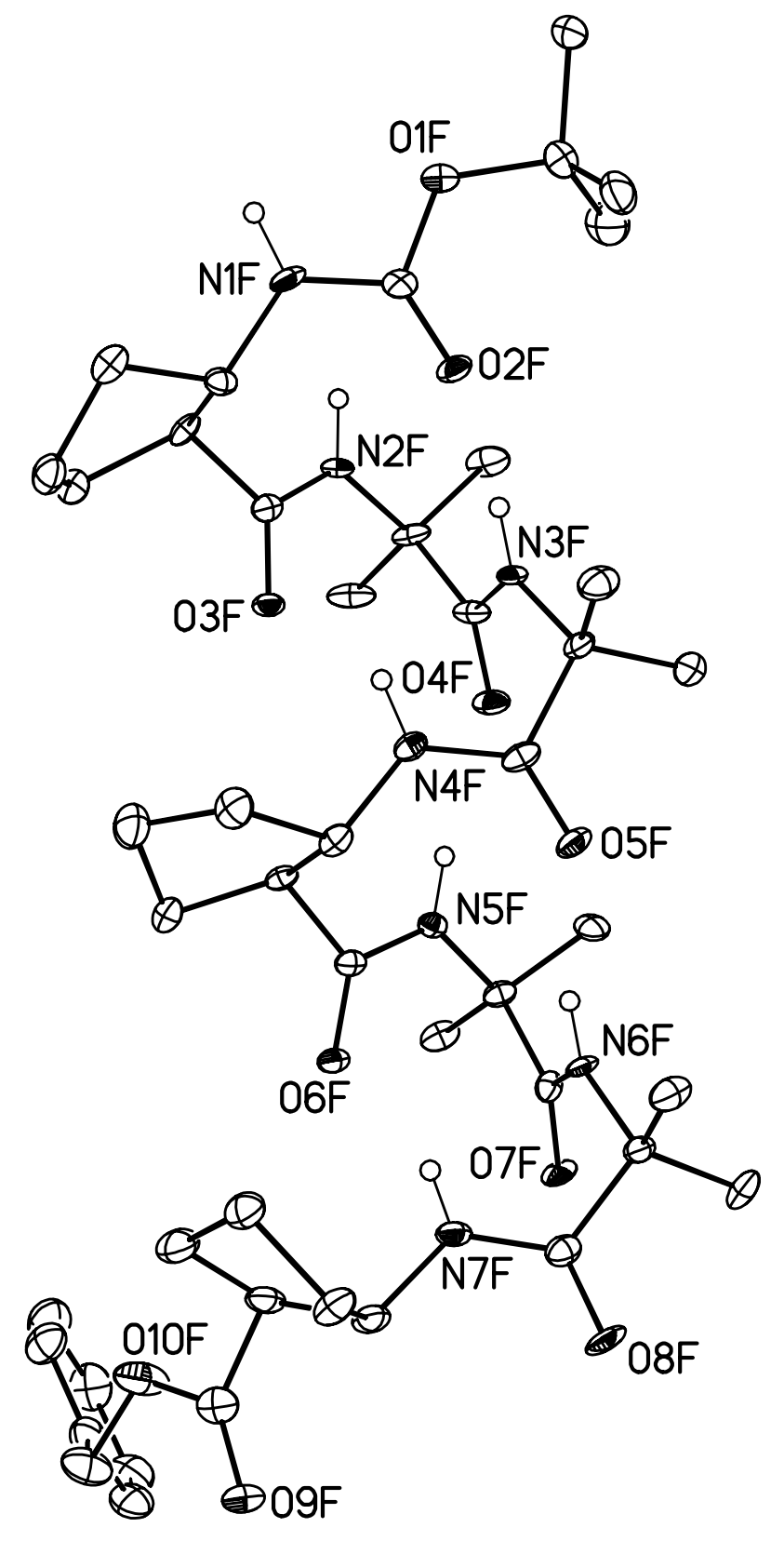

Figure S11. The seventh symmetry independent molecule of 4 . All H atoms except those on the $\mathrm{N}$ atoms are omitted. 


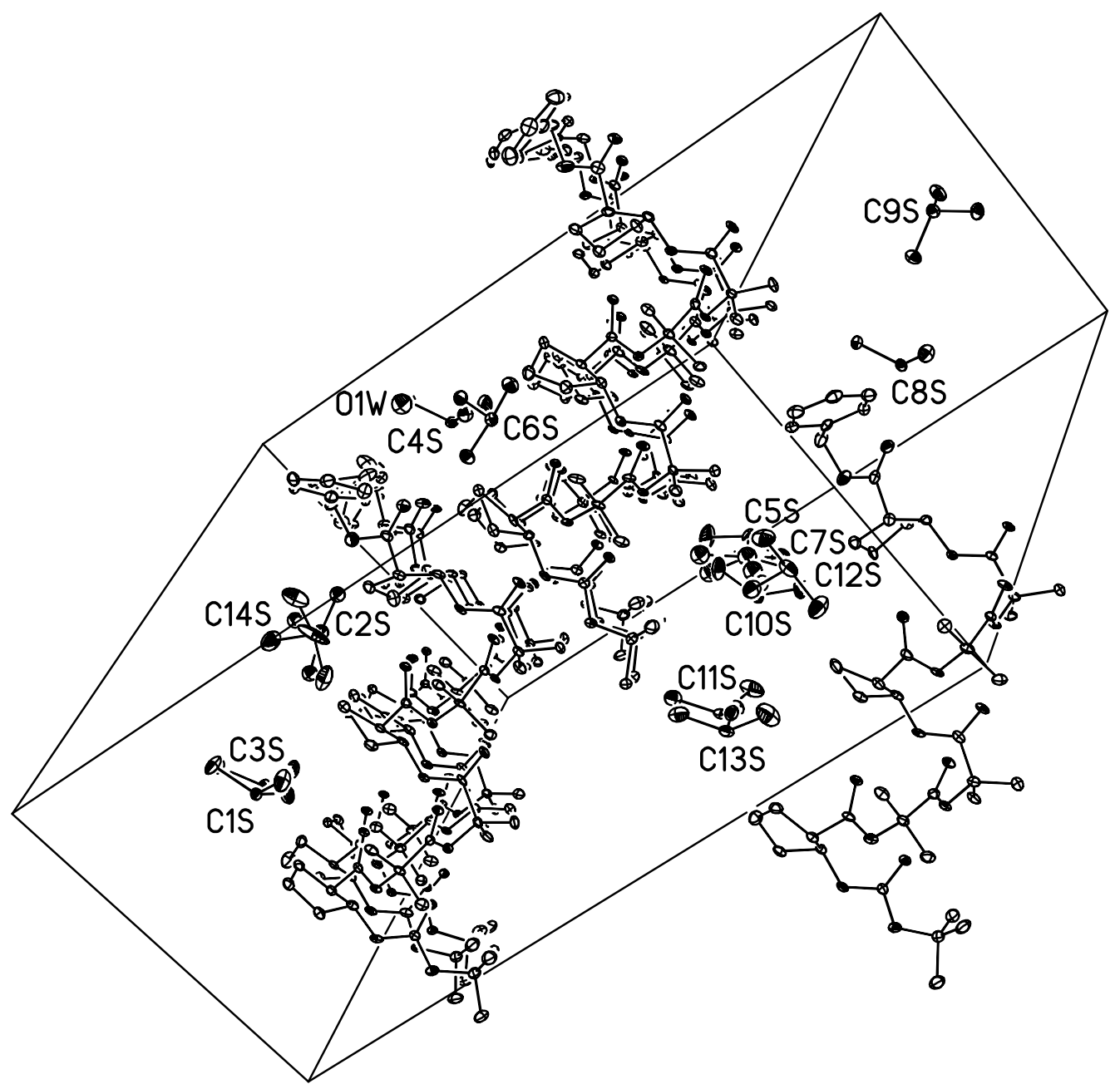

Figure S12. The content of the unit cell of 4. The orientation is along the line of packing of the 6 peptide molecules. Essentially, you see 6 pairwise stacked peptide molecules on the left side and center and the seventh molecule on the right hand side. All the solvent molecules reside between the layers of the peptide molecules and only the $\mathrm{C}$ atoms of the chloroforms and the $\mathrm{O} 1 \mathrm{w}$ atom are labeled. All $\mathrm{H}$ atoms are omitted. 
Table S4. Crystal data and structure refinement for 4.

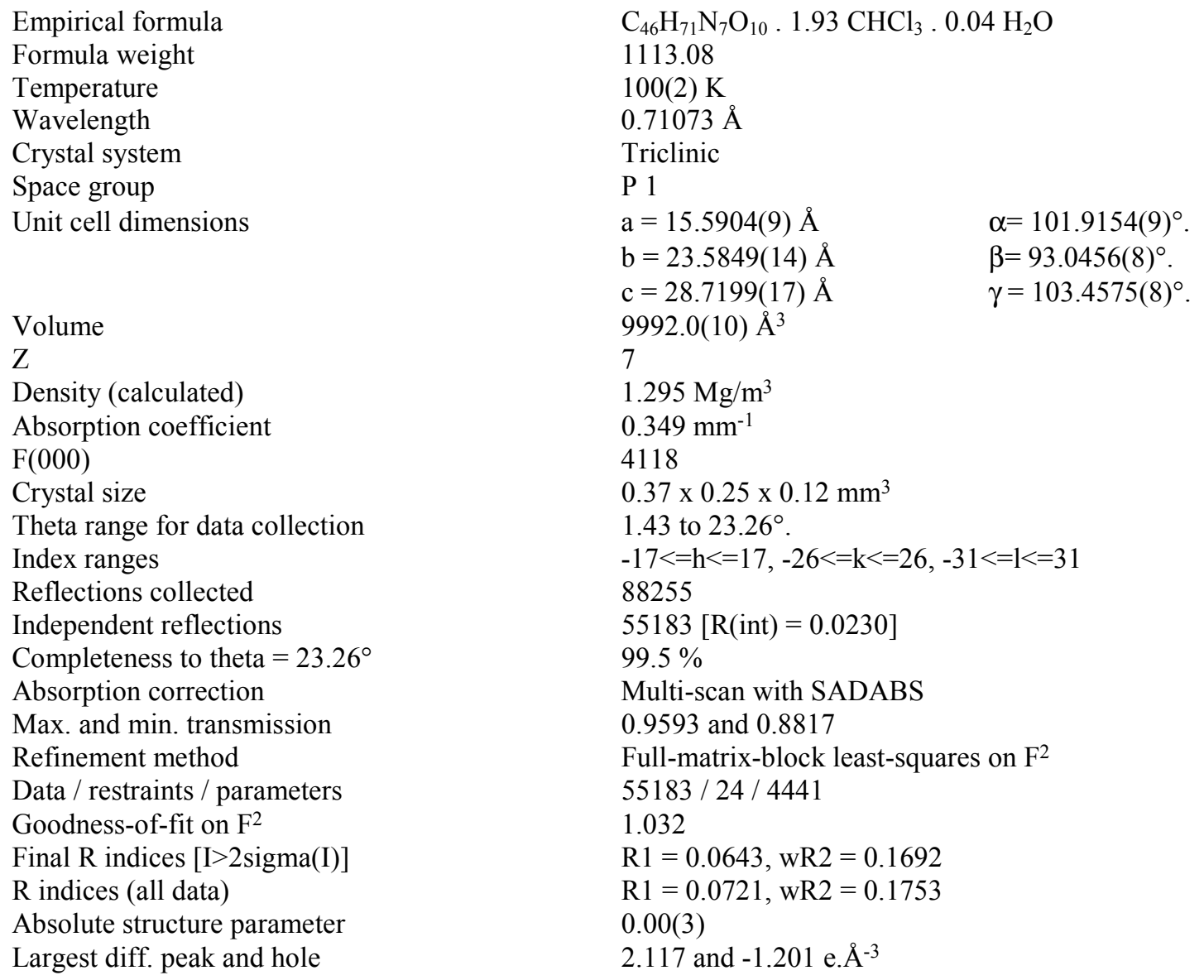


Table S5. Torsion angles $\left[{ }^{\circ}\right]$ for $\mathbf{5}$.

\begin{tabular}{|c|c|c|c|}
\hline $\mathrm{C}(5)-\mathrm{O}(1)-\mathrm{C}(4)-\mathrm{C}(2)$ & $-62.3(6)$ & $\mathrm{C}(22)-\mathrm{C}(23)-\mathrm{C}(24)-\mathrm{C}(25)$ & $115.3(5)$ \\
\hline $\mathrm{C}(5)-\mathrm{O}(1)-\mathrm{C}(4)-\mathrm{C}(3)$ & $64.3(6)$ & $\mathrm{C}(22)-\mathrm{C}(23)-\mathrm{C}(24)-\mathrm{C}(20)$ & $-6.5(5)$ \\
\hline $\mathrm{C}(5)-\mathrm{O}(1)-\mathrm{C}(4)-\mathrm{C}(1)$ & $-179.7(4)$ & $\mathrm{N}(4)-\mathrm{C}(20)-\mathrm{C}(24)-\mathrm{C}(25)$ & $95.8(5)$ \\
\hline $\mathrm{C}(6)-\mathrm{N}(1)-\mathrm{C}(5)-\mathrm{O}(2)$ & $1.4(7)$ & $\mathrm{C}(21)-\mathrm{C}(20)-\mathrm{C}(24)-\mathrm{C}(25)$ & $-142.8(4)$ \\
\hline $\mathrm{C}(6)-\mathrm{N}(1)-\mathrm{C}(5)-\mathrm{O}(1)$ & $-179.7(4)$ & $\mathrm{N}(4)-\mathrm{C}(20)-\mathrm{C}(24)-\mathrm{C}(23)$ & $-139.6(4)$ \\
\hline $\mathrm{C}(4)-\mathrm{O}(1)-\mathrm{C}(5)-\mathrm{O}(2)$ & $-8.5(7)$ & $\mathrm{C}(21)-\mathrm{C}(20)-\mathrm{C}(24)-\mathrm{C}(23)$ & $-18.2(5)$ \\
\hline $\mathrm{C}(4)-\mathrm{O}(1)-\mathrm{C}(5)-\mathrm{N}(1)$ & $172.6(4)$ & $\mathrm{C}(26)-\mathrm{N}(5)-\mathrm{C}(25)-\mathrm{O}(6)$ & $5.2(7)$ \\
\hline $\mathrm{C}(5)-\mathrm{N}(1)-\mathrm{C}(6)-\mathrm{C}(7)$ & $156.0(4)$ & $\mathrm{C}(26)-\mathrm{N}(5)-\mathrm{C}(25)-\mathrm{C}(24)$ & $-177.7(4)$ \\
\hline $\mathrm{C}(5)-\mathrm{N}(1)-\mathrm{C}(6)-\mathrm{C}(10)$ & $-87.0(5)$ & $\mathrm{C}(23)-\mathrm{C}(24)-\mathrm{C}(25)-\mathrm{O}(6)$ & $-27.2(6)$ \\
\hline $\mathrm{N}(1)-\mathrm{C}(6)-\mathrm{C}(7)-\mathrm{C}(8)$ & $159.5(4)$ & $\mathrm{C}(20)-\mathrm{C}(24)-\mathrm{C}(25)-\mathrm{O}(6)$ & $92.0(5)$ \\
\hline$C(10)-C(6)-C(7)-C(8)$ & $36.3(5)$ & $\mathrm{C}(23)-\mathrm{C}(24)-\mathrm{C}(25)-\mathrm{N}(5)$ & $155.7(4)$ \\
\hline $\mathrm{C}(6)-\mathrm{C}(7)-\mathrm{C}(8)-\mathrm{C}(9)$ & $-22.2(6)$ & $\mathrm{C}(20)-\mathrm{C}(24)-\mathrm{C}(25)-\mathrm{N}(5)$ & $-85.1(5)$ \\
\hline $\mathrm{C}(7)-\mathrm{C}(8)-\mathrm{C}(9)-\mathrm{C}(10)$ & $-0.6(6)$ & $\mathrm{C}(25)-\mathrm{N}(5)-\mathrm{C}(26)-\mathrm{C}(27)$ & $-175.3(4)$ \\
\hline $\mathrm{C}(8)-\mathrm{C}(9)-\mathrm{C}(10)-\mathrm{C}(11)$ & $144.1(4)$ & $\mathrm{C}(25)-\mathrm{N}(5)-\mathrm{C}(26)-\mathrm{C}(29)$ & $-57.1(6)$ \\
\hline $\mathrm{C}(8)-\mathrm{C}(9)-\mathrm{C}(10)-\mathrm{C}(6)$ & $22.6(5)$ & $\mathrm{C}(25)-\mathrm{N}(5)-\mathrm{C}(26)-\mathrm{C}(28)$ & $64.7(6)$ \\
\hline $\mathrm{N}(1)-\mathrm{C}(6)-\mathrm{C}(10)-\mathrm{C}(11)$ & $82.6(5)$ & $\mathrm{C}(30)-\mathrm{N}(6)-\mathrm{C}(29)-\mathrm{O}(7)$ & $-3.6(7)$ \\
\hline$C(7)-C(6)-C(10)-C(11)$ & $-156.6(4)$ & $\mathrm{C}(30)-\mathrm{N}(6)-\mathrm{C}(29)-\mathrm{C}(26)$ & $177.8(4)$ \\
\hline $\mathrm{N}(1)-\mathrm{C}(6)-\mathrm{C}(10)-\mathrm{C}(9)$ & $-157.0(4)$ & $\mathrm{N}(5)-\mathrm{C}(26)-\mathrm{C}(29)-\mathrm{O}(7)$ & $154.2(4)$ \\
\hline$C(7)-C(6)-C(10)-C(9)$ & $-36.2(5)$ & $\mathrm{C}(27)-\mathrm{C}(26)-\mathrm{C}(29)-\mathrm{O}(7)$ & $-88.1(5)$ \\
\hline $\mathrm{C}(12)-\mathrm{N}(2)-\mathrm{C}(11)-\mathrm{O}(3)$ & $10.3(7)$ & $\mathrm{C}(28)-\mathrm{C}(26)-\mathrm{C}(29)-\mathrm{O}(7)$ & $32.0(6)$ \\
\hline $\mathrm{C}(12)-\mathrm{N}(2)-\mathrm{C}(11)-\mathrm{C}(10)$ & $-169.1(4)$ & $\mathrm{N}(5)-\mathrm{C}(26)-\mathrm{C}(29)-\mathrm{N}(6)$ & $-27.1(6)$ \\
\hline $\mathrm{C}(9)-\mathrm{C}(10)-\mathrm{C}(11)-\mathrm{O}(3)$ & $-39.1(6)$ & $\mathrm{C}(27)-\mathrm{C}(26)-\mathrm{C}(29)-\mathrm{N}(6)$ & $90.6(5)$ \\
\hline $\mathrm{C}(6)-\mathrm{C}(10)-\mathrm{C}(11)-\mathrm{O}(3)$ & $76.8(6)$ & $\mathrm{C}(28)-\mathrm{C}(26)-\mathrm{C}(29)-\mathrm{N}(6)$ & $-149.4(4)$ \\
\hline $\mathrm{C}(9)-\mathrm{C}(10)-\mathrm{C}(11)-\mathrm{N}(2)$ & $140.4(4)$ & $\mathrm{C}(29)-\mathrm{N}(6)-\mathrm{C}(30)-\mathrm{C}(32)$ & $62.4(6)$ \\
\hline $\mathrm{C}(6)-\mathrm{C}(10)-\mathrm{C}(11)-\mathrm{N}(2)$ & $-103.7(5)$ & $\mathrm{C}(29)-\mathrm{N}(6)-\mathrm{C}(30)-\mathrm{C}(31)$ & $-176.6(4)$ \\
\hline $\mathrm{C}(11)-\mathrm{N}(2)-\mathrm{C}(12)-\mathrm{C}(14)$ & $69.2(6)$ & $\mathrm{C}(29)-\mathrm{N}(6)-\mathrm{C}(30)-\mathrm{C}(33)$ & $-61.9(6)$ \\
\hline $\mathrm{C}(11)-\mathrm{N}(2)-\mathrm{C}(12)-\mathrm{C}(15)$ & $-51.9(6)$ & $\mathrm{C}(34)-\mathrm{N}(7)-\mathrm{C}(33)-\mathrm{O}(8)$ & $2.8(7)$ \\
\hline $\mathrm{C}(11)-\mathrm{N}(2)-\mathrm{C}(12)-\mathrm{C}(13)$ & $-170.7(4)$ & $\mathrm{C}(34)-\mathrm{N}(7)-\mathrm{C}(33)-\mathrm{C}(30)$ & $-171.3(4)$ \\
\hline $\mathrm{C}(16)-\mathrm{N}(3)-\mathrm{C}(15)-\mathrm{O}(4)$ & $-3.8(7)$ & $\mathrm{N}(6)-\mathrm{C}(30)-\mathrm{C}(33)-\mathrm{O}(8)$ & $163.3(4)$ \\
\hline $\mathrm{C}(16)-\mathrm{N}(3)-\mathrm{C}(15)-\mathrm{C}(12)$ & $-180.0(4)$ & $\mathrm{C}(32)-\mathrm{C}(30)-\mathrm{C}(33)-\mathrm{O}(8)$ & $38.3(6)$ \\
\hline $\mathrm{N}(2)-\mathrm{C}(12)-\mathrm{C}(15)-\mathrm{O}(4)$ & $146.3(4)$ & $\mathrm{C}(31)-\mathrm{C}(30)-\mathrm{C}(33)-\mathrm{O}(8)$ & $-81.1(5)$ \\
\hline $\mathrm{C}(14)-\mathrm{C}(12)-\mathrm{C}(15)-\mathrm{O}(4)$ & $25.1(6)$ & $\mathrm{N}(6)-\mathrm{C}(30)-\mathrm{C}(33)-\mathrm{N}(7)$ & $-22.5(6)$ \\
\hline $\mathrm{C}(13)-\mathrm{C}(12)-\mathrm{C}(15)-\mathrm{O}(4)$ & $-95.4(5)$ & $\mathrm{C}(32)-\mathrm{C}(30)-\mathrm{C}(33)-\mathrm{N}(7)$ & $-147.5(4)$ \\
\hline $\mathrm{N}(2)-\mathrm{C}(12)-\mathrm{C}(15)-\mathrm{N}(3)$ & $-37.4(6)$ & $\mathrm{C}(31)-\mathrm{C}(30)-\mathrm{C}(33)-\mathrm{N}(7)$ & $93.1(5)$ \\
\hline $\mathrm{C}(14)-\mathrm{C}(12)-\mathrm{C}(15)-\mathrm{N}(3)$ & $-158.6(4)$ & $\mathrm{C}(33)-\mathrm{N}(7)-\mathrm{C}(34)-\mathrm{C}(38)$ & $-156.8(4)$ \\
\hline $\mathrm{C}(13)-\mathrm{C}(12)-\mathrm{C}(15)-\mathrm{N}(3)$ & $80.9(5)$ & $\mathrm{C}(33)-\mathrm{N}(7)-\mathrm{C}(34)-\mathrm{C}(35)$ & $88.8(5)$ \\
\hline $\mathrm{C}(15)-\mathrm{N}(3)-\mathrm{C}(16)-\mathrm{C}(17)$ & $-169.0(4)$ & $\mathrm{N}(7)-\mathrm{C}(34)-\mathrm{C}(35)-\mathrm{C}(36)$ & $78.2(5)$ \\
\hline $\mathrm{C}(15)-\mathrm{N}(3)-\mathrm{C}(16)-\mathrm{C}(18)$ & $70.4(6)$ & $\mathrm{C}(38)-\mathrm{C}(34)-\mathrm{C}(35)-\mathrm{C}(36)$ & $-40.5(5)$ \\
\hline $\mathrm{C}(15)-\mathrm{N}(3)-\mathrm{C}(16)-\mathrm{C}(19)$ & $-52.1(6)$ & $\mathrm{C}(34)-\mathrm{C}(35)-\mathrm{C}(36)-\mathrm{C}(37)$ & $38.8(5)$ \\
\hline $\mathrm{C}(20)-\mathrm{N}(4)-\mathrm{C}(19)-\mathrm{O}(5)$ & $-9.3(7)$ & $\mathrm{C}(35)-\mathrm{C}(36)-\mathrm{C}(37)-\mathrm{C}(38)$ & $-22.3(5)$ \\
\hline $\mathrm{C}(20)-\mathrm{N}(4)-\mathrm{C}(19)-\mathrm{C}(16)$ & $175.1(4)$ & $\mathrm{N}(7)-\mathrm{C}(34)-\mathrm{C}(38)-\mathrm{C}(39)$ & $149.6(4)$ \\
\hline $\mathrm{N}(3)-\mathrm{C}(16)-\mathrm{C}(19)-\mathrm{O}(5)$ & $143.9(4)$ & $\mathrm{C}(35)-\mathrm{C}(34)-\mathrm{C}(38)-\mathrm{C}(39)$ & $-91.8(5)$ \\
\hline $\mathrm{C}(17)-\mathrm{C}(16)-\mathrm{C}(19)-\mathrm{O}(5)$ & $-98.2(5)$ & $\mathrm{N}(7)-\mathrm{C}(34)-\mathrm{C}(38)-\mathrm{C}(37)$ & $-91.8(5)$ \\
\hline $\mathrm{C}(18)-\mathrm{C}(16)-\mathrm{C}(19)-\mathrm{O}(5)$ & $21.2(6)$ & $\mathrm{C}(35)-\mathrm{C}(34)-\mathrm{C}(38)-\mathrm{C}(37)$ & $26.8(5)$ \\
\hline $\mathrm{N}(3)-\mathrm{C}(16)-\mathrm{C}(19)-\mathrm{N}(4)$ & $-40.4(5)$ & $\mathrm{C}(36)-\mathrm{C}(37)-\mathrm{C}(38)-\mathrm{C}(39)$ & $118.1(5)$ \\
\hline $\mathrm{C}(17)-\mathrm{C}(16)-\mathrm{C}(19)-\mathrm{N}(4)$ & $77.5(5)$ & $\mathrm{C}(36)-\mathrm{C}(37)-\mathrm{C}(38)-\mathrm{C}(34)$ & $-3.1(5)$ \\
\hline $\mathrm{C}(18)-\mathrm{C}(16)-\mathrm{C}(19)-\mathrm{N}(4)$ & $-163.0(4)$ & $\mathrm{C}(40)-\mathrm{O}(10)-\mathrm{C}(39)-\mathrm{O}(9)$ & $6.7(7)$ \\
\hline $\mathrm{C}(19)-\mathrm{N}(4)-\mathrm{C}(20)-\mathrm{C}(21)$ & $149.0(5)$ & $\mathrm{C}(40)-\mathrm{O}(10)-\mathrm{C}(39)-\mathrm{C}(38)$ & $-170.9(4)$ \\
\hline $\mathrm{C}(19)-\mathrm{N}(4)-\mathrm{C}(20)-\mathrm{C}(24)$ & $-95.0(5)$ & $\mathrm{C}(34)-\mathrm{C}(38)-\mathrm{C}(39)-\mathrm{O}(9)$ & $7.5(7)$ \\
\hline $\mathrm{N}(4)-\mathrm{C}(20)-\mathrm{C}(21)-\mathrm{C}(22)$ & $158.1(4)$ & $\mathrm{C}(37)-\mathrm{C}(38)-\mathrm{C}(39)-\mathrm{O}(9)$ & $-109.6(5)$ \\
\hline $\mathrm{C}(24)-\mathrm{C}(20)-\mathrm{C}(21)-\mathrm{C}(22)$ & $36.7(5)$ & $\mathrm{C}(34)-\mathrm{C}(38)-\mathrm{C}(39)-\mathrm{O}(10)$ & $-174.8(4)$ \\
\hline$C(20)-C(21)-C(22)-C(23)$ & $-41.4(5)$ & $\mathrm{C}(37)-\mathrm{C}(38)-\mathrm{C}(39)-\mathrm{O}(10)$ & $68.0(5)$ \\
\hline $\mathrm{C}(21)-\mathrm{C}(22)-\mathrm{C}(23)-\mathrm{C}(24)$ & $29.6(5)$ & $\mathrm{C}(39)-\mathrm{O}(10)-\mathrm{C}(40)-\mathrm{C}(41)$ & $-117.0(5)$ \\
\hline
\end{tabular}


$\mathrm{O}(10)-\mathrm{C}(40)-\mathrm{C}(41)-\mathrm{C}(46)$

$\mathrm{O}(10)-\mathrm{C}(40)-\mathrm{C}(41)-\mathrm{C}(42)$

$\mathrm{C}(46)-\mathrm{C}(41)-\mathrm{C}(42)-\mathrm{C}(43)$

$\mathrm{C}(40)-\mathrm{C}(41)-\mathrm{C}(42)-\mathrm{C}(43)$

$\mathrm{C}(41)-\mathrm{C}(42)-\mathrm{C}(43)-\mathrm{C}(44)$

$\mathrm{C}(42)-\mathrm{C}(43)-\mathrm{C}(44)-\mathrm{C}(45)$

C(43)-C(44)-C(45)-C(46)

$\mathrm{C}(44)-\mathrm{C}(45)-\mathrm{C}(46)-\mathrm{C}(41)$

$\mathrm{C}(42)-\mathrm{C}(41)-\mathrm{C}(46)-\mathrm{C}(45)$

$\mathrm{C}(40)-\mathrm{C}(41)-\mathrm{C}(46)-\mathrm{C}(45)$

$\mathrm{C}(5 \mathrm{~A})-\mathrm{O}(1 \mathrm{~A})-\mathrm{C}(4 \mathrm{~A})-\mathrm{C}(2 \mathrm{~A})$

$\mathrm{C}(5 \mathrm{~A})-\mathrm{O}(1 \mathrm{~A})-\mathrm{C}(4 \mathrm{~A})-\mathrm{C}(1 \mathrm{~A})$

$\mathrm{C}(5 \mathrm{~A})-\mathrm{O}(1 \mathrm{~A})-\mathrm{C}(4 \mathrm{~A})-\mathrm{C}(3 \mathrm{~A})$

$\mathrm{C}(4 \mathrm{~A})-\mathrm{O}(1 \mathrm{~A})-\mathrm{C}(5 \mathrm{~A})-\mathrm{O}(2 \mathrm{~A})$

$\mathrm{C}(4 \mathrm{~A})-\mathrm{O}(1 \mathrm{~A})-\mathrm{C}(5 \mathrm{~A})-\mathrm{N}(1 \mathrm{~A})$

C (6A)-N(1A)-C(5A)-O(2A)

C(6A)-N(1A)-C(5A)-O(1A)

$\mathrm{C}(5 \mathrm{~A})-\mathrm{N}(1 \mathrm{~A})-\mathrm{C}(6 \mathrm{~A})-\mathrm{C}(7 \mathrm{~A})$

$\mathrm{C}(5 \mathrm{~A})-\mathrm{N}(1 \mathrm{~A})-\mathrm{C}(6 \mathrm{~A})-\mathrm{C}(10 \mathrm{~A})$

$\mathrm{N}(1 \mathrm{~A})-\mathrm{C}(6 \mathrm{~A})-\mathrm{C}(7 \mathrm{~A})-\mathrm{C}(8 \mathrm{~A})$

$\mathrm{C}(10 \mathrm{~A})-\mathrm{C}(6 \mathrm{~A})-\mathrm{C}(7 \mathrm{~A})-\mathrm{C}(8 \mathrm{~A})$

$\mathrm{C}(6 \mathrm{~A})-\mathrm{C}(7 \mathrm{~A})-\mathrm{C}(8 \mathrm{~A})-\mathrm{C}(9 \mathrm{~A})$

$\mathrm{C}(7 \mathrm{~A})-\mathrm{C}(8 \mathrm{~A})-\mathrm{C}(9 \mathrm{~A})-\mathrm{C}(10 \mathrm{~A})$

$\mathrm{N}(1 \mathrm{~A})-\mathrm{C}(6 \mathrm{~A})-\mathrm{C}(10 \mathrm{~A})-\mathrm{C}(11 \mathrm{~A})$

$\mathrm{C}(7 \mathrm{~A})-\mathrm{C}(6 \mathrm{~A})-\mathrm{C}(10 \mathrm{~A})-\mathrm{C}(11 \mathrm{~A})$

$\mathrm{N}(1 \mathrm{~A})-\mathrm{C}(6 \mathrm{~A})-\mathrm{C}(10 \mathrm{~A})-\mathrm{C}(9 \mathrm{~A})$

$\mathrm{C}(7 \mathrm{~A})-\mathrm{C}(6 \mathrm{~A})-\mathrm{C}(10 \mathrm{~A})-\mathrm{C}(9 \mathrm{~A})$

$\mathrm{C}(8 \mathrm{~A})-\mathrm{C}(9 \mathrm{~A})-\mathrm{C}(10 \mathrm{~A})-\mathrm{C}(11 \mathrm{~A})$

$\mathrm{C}(8 \mathrm{~A})-\mathrm{C}(9 \mathrm{~A})-\mathrm{C}(10 \mathrm{~A})-\mathrm{C}(6 \mathrm{~A})$

$\mathrm{C}(12 \mathrm{~A})-\mathrm{N}(2 \mathrm{~A})-\mathrm{C}(11 \mathrm{~A})-\mathrm{O}(3 \mathrm{~A})$

$\mathrm{C}(12 \mathrm{~A})-\mathrm{N}(2 \mathrm{~A})-\mathrm{C}(11 \mathrm{~A})-\mathrm{C}(10 \mathrm{~A})$

$\mathrm{C}(6 \mathrm{~A})-\mathrm{C}(10 \mathrm{~A})-\mathrm{C}(11 \mathrm{~A})-\mathrm{O}(3 \mathrm{~A})$

$\mathrm{C}(9 \mathrm{~A})-\mathrm{C}(10 \mathrm{~A})-\mathrm{C}(11 \mathrm{~A})-\mathrm{O}(3 \mathrm{~A})$

$\mathrm{C}(6 \mathrm{~A})-\mathrm{C}(10 \mathrm{~A})-\mathrm{C}(11 \mathrm{~A})-\mathrm{N}(2 \mathrm{~A})$

$\mathrm{C}(9 \mathrm{~A})-\mathrm{C}(10 \mathrm{~A})-\mathrm{C}(11 \mathrm{~A})-\mathrm{N}(2 \mathrm{~A})$

$\mathrm{C}(11 \mathrm{~A})-\mathrm{N}(2 \mathrm{~A})-\mathrm{C}(12 \mathrm{~A})-\mathrm{C}(14 \mathrm{~A})$

$\mathrm{C}(11 \mathrm{~A})-\mathrm{N}(2 \mathrm{~A})-\mathrm{C}(12 \mathrm{~A})-\mathrm{C}(15 \mathrm{~A})$

$\mathrm{C}(11 \mathrm{~A})-\mathrm{N}(2 \mathrm{~A})-\mathrm{C}(12 \mathrm{~A})-\mathrm{C}(13 \mathrm{~A})$

C(16A)-N(3A)-C(15A)-O(4A)

$\mathrm{C}(16 \mathrm{~A})-\mathrm{N}(3 \mathrm{~A})-\mathrm{C}(15 \mathrm{~A})-\mathrm{C}(12 \mathrm{~A})$

$\mathrm{N}(2 \mathrm{~A})-\mathrm{C}(12 \mathrm{~A})-\mathrm{C}(15 \mathrm{~A})-\mathrm{O}(4 \mathrm{~A})$

$\mathrm{C}(14 \mathrm{~A})-\mathrm{C}(12 \mathrm{~A})-\mathrm{C}(15 \mathrm{~A})-\mathrm{O}(4 \mathrm{~A})$

$\mathrm{C}(13 \mathrm{~A})-\mathrm{C}(12 \mathrm{~A})-\mathrm{C}(15 \mathrm{~A})-\mathrm{O}(4 \mathrm{~A})$

$\mathrm{N}(2 \mathrm{~A})-\mathrm{C}(12 \mathrm{~A})-\mathrm{C}(15 \mathrm{~A})-\mathrm{N}(3 \mathrm{~A})$

$\mathrm{C}(14 \mathrm{~A})-\mathrm{C}(12 \mathrm{~A})-\mathrm{C}(15 \mathrm{~A})-\mathrm{N}(3 \mathrm{~A})$

$\mathrm{C}(13 \mathrm{~A})-\mathrm{C}(12 \mathrm{~A})-\mathrm{C}(15 \mathrm{~A})-\mathrm{N}(3 \mathrm{~A})$

C(15A)-N(3A)-C(16A)-C(18A)

C(15A)-N(3A)-C(16A)-C(17A)

$\mathrm{C}(15 \mathrm{~A})-\mathrm{N}(3 \mathrm{~A})-\mathrm{C}(16 \mathrm{~A})-\mathrm{C}(19 \mathrm{~A})$

C(20A)-N(4A)-C(19A)-O(5A)

C(20A)-N(4A)-C(19A)-C(16A)

$\mathrm{N}(3 \mathrm{~A})-\mathrm{C}(16 \mathrm{~A})-\mathrm{C}(19 \mathrm{~A})-\mathrm{O}(5 \mathrm{~A})$

$\mathrm{C}(18 \mathrm{~A})-\mathrm{C}(16 \mathrm{~A})-\mathrm{C}(19 \mathrm{~A})-\mathrm{O}(5 \mathrm{~A})$

$\mathrm{C}(17 \mathrm{~A})-\mathrm{C}(16 \mathrm{~A})-\mathrm{C}(19 \mathrm{~A})-\mathrm{O}(5 \mathrm{~A})$

$\mathrm{N}(3 \mathrm{~A})-\mathrm{C}(16 \mathrm{~A})-\mathrm{C}(19 \mathrm{~A})-\mathrm{N}(4 \mathrm{~A})$
72.5(7)

$-110.8(5)$

$-0.4(7)$

$-177.2(5)$

$-0.8(8)$

$0.9(8)$

$0.4(8)$

$-1.7(8)$

1.7(8)

178.3(5)

$-60.7(6)$

$-177.9(4)$

64.4(6)

$-6.9(7)$

$172.8(4)$

1.2(7)

$-178.5(4)$

$140.8(4)$

$-98.2(5)$

168.7(4)

42.0(5)

$-46.1(5)$

$33.2(5)$

$92.0(5)$

$-142.1(4)$

$-147.4(4)$

$-21.5(5)$

$111.8(4)$

$-7.1(5)$

6.3(7)

$-176.6(4)$

$85.5(5)$

$-29.9(6)$

$-91.6(5)$

153.0(4)

73.0(6)

$-48.1(6)$

$-167.0(4)$

$-0.4(7)$

$-176.9(4)$

$142.6(4)$

20.3(6)

$-99.9(5)$

$-41.0(6)$

$-163.2(4)$

76.5(5)

68.3(6)

$-170.6(4)$

$-54.4(6)$

$-7.7(7)$

178.5(4)

150.1(4)

27.0(6)

$-94.0(5)$

$-35.9(6)$
C(18A)-C(16A)-C(19A)-N(4A)

$\mathrm{C}(17 \mathrm{~A})-\mathrm{C}(16 \mathrm{~A})-\mathrm{C}(19 \mathrm{~A})-\mathrm{N}(4 \mathrm{~A})$

C(19A)-N(4A)-C(20A)-C(24A)

$\mathrm{C}(19 \mathrm{~A})-\mathrm{N}(4 \mathrm{~A})-\mathrm{C}(20 \mathrm{~A})-\mathrm{C}(21 \mathrm{~A})$

$\mathrm{N}(4 \mathrm{~A})-\mathrm{C}(20 \mathrm{~A})-\mathrm{C}(21 \mathrm{~A})-\mathrm{C}(22 \mathrm{~A})$

$\mathrm{C}(24 \mathrm{~A})-\mathrm{C}(20 \mathrm{~A})-\mathrm{C}(21 \mathrm{~A})-\mathrm{C}(22 \mathrm{~A})$

$\mathrm{C}(20 \mathrm{~A})-\mathrm{C}(21 \mathrm{~A})-\mathrm{C}(22 \mathrm{~A})-\mathrm{C}(23 \mathrm{~A})$

$\mathrm{C}(21 \mathrm{~A})-\mathrm{C}(22 \mathrm{~A})-\mathrm{C}(23 \mathrm{~A})-\mathrm{C}(24 \mathrm{~A})$

$\mathrm{C}(22 \mathrm{~A})-\mathrm{C}(23 \mathrm{~A})-\mathrm{C}(24 \mathrm{~A})-\mathrm{C}(25 \mathrm{~A})$

$\mathrm{C}(22 \mathrm{~A})-\mathrm{C}(23 \mathrm{~A})-\mathrm{C}(24 \mathrm{~A})-\mathrm{C}(20 \mathrm{~A})$

$\mathrm{N}(4 \mathrm{~A})-\mathrm{C}(20 \mathrm{~A})-\mathrm{C}(24 \mathrm{~A})-\mathrm{C}(25 \mathrm{~A})$

$\mathrm{C}(21 \mathrm{~A})-\mathrm{C}(20 \mathrm{~A})-\mathrm{C}(24 \mathrm{~A})-\mathrm{C}(25 \mathrm{~A})$

$\mathrm{N}(4 \mathrm{~A})-\mathrm{C}(20 \mathrm{~A})-\mathrm{C}(24 \mathrm{~A})-\mathrm{C}(23 \mathrm{~A})$

$\mathrm{C}(21 \mathrm{~A})-\mathrm{C}(20 \mathrm{~A})-\mathrm{C}(24 \mathrm{~A})-\mathrm{C}(23 \mathrm{~A})$

$\mathrm{C}(26 \mathrm{~A})-\mathrm{N}(5 \mathrm{~A})-\mathrm{C}(25 \mathrm{~A})-\mathrm{O}(6 \mathrm{~A})$

$\mathrm{C}(26 \mathrm{~A})-\mathrm{N}(5 \mathrm{~A})-\mathrm{C}(25 \mathrm{~A})-\mathrm{C}(24 \mathrm{~A})$

$\mathrm{C}(23 \mathrm{~A})-\mathrm{C}(24 \mathrm{~A})-\mathrm{C}(25 \mathrm{~A})-\mathrm{O}(6 \mathrm{~A})$

$\mathrm{C}(20 \mathrm{~A})-\mathrm{C}(24 \mathrm{~A})-\mathrm{C}(25 \mathrm{~A})-\mathrm{O}(6 \mathrm{~A})$

$\mathrm{C}(23 \mathrm{~A})-\mathrm{C}(24 \mathrm{~A})-\mathrm{C}(25 \mathrm{~A})-\mathrm{N}(5 \mathrm{~A})$

$\mathrm{C}(20 \mathrm{~A})-\mathrm{C}(24 \mathrm{~A})-\mathrm{C}(25 \mathrm{~A})-\mathrm{N}(5 \mathrm{~A})$

$\mathrm{C}(25 \mathrm{~A})-\mathrm{N}(5 \mathrm{~A})-\mathrm{C}(26 \mathrm{~A})-\mathrm{C}(28 \mathrm{~A})$

$\mathrm{C}(25 \mathrm{~A})-\mathrm{N}(5 \mathrm{~A})-\mathrm{C}(26 \mathrm{~A})-\mathrm{C}(27 \mathrm{~A})$

$\mathrm{C}(25 \mathrm{~A})-\mathrm{N}(5 \mathrm{~A})-\mathrm{C}(26 \mathrm{~A})-\mathrm{C}(29 \mathrm{~A})$

C(30A)-N(6A)-C(29A)-O(7A)

$\mathrm{C}(30 \mathrm{~A})-\mathrm{N}(6 \mathrm{~A})-\mathrm{C}(29 \mathrm{~A})-\mathrm{C}(26 \mathrm{~A})$

$\mathrm{N}(5 \mathrm{~A})-\mathrm{C}(26 \mathrm{~A})-\mathrm{C}(29 \mathrm{~A})-\mathrm{O}(7 \mathrm{~A})$

$\mathrm{C}(28 \mathrm{~A})-\mathrm{C}(26 \mathrm{~A})-\mathrm{C}(29 \mathrm{~A})-\mathrm{O}(7 \mathrm{~A})$

$\mathrm{C}(27 \mathrm{~A})-\mathrm{C}(26 \mathrm{~A})-\mathrm{C}(29 \mathrm{~A})-\mathrm{O}(7 \mathrm{~A})$

$\mathrm{N}(5 \mathrm{~A})-\mathrm{C}(26 \mathrm{~A})-\mathrm{C}(29 \mathrm{~A})-\mathrm{N}(6 \mathrm{~A})$

$\mathrm{C}(28 \mathrm{~A})-\mathrm{C}(26 \mathrm{~A})-\mathrm{C}(29 \mathrm{~A})-\mathrm{N}(6 \mathrm{~A})$

$\mathrm{C}(27 \mathrm{~A})-\mathrm{C}(26 \mathrm{~A})-\mathrm{C}(29 \mathrm{~A})-\mathrm{N}(6 \mathrm{~A})$

$\mathrm{C}(29 \mathrm{~A})-\mathrm{N}(6 \mathrm{~A})-\mathrm{C}(30 \mathrm{~A})-\mathrm{C}(32 \mathrm{~A})$

$\mathrm{C}(29 \mathrm{~A})-\mathrm{N}(6 \mathrm{~A})-\mathrm{C}(30 \mathrm{~A})-\mathrm{C}(33 \mathrm{~A})$

$\mathrm{C}(29 \mathrm{~A})-\mathrm{N}(6 \mathrm{~A})-\mathrm{C}(30 \mathrm{~A})-\mathrm{C}(31 \mathrm{~A})$

$\mathrm{C}(34 \mathrm{~A})-\mathrm{N}(7 \mathrm{~A})-\mathrm{C}(33 \mathrm{~A})-\mathrm{O}(8 \mathrm{~A})$

$\mathrm{C}(34 \mathrm{~A})-\mathrm{N}(7 \mathrm{~A})-\mathrm{C}(33 \mathrm{~A})-\mathrm{C}(30 \mathrm{~A})$

$\mathrm{N}(6 \mathrm{~A})-\mathrm{C}(30 \mathrm{~A})-\mathrm{C}(33 \mathrm{~A})-\mathrm{O}(8 \mathrm{~A})$

$\mathrm{C}(32 \mathrm{~A})-\mathrm{C}(30 \mathrm{~A})-\mathrm{C}(33 \mathrm{~A})-\mathrm{O}(8 \mathrm{~A})$

$\mathrm{C}(31 \mathrm{~A})-\mathrm{C}(30 \mathrm{~A})-\mathrm{C}(33 \mathrm{~A})-\mathrm{O}(8 \mathrm{~A})$

$\mathrm{N}(6 \mathrm{~A})-\mathrm{C}(30 \mathrm{~A})-\mathrm{C}(33 \mathrm{~A})-\mathrm{N}(7 \mathrm{~A})$

$\mathrm{C}(32 \mathrm{~A})-\mathrm{C}(30 \mathrm{~A})-\mathrm{C}(33 \mathrm{~A})-\mathrm{N}(7 \mathrm{~A})$

$\mathrm{C}(31 \mathrm{~A})-\mathrm{C}(30 \mathrm{~A})-\mathrm{C}(33 \mathrm{~A})-\mathrm{N}(7 \mathrm{~A})$

C(33A)-N(7A)-C(34A)-C(35A)

$\mathrm{C}(33 \mathrm{~A})-\mathrm{N}(7 \mathrm{~A})-\mathrm{C}(34 \mathrm{~A})-\mathrm{C}(38 \mathrm{~A})$

$\mathrm{N}(7 \mathrm{~A})-\mathrm{C}(34 \mathrm{~A})-\mathrm{C}(35 \mathrm{~A})-\mathrm{C}(36 \mathrm{~A})$

$\mathrm{C}(38 \mathrm{~A})-\mathrm{C}(34 \mathrm{~A})-\mathrm{C}(35 \mathrm{~A})-\mathrm{C}(36 \mathrm{~A})$

$\mathrm{C}(34 \mathrm{~A})-\mathrm{C}(35 \mathrm{~A})-\mathrm{C}(36 \mathrm{~A})-\mathrm{C}(37 \mathrm{~A})$

$\mathrm{C}(35 \mathrm{~A})-\mathrm{C}(36 \mathrm{~A})-\mathrm{C}(37 \mathrm{~A})-\mathrm{C}(38 \mathrm{~A})$

$\mathrm{N}(7 \mathrm{~A})-\mathrm{C}(34 \mathrm{~A})-\mathrm{C}(38 \mathrm{~A})-\mathrm{C}(39 \mathrm{~A})$

$\mathrm{C}(35 \mathrm{~A})-\mathrm{C}(34 \mathrm{~A})-\mathrm{C}(38 \mathrm{~A})-\mathrm{C}(39 \mathrm{~A})$

$\mathrm{N}(7 \mathrm{~A})-\mathrm{C}(34 \mathrm{~A})-\mathrm{C}(38 \mathrm{~A})-\mathrm{C}(37 \mathrm{~A})$

$\mathrm{C}(35 \mathrm{~A})-\mathrm{C}(34 \mathrm{~A})-\mathrm{C}(38 \mathrm{~A})-\mathrm{C}(37 \mathrm{~A})$

$\mathrm{C}(36 \mathrm{~A})-\mathrm{C}(37 \mathrm{~A})-\mathrm{C}(38 \mathrm{~A})-\mathrm{C}(39 \mathrm{~A})$

$\mathrm{C}(36 \mathrm{~A})-\mathrm{C}(37 \mathrm{~A})-\mathrm{C}(38 \mathrm{~A})-\mathrm{C}(34 \mathrm{~A})$

$\mathrm{C}(40 \mathrm{~A})-\mathrm{O}(10 \mathrm{~A})-\mathrm{C}(39 \mathrm{~A})-\mathrm{O}(9 \mathrm{~A})$
$-158.9(4)$

$80.1(5)$

$-97.3(5)$

$145.1(4)$

$117.9(5)$

$-4.1(5)$

28.5(5)

$-41.8(5)$

$161.9(4)$

$39.6(5)$

91.3(5)

$-146.3(4)$

$-143.9(4)$

$-21.6(5)$

$5.2(7)$

$-179.0(4)$

$-26.8(6)$

$92.0(5)$

$157.5(4)$

$-83.7(5)$

$69.2(5)$

$-169.4(4)$

$-51.9(6)$

$-0.6(7)$

$-176.6(4)$

145.3(4)

23.1(6)

$-96.8(5)$

$-38.5(6)$

$-160.7(4)$

79.4(5)

64.4(5)

$-60.4(5)$

$-176.4(4)$

$0.3(7)$

$-174.2(4)$

162.1(4)

$36.6(6)$

$-81.5(5)$

$-23.3(5)$

$-148.7(4)$

93.2(5)

96.3(5)

$-149.2(4)$

$80.9(5)$

$-37.6(5)$

44.3(5)

$-33.4(5)$

$137.2(4)$

$-103.5(4)$

$-102.6(4)$

16.7(5)

132.2(4)

$10.3(5)$

1.6(7) 


$\begin{array}{lc} & \\ \text { C(40A)-O(10A)-C(39A)-C(38A) } & -175.4(4) \\ \text { C(34A)-C(38A)-C(39A)-O(9A) } & 11.8(7) \\ \text { C(37A)-C(38A)-C(39A)-O(9A) } & -106.6(5) \\ \text { C(34A)-C(38A)-C(39A)-O(10A) } & -171.3(4) \\ \text { C(37A)-C(38A)-C(39A)-O(10A) } & 70.4(5) \\ \text { C(39A)-O(10A)-C(40A)-C(41A) } & -133.3(5) \\ \text { O(10A)-C(40A)-C(41A)-C(46A) } & 141.5(6) \\ \text { O(10A)-C(40A)-C(41A)-C(42A) } & -42.5(7) \\ \text { C(46A)-C(41A)-C(42A)-C(43A) } & -0.8(9) \\ \text { C(40A)-C(41A)-C(42A)-C(43A) } & -176.8(5) \\ \text { C(41A)-C(42A)-C(43A)-C(44A) } & 1.9(9) \\ \text { C(42A)-C(43A)-C(44A)-C(45A) } & -2.1(11) \\ \text { C(43A)-C(44A)-C(45A)-C(46A) } & 1.3(13) \\ \text { C(42A)-C(41A)-C(46A)-C(45A) } & 0.0(12) \\ \text { C(40A)-C(41A)-C(46A)-C(45A) } & 176.2(7) \\ \text { C(44A)-C(45A)-C(46A)-C(41A) } & -0.3(14) \\ \text { C(5B)-O(1B)-C(4B)-C(2B) } & -62.7(6) \\ \text { C(5B)-O(1B)-C(4B)-C(1B) } & -179.8(4) \\ \text { C(5B)-O(1B)-C(4B)-C(3B) } & 63.3(6) \\ \text { C(6B)-N(1B)-C(5B)-O(2B) } & -1.5(8) \\ \text { C(6B)-N(1B)-C(5B)-O(1B) } & 179.2(4) \\ \text { C(4B)-O(1B)-C(5B)-O(2B) } & -7.8(7) \\ \text { C(4B)-O(1B)-C(5B)-N(1B) } & 171.5(4) \\ \text { C(5B)-N(1B)-C(6B)-C(7B) } & 152.3(5) \\ \text { C(5B)-N(1B)-C(6B)-C(10B) } & -89.5(5) \\ \text { N(1B)-C(6B)-C(7B)-C(8B) } & 139.6(5) \\ \text { C(10B)-C(6B)-C(7B)-C(8B) } & 15.8(6) \\ \text { C(6B)-C(7B)-C(8B)-C(9B) } & 6.5(7) \\ \text { C(7B)-C(8B)-C(9B)-C(10B) } & -26.3(7) \\ \text { C(8B)-C(9B)-C(10B)-C(11B) } & 157.6(4) \\ \text { C(8B)-C(9B)-C(10B)-C(6B) } & 35.5(5) \\ \text { N(1B)-C(6B)-C(10B)-C(11B) } & 85.0(5) \\ \text { C(7B)-C(6B)-C(10B)-C(11B) } & -153.6(4) \\ \text { N(1B)-C(6B)-C(10B)-C(9B) } & -153.1(4) \\ \text { C(7B)-C(6B)-C(10B)-C(9B) } & -31.7(5) \\ \text { C(12B)-N(2B)-C(11B)-O(3B) } & 9.3(7) \\ \text { C(12B)-N(2B)-C(11B)-C(10B) } & -172.0(4) \\ \text { C(9B)-C(10B)-C(11B)-O(3B) } & -35.3(6) \\ \text { C(6B)-C(10B)-C(11B)-O(3B) } & 81.5(6) \\ \text { C(9B)-C(10B)-C(11B)-N(2B) } & 146.0(4) \\ \text { C(6B)-C(10B)-C(11B)-N(2B) } & -97.2(5) \\ \text { C(11B)-N(2B)-C(12B)-C(14B) } & 71.7(5) \\ \text { C(11B)-N(2B)-C(12B)-C(13B) } & -168.1(4) \\ \text { C(11B)-N(2B)-C(12B)-C(15B) } & -49.7(6) \\ \text { C(16B)-N(3B)-C(15B)-O(4B) } & -1.9(7) \\ \text { C(16B)-N(3B)-C(15B)-C(12B) } & -177.1(4) \\ \text { N(2B)-C(12B)-C(15B)-O(4B) } & 144.6(4) \\ \text { C(14B)-C(12B)-C(15B)-O(4B) } & 22.7(6) \\ \text { C(13B)-C(12B)-C(15B)-O(4B) } & -97.6(5) \\ \text { N(2B)-C(12B)-C(15B)-N(3B) } & -40.2(5) \\ \text { C(14B)-C(12B)-C(15B)-N(3B) } & -162.1(4) \\ \text { C(13B)-C(12B)-C(15B)-N(3B) } & 77.6(5) \\ \text { C(15B)-N(3B)-C(16B)-C(18B) } & 69.1(6) \\ \text { C(15B)-N(3B)-C(16B)-C(17B) } & -170.5(4) \\ \text { C(15B)-N(3B)-C(16B)-C(19B) } & -53.7(6) \\ & \\ & \end{array}$

C(20B)-N(4B)-C(19B)-O(5B)

C(20B)-N(4B)-C(19B)-C(16B)

$\mathrm{N}(3 \mathrm{~B})-\mathrm{C}(16 \mathrm{~B})-\mathrm{C}(19 \mathrm{~B})-\mathrm{O}(5 \mathrm{~B})$

C(18B)-C(16B)-C(19B)-O(5B)

C(17B)-C(16B)-C(19B)-O(5B)

$\mathrm{N}(3 \mathrm{~B})-\mathrm{C}(16 \mathrm{~B})-\mathrm{C}(19 \mathrm{~B})-\mathrm{N}(4 \mathrm{~B})$

C(18B)-C(16B)-C(19B)-N(4B)

C(17B)-C(16B)-C(19B)-N(4B)

C(19B)-N(4B)-C(20B)-C(21B)

C(19B)-N(4B)-C(20B)-C(24B)

$\mathrm{N}(4 \mathrm{~B})-\mathrm{C}(20 \mathrm{~B})-\mathrm{C}(21 \mathrm{~B})-\mathrm{C}(22 \mathrm{~B})$

$\mathrm{C}(24 \mathrm{~B})-\mathrm{C}(20 \mathrm{~B})-\mathrm{C}(21 \mathrm{~B})-\mathrm{C}(22 \mathrm{~B})$

C(20B)-C(21B)-C(22B)-C(23B)

$\mathrm{C}(21 \mathrm{~B})-\mathrm{C}(22 \mathrm{~B})-\mathrm{C}(23 \mathrm{~B})-\mathrm{C}(24 \mathrm{~B})$

$\mathrm{C}(22 \mathrm{~B})-\mathrm{C}(23 \mathrm{~B})-\mathrm{C}(24 \mathrm{~B})-\mathrm{C}(25 \mathrm{~B})$

$\mathrm{C}(22 \mathrm{~B})-\mathrm{C}(23 \mathrm{~B})-\mathrm{C}(24 \mathrm{~B})-\mathrm{C}(20 \mathrm{~B})$

$\mathrm{N}(4 \mathrm{~B})-\mathrm{C}(20 \mathrm{~B})-\mathrm{C}(24 \mathrm{~B})-\mathrm{C}(25 \mathrm{~B})$

$\mathrm{C}(21 \mathrm{~B})-\mathrm{C}(20 \mathrm{~B})-\mathrm{C}(24 \mathrm{~B})-\mathrm{C}(25 \mathrm{~B})$

$\mathrm{N}(4 \mathrm{~B})-\mathrm{C}(20 \mathrm{~B})-\mathrm{C}(24 \mathrm{~B})-\mathrm{C}(23 \mathrm{~B})$

$\mathrm{C}(21 \mathrm{~B})-\mathrm{C}(20 \mathrm{~B})-\mathrm{C}(24 \mathrm{~B})-\mathrm{C}(23 \mathrm{~B})$

$\mathrm{C}(26 \mathrm{~B})-\mathrm{N}(5 \mathrm{~B})-\mathrm{C}(25 \mathrm{~B})-\mathrm{O}(6 \mathrm{~B})$

$\mathrm{C}(26 \mathrm{~B})-\mathrm{N}(5 \mathrm{~B})-\mathrm{C}(25 \mathrm{~B})-\mathrm{C}(24 \mathrm{~B})$

$\mathrm{C}(23 \mathrm{~B})-\mathrm{C}(24 \mathrm{~B})-\mathrm{C}(25 \mathrm{~B})-\mathrm{O}(6 \mathrm{~B})$

C(20B)-C(24B)-C(25B)-O(6B)

C(23B)-C(24B)-C(25B)-N(5B)

C(20B)-C(24B)-C(25B)-N(5B)

C (25B)-N(5B)-C(26B)-C(28B)

C (25B)-N(5B)-C(26B)-C(27B)

C (25B)-N(5B)-C(26B)-C(29B)

C(30B)-N(6B)-C(29B)-O(7B)

$\mathrm{C}(30 \mathrm{~B})-\mathrm{N}(6 \mathrm{~B})-\mathrm{C}(29 \mathrm{~B})-\mathrm{C}(26 \mathrm{~B})$

$\mathrm{N}(5 \mathrm{~B})-\mathrm{C}(26 \mathrm{~B})-\mathrm{C}(29 \mathrm{~B})-\mathrm{O}(7 \mathrm{~B})$

$\mathrm{C}(28 \mathrm{~B})-\mathrm{C}(26 \mathrm{~B})-\mathrm{C}(29 \mathrm{~B})-\mathrm{O}(7 \mathrm{~B})$

$\mathrm{C}(27 \mathrm{~B})-\mathrm{C}(26 \mathrm{~B})-\mathrm{C}(29 \mathrm{~B})-\mathrm{O}(7 \mathrm{~B})$

$\mathrm{N}(5 \mathrm{~B})-\mathrm{C}(26 \mathrm{~B})-\mathrm{C}(29 \mathrm{~B})-\mathrm{N}(6 \mathrm{~B})$

C (28B)-C(26B)-C(29B)-N(6B)

C(27B)-C(26B)-C(29B)-N(6B)

C(29B)-N(6B)-C(30B)-C(31B)

C(29B)-N(6B)-C(30B)-C(32B)

C(29B)-N(6B)-C(30B)-C(33B)

C(34B)-N(7B)-C(33B)-O(8B)

C(34B)-N(7B)-C(33B)-C(30B)

$\mathrm{N}(6 \mathrm{~B})-\mathrm{C}(30 \mathrm{~B})-\mathrm{C}(33 \mathrm{~B})-\mathrm{O}(8 \mathrm{~B})$

$\mathrm{C}(31 \mathrm{~B})-\mathrm{C}(30 \mathrm{~B})-\mathrm{C}(33 \mathrm{~B})-\mathrm{O}(8 \mathrm{~B})$

$\mathrm{C}(32 \mathrm{~B})-\mathrm{C}(30 \mathrm{~B})-\mathrm{C}(33 \mathrm{~B})-\mathrm{O}(8 \mathrm{~B})$

$\mathrm{N}(6 \mathrm{~B})-\mathrm{C}(30 \mathrm{~B})-\mathrm{C}(33 \mathrm{~B})-\mathrm{N}(7 \mathrm{~B})$

$\mathrm{C}(31 \mathrm{~B})-\mathrm{C}(30 \mathrm{~B})-\mathrm{C}(33 \mathrm{~B})-\mathrm{N}(7 \mathrm{~B})$

C(32B)-C(30B)-C(33B)-N(7B)

C (33B)-N(7B)-C(34B)-C(38B)

C(33B)-N(7B)-C(34B)-C(35B)

$\mathrm{N}(7 \mathrm{~B})-\mathrm{C}(34 \mathrm{~B})-\mathrm{C}(35 \mathrm{~B})-\mathrm{C}(36 \mathrm{~B})$

$\mathrm{C}(38 \mathrm{~B})-\mathrm{C}(34 \mathrm{~B})-\mathrm{C}(35 \mathrm{~B})-\mathrm{C}(36 \mathrm{~B})$

C(34B)-C(35B)-C(36B)-C(37B)

$\mathrm{C}(35 \mathrm{~B})-\mathrm{C}(36 \mathrm{~B})-\mathrm{C}(37 \mathrm{~B})-\mathrm{C}(38 \mathrm{~B})$

N(7B)-C(34B)-C(38B)-C(39B)
$-5.9(7)$

$178.9(4)$

$150.2(4)$

28.1(6)

$-92.8(5)$

$-34.2(6)$

$-156.4(4)$

$82.7(5)$

$142.7(5)$

$-100.4(5)$

163.3(4)

$40.9(5)$

$-41.1(5)$

$25.5(6)$

121.1(5)

$-0.6(5)$

$89.8(5)$

$-148.1(4)$

$-146.1(4)$

$-24.0(5)$

$5.9(7)$

$-177.3(4)$

$-24.1(6)$

94.4(5)

159.1(4)

$-82.4(5)$

$65.0(6)$

$-175.6(4)$

$-58.0(6)$

$-3.3(7)$

178.1(4)

152.9(4)

29.6(6)

$-89.5(5)$

$-28.4(6)$

$-151.7(4)$

89.1(5)

$-176.0(4)$

63.5(6)

$-60.3(6)$

2.9(7)

$-171.5(4)$

161.3(4)

$-81.8(5)$

$37.3(6)$

$-24.1(6)$

92.9(5)

$-148.1(4)$

$-149.2(4)$

96.0(5)

$79.5(5)$

$-38.8(5)$

41.2(5)

$-27.3(5)$

144.5(4) 


\begin{tabular}{|c|c|c|c|}
\hline $\mathrm{C}(35 \mathrm{~B})-\mathrm{C}(34 \mathrm{~B})-\mathrm{C}(38 \mathrm{~B})-\mathrm{C}(39 \mathrm{~B})$ & $-96.8(4)$ & $\mathrm{N}(2 \mathrm{C})-\mathrm{C}(12 \mathrm{C})-\mathrm{C}(15 \mathrm{C})-\mathrm{N}(3 \mathrm{C})$ & $-37.2(6)$ \\
\hline N(7B)-C(34B)-C(38B)-C(37B) & $-97.1(4)$ & $\mathrm{C}(13 \mathrm{C})-\mathrm{C}(12 \mathrm{C})-\mathrm{C}(15 \mathrm{C})-\mathrm{N}(3 \mathrm{C})$ & $81.1(5)$ \\
\hline $\mathrm{C}(35 \mathrm{~B})-\mathrm{C}(34 \mathrm{~B})-\mathrm{C}(38 \mathrm{~B})-\mathrm{C}(37 \mathrm{~B})$ & $21.7(5)$ & $\mathrm{C}(14 \mathrm{C})-\mathrm{C}(12 \mathrm{C})-\mathrm{C}(15 \mathrm{C})-\mathrm{N}(3 \mathrm{C})$ & $-158.8(4)$ \\
\hline $\mathrm{C}(36 \mathrm{~B})-\mathrm{C}(37 \mathrm{~B})-\mathrm{C}(38 \mathrm{~B})-\mathrm{C}(39 \mathrm{~B})$ & $123.6(4)$ & $\mathrm{C}(15 \mathrm{C})-\mathrm{N}(3 \mathrm{C})-\mathrm{C}(16 \mathrm{C})-\mathrm{C}(18 \mathrm{C})$ & $67.1(5)$ \\
\hline $\mathrm{C}(36 \mathrm{~B})-\mathrm{C}(37 \mathrm{~B})-\mathrm{C}(38 \mathrm{~B})-\mathrm{C}(34 \mathrm{~B})$ & $3.3(5)$ & $\mathrm{C}(15 \mathrm{C})-\mathrm{N}(3 \mathrm{C})-\mathrm{C}(16 \mathrm{C})-\mathrm{C}(17 \mathrm{C})$ & $-171.3(4)$ \\
\hline $\mathrm{C}(40 \mathrm{~B})-\mathrm{O}(10 \mathrm{~B})-\mathrm{C}(39 \mathrm{~B})-\mathrm{O}(9 \mathrm{~B})$ & $0.6(7)$ & $\mathrm{C}(15 \mathrm{C})-\mathrm{N}(3 \mathrm{C})-\mathrm{C}(16 \mathrm{C})-\mathrm{C}(19 \mathrm{C})$ & $-55.4(6)$ \\
\hline $\mathrm{C}(40 \mathrm{~B})-\mathrm{O}(10 \mathrm{~B})-\mathrm{C}(39 \mathrm{~B})-\mathrm{C}(38 \mathrm{~B})$ & $-178.3(4)$ & $\mathrm{C}(20 \mathrm{C})-\mathrm{N}(4 \mathrm{C})-\mathrm{C}(19 \mathrm{C})-\mathrm{O}(5 \mathrm{C})$ & $-5.2(7)$ \\
\hline $\mathrm{C}(34 \mathrm{~B})-\mathrm{C}(38 \mathrm{~B})-\mathrm{C}(39 \mathrm{~B})-\mathrm{O}(9 \mathrm{~B})$ & 19.1(7) & $\mathrm{C}(20 \mathrm{C})-\mathrm{N}(4 \mathrm{C})-\mathrm{C}(19 \mathrm{C})-\mathrm{C}(16 \mathrm{C})$ & $179.3(4)$ \\
\hline $\mathrm{C}(37 \mathrm{~B})-\mathrm{C}(38 \mathrm{~B})-\mathrm{C}(39 \mathrm{~B})-\mathrm{O}(9 \mathrm{~B})$ & $-97.7(6)$ & $\mathrm{N}(3 \mathrm{C})-\mathrm{C}(16 \mathrm{C})-\mathrm{C}(19 \mathrm{C})-\mathrm{O}(5 \mathrm{C})$ & $153.0(4)$ \\
\hline $\mathrm{C}(34 \mathrm{~B})-\mathrm{C}(38 \mathrm{~B})-\mathrm{C}(39 \mathrm{~B})-\mathrm{O}(10 \mathrm{~B})$ & $-162.1(4)$ & $\mathrm{C}(18 \mathrm{C})-\mathrm{C}(16 \mathrm{C})-\mathrm{C}(19 \mathrm{C})-\mathrm{O}(5 \mathrm{C})$ & $30.6(6)$ \\
\hline $\mathrm{C}(37 \mathrm{~B})-\mathrm{C}(38 \mathrm{~B})-\mathrm{C}(39 \mathrm{~B})-\mathrm{O}(10 \mathrm{~B})$ & $81.1(5)$ & $\mathrm{C}(17 \mathrm{C})-\mathrm{C}(16 \mathrm{C})-\mathrm{C}(19 \mathrm{C})-\mathrm{O}(5 \mathrm{C})$ & $-89.2(5)$ \\
\hline $\mathrm{C}(39 \mathrm{~B})-\mathrm{O}(10 \mathrm{~B})-\mathrm{C}(40 \mathrm{~B})-\mathrm{C}(41 \mathrm{~B})$ & $-160.3(4)$ & $\mathrm{N}(3 \mathrm{C})-\mathrm{C}(16 \mathrm{C})-\mathrm{C}(19 \mathrm{C})-\mathrm{N}(4 \mathrm{C})$ & $-31.3(5)$ \\
\hline $\mathrm{O}(10 \mathrm{~B})-\mathrm{C}(40 \mathrm{~B})-\mathrm{C}(41 \mathrm{~B})-\mathrm{C}(46 \mathrm{~B})$ & $94.2(6)$ & $\mathrm{C}(18 \mathrm{C})-\mathrm{C}(16 \mathrm{C})-\mathrm{C}(19 \mathrm{C})-\mathrm{N}(4 \mathrm{C})$ & $-153.7(4)$ \\
\hline $\mathrm{O}(10 \mathrm{~B})-\mathrm{C}(40 \mathrm{~B})-\mathrm{C}(41 \mathrm{~B})-\mathrm{C}(42 \mathrm{~B})$ & $-88.6(7)$ & $\mathrm{C}(17 \mathrm{C})-\mathrm{C}(16 \mathrm{C})-\mathrm{C}(19 \mathrm{C})-\mathrm{N}(4 \mathrm{C})$ & $86.5(5)$ \\
\hline $\mathrm{C}(46 \mathrm{~B})-\mathrm{C}(41 \mathrm{~B})-\mathrm{C}(42 \mathrm{~B})-\mathrm{C}(43 \mathrm{~B})$ & $-1.1(9)$ & $\mathrm{C}(19 \mathrm{C})-\mathrm{N}(4 \mathrm{C})-\mathrm{C}(20 \mathrm{C})-\mathrm{C}(21 \mathrm{C})$ & $143.8(4)$ \\
\hline $\mathrm{C}(40 \mathrm{~B})-\mathrm{C}(41 \mathrm{~B})-\mathrm{C}(42 \mathrm{~B})-\mathrm{C}(43 \mathrm{~B})$ & $-178.3(5)$ & $\mathrm{C}(19 \mathrm{C})-\mathrm{N}(4 \mathrm{C})-\mathrm{C}(20 \mathrm{C})-\mathrm{C}(24 \mathrm{C})$ & $-99.6(5)$ \\
\hline $\mathrm{C}(41 \mathrm{~B})-\mathrm{C}(42 \mathrm{~B})-\mathrm{C}(43 \mathrm{~B})-\mathrm{C}(44 \mathrm{~B})$ & $0.6(9)$ & $\mathrm{N}(4 \mathrm{C})-\mathrm{C}(20 \mathrm{C})-\mathrm{C}(21 \mathrm{C})-\mathrm{C}(22 \mathrm{C})$ & $163.2(4)$ \\
\hline $\mathrm{C}(42 \mathrm{~B})-\mathrm{C}(43 \mathrm{~B})-\mathrm{C}(44 \mathrm{~B})-\mathrm{C}(45 \mathrm{~B})$ & $-0.2(9)$ & $\mathrm{C}(24 \mathrm{C})-\mathrm{C}(20 \mathrm{C})-\mathrm{C}(21 \mathrm{C})-\mathrm{C}(22 \mathrm{C})$ & $41.7(5)$ \\
\hline $\mathrm{C}(43 \mathrm{~B})-\mathrm{C}(44 \mathrm{~B})-\mathrm{C}(45 \mathrm{~B})-\mathrm{C}(46 \mathrm{~B})$ & $0.5(9)$ & $C(20 C)-C(21 C)-C(22 C)-C(23 C)$ & $-40.8(6)$ \\
\hline $\mathrm{C}(42 \mathrm{~B})-\mathrm{C}(41 \mathrm{~B})-\mathrm{C}(46 \mathrm{~B})-\mathrm{C}(45 \mathrm{~B})$ & $1.4(8)$ & $\mathrm{C}(21 \mathrm{C})-\mathrm{C}(22 \mathrm{C})-\mathrm{C}(23 \mathrm{C})-\mathrm{C}(24 \mathrm{C})$ & $24.0(6)$ \\
\hline $\mathrm{C}(40 \mathrm{~B})-\mathrm{C}(41 \mathrm{~B})-\mathrm{C}(46 \mathrm{~B})-\mathrm{C}(45 \mathrm{~B})$ & $178.7(5)$ & $C(22 C)-C(23 C)-C(24 C)-C(25 C)$ & $122.4(5)$ \\
\hline $\mathrm{C}(44 \mathrm{~B})-\mathrm{C}(45 \mathrm{~B})-\mathrm{C}(46 \mathrm{~B})-\mathrm{C}(41 \mathrm{~B})$ & $-1.1(9)$ & $\mathrm{C}(22 \mathrm{C})-\mathrm{C}(23 \mathrm{C})-\mathrm{C}(24 \mathrm{C})-\mathrm{C}(20 \mathrm{C})$ & $2.0(5)$ \\
\hline $\mathrm{C}(5 \mathrm{C})-\mathrm{O}(1 \mathrm{C})-\mathrm{C}(4 \mathrm{C})-\mathrm{C}(1 \mathrm{C})$ & $-178.4(4)$ & $\mathrm{N}(4 \mathrm{C})-\mathrm{C}(20 \mathrm{C})-\mathrm{C}(24 \mathrm{C})-\mathrm{C}(25 \mathrm{C})$ & 88.4(4) \\
\hline $\mathrm{C}(5 \mathrm{C})-\mathrm{O}(1 \mathrm{C})-\mathrm{C}(4 \mathrm{C})-\mathrm{C}(2 \mathrm{C})$ & $-60.7(6)$ & $C(21 C)-C(20 C)-C(24 C)-C(25 C)$ & $-150.0(4)$ \\
\hline $\mathrm{C}(5 \mathrm{C})-\mathrm{O}(1 \mathrm{C})-\mathrm{C}(4 \mathrm{C})-\mathrm{C}(3 \mathrm{C})$ & $63.9(6)$ & $\mathrm{N}(4 \mathrm{C})-\mathrm{C}(20 \mathrm{C})-\mathrm{C}(24 \mathrm{C})-\mathrm{C}(23 \mathrm{C})$ & $-148.6(4)$ \\
\hline $\mathrm{C}(6 \mathrm{C})-\mathrm{N}(1 \mathrm{C})-\mathrm{C}(5 \mathrm{C})-\mathrm{O}(2 \mathrm{C})$ & $3.9(7)$ & $C(21 C)-C(20 C)-C(24 C)-C(23 C)$ & $-27.0(5)$ \\
\hline $\mathrm{C}(6 \mathrm{C})-\mathrm{N}(1 \mathrm{C})-\mathrm{C}(5 \mathrm{C})-\mathrm{O}(1 \mathrm{C})$ & $-175.0(4)$ & $\mathrm{C}(26 \mathrm{C})-\mathrm{N}(5 \mathrm{C})-\mathrm{C}(25 \mathrm{C})-\mathrm{O}(6 \mathrm{C})$ & $3.8(7)$ \\
\hline $\mathrm{C}(4 \mathrm{C})-\mathrm{O}(1 \mathrm{C})-\mathrm{C}(5 \mathrm{C})-\mathrm{O}(2 \mathrm{C})$ & $-4.5(7)$ & $\mathrm{C}(26 \mathrm{C})-\mathrm{N}(5 \mathrm{C})-\mathrm{C}(25 \mathrm{C})-\mathrm{C}(24 \mathrm{C})$ & $-179.7(4)$ \\
\hline $\mathrm{C}(4 \mathrm{C})-\mathrm{O}(1 \mathrm{C})-\mathrm{C}(5 \mathrm{C})-\mathrm{N}(1 \mathrm{C})$ & $174.4(4)$ & $\mathrm{C}(23 \mathrm{C})-\mathrm{C}(24 \mathrm{C})-\mathrm{C}(25 \mathrm{C})-\mathrm{O}(6 \mathrm{C})$ & $-20.2(6)$ \\
\hline $\mathrm{C}(5 \mathrm{C})-\mathrm{N}(1 \mathrm{C})-\mathrm{C}(6 \mathrm{C})-\mathrm{C}(7 \mathrm{C})$ & $148.7(4)$ & $\mathrm{C}(20 \mathrm{C})-\mathrm{C}(24 \mathrm{C})-\mathrm{C}(25 \mathrm{C})-\mathrm{O}(6 \mathrm{C})$ & $96.5(5)$ \\
\hline $\mathrm{C}(5 \mathrm{C})-\mathrm{N}(1 \mathrm{C})-\mathrm{C}(6 \mathrm{C})-\mathrm{C}(10 \mathrm{C})$ & $-93.6(5)$ & $\mathrm{C}(23 \mathrm{C})-\mathrm{C}(24 \mathrm{C})-\mathrm{C}(25 \mathrm{C})-\mathrm{N}(5 \mathrm{C})$ & $163.3(4)$ \\
\hline $\mathrm{N}(1 \mathrm{C})-\mathrm{C}(6 \mathrm{C})-\mathrm{C}(7 \mathrm{C})-\mathrm{C}(8 \mathrm{C})$ & $157.5(4)$ & $\mathrm{C}(20 \mathrm{C})-\mathrm{C}(24 \mathrm{C})-\mathrm{C}(25 \mathrm{C})-\mathrm{N}(5 \mathrm{C})$ & $-80.0(5)$ \\
\hline$C(10 C)-C(6 C)-C(7 C)-C(8 C)$ & $32.1(5)$ & $\mathrm{C}(25 \mathrm{C})-\mathrm{N}(5 \mathrm{C})-\mathrm{C}(26 \mathrm{C})-\mathrm{C}(28 \mathrm{C})$ & $70.9(5)$ \\
\hline $\mathrm{C}(6 \mathrm{C})-\mathrm{C}(7 \mathrm{C})-\mathrm{C}(8 \mathrm{C})-\mathrm{C}(9 \mathrm{C})$ & $-13.1(5)$ & $\mathrm{C}(25 \mathrm{C})-\mathrm{N}(5 \mathrm{C})-\mathrm{C}(26 \mathrm{C})-\mathrm{C}(27 \mathrm{C})$ & $-168.8(4)$ \\
\hline $\mathrm{C}(7 \mathrm{C})-\mathrm{C}(8 \mathrm{C})-\mathrm{C}(9 \mathrm{C})-\mathrm{C}(10 \mathrm{C})$ & $-11.7(5)$ & $\mathrm{C}(25 \mathrm{C})-\mathrm{N}(5 \mathrm{C})-\mathrm{C}(26 \mathrm{C})-\mathrm{C}(29 \mathrm{C})$ & $-51.7(5)$ \\
\hline $\mathrm{C}(8 \mathrm{C})-\mathrm{C}(9 \mathrm{C})-\mathrm{C}(10 \mathrm{C})-\mathrm{C}(11 \mathrm{C})$ & $153.1(4)$ & $\mathrm{C}(30 \mathrm{C})-\mathrm{N}(6 \mathrm{C})-\mathrm{C}(29 \mathrm{C})-\mathrm{O}(7 \mathrm{C})$ & $-0.2(7)$ \\
\hline C(8C)-C(9C)-C(10C)-C(6C) & $31.2(5)$ & $C(30 C)-N(6 C)-C(29 C)-C(26 C)$ & $-177.7(4)$ \\
\hline$N(1 C)-C(6 C)-C(10 C)-C(11 C)$ & $77.4(5)$ & $\mathrm{N}(5 \mathrm{C})-\mathrm{C}(26 \mathrm{C})-\mathrm{C}(29 \mathrm{C})-\mathrm{O}(7 \mathrm{C})$ & $144.6(4)$ \\
\hline$C(7 C)-C(6 C)-C(10 C)-C(11 C)$ & $-160.5(4)$ & $\mathrm{C}(28 \mathrm{C})-\mathrm{C}(26 \mathrm{C})-\mathrm{C}(29 \mathrm{C})-\mathrm{O}(7 \mathrm{C})$ & $20.9(6)$ \\
\hline $\mathrm{N}(1 \mathrm{C})-\mathrm{C}(6 \mathrm{C})-\mathrm{C}(10 \mathrm{C})-\mathrm{C}(9 \mathrm{C})$ & $-161.2(4)$ & $\mathrm{C}(27 \mathrm{C})-\mathrm{C}(26 \mathrm{C})-\mathrm{C}(29 \mathrm{C})-\mathrm{O}(7 \mathrm{C})$ & $-97.7(5)$ \\
\hline$C(7 C)-C(6 C)-C(10 C)-C(9 C)$ & $-39.1(5)$ & $\mathrm{N}(5 \mathrm{C})-\mathrm{C}(26 \mathrm{C})-\mathrm{C}(29 \mathrm{C})-\mathrm{N}(6 \mathrm{C})$ & $-37.9(5)$ \\
\hline $\mathrm{C}(12 \mathrm{C})-\mathrm{N}(2 \mathrm{C})-\mathrm{C}(11 \mathrm{C})-\mathrm{O}(3 \mathrm{C})$ & $11.0(7)$ & $\mathrm{C}(28 \mathrm{C})-\mathrm{C}(26 \mathrm{C})-\mathrm{C}(29 \mathrm{C})-\mathrm{N}(6 \mathrm{C})$ & $-161.6(4)$ \\
\hline $\mathrm{C}(12 \mathrm{C})-\mathrm{N}(2 \mathrm{C})-\mathrm{C}(11 \mathrm{C})-\mathrm{C}(10 \mathrm{C})$ & $-171.5(4)$ & $\mathrm{C}(27 \mathrm{C})-\mathrm{C}(26 \mathrm{C})-\mathrm{C}(29 \mathrm{C})-\mathrm{N}(6 \mathrm{C})$ & $79.8(5)$ \\
\hline $\mathrm{C}(9 \mathrm{C})-\mathrm{C}(10 \mathrm{C})-\mathrm{C}(11 \mathrm{C})-\mathrm{O}(3 \mathrm{C})$ & $-30.2(6)$ & $\mathrm{C}(29 \mathrm{C})-\mathrm{N}(6 \mathrm{C})-\mathrm{C}(30 \mathrm{C})-\mathrm{C}(32 \mathrm{C})$ & $67.2(6)$ \\
\hline $\mathrm{C}(6 \mathrm{C})-\mathrm{C}(10 \mathrm{C})-\mathrm{C}(11 \mathrm{C})-\mathrm{O}(3 \mathrm{C})$ & $85.9(5)$ & $\mathrm{C}(29 \mathrm{C})-\mathrm{N}(6 \mathrm{C})-\mathrm{C}(30 \mathrm{C})-\mathrm{C}(31 \mathrm{C})$ & $-172.5(4)$ \\
\hline $\mathrm{C}(9 \mathrm{C})-\mathrm{C}(10 \mathrm{C})-\mathrm{C}(11 \mathrm{C})-\mathrm{N}(2 \mathrm{C})$ & $152.3(4)$ & $\mathrm{C}(29 \mathrm{C})-\mathrm{N}(6 \mathrm{C})-\mathrm{C}(30 \mathrm{C})-\mathrm{C}(33 \mathrm{C})$ & $-56.7(5)$ \\
\hline $\mathrm{C}(6 \mathrm{C})-\mathrm{C}(10 \mathrm{C})-\mathrm{C}(11 \mathrm{C})-\mathrm{N}(2 \mathrm{C})$ & $-91.7(5)$ & $\mathrm{C}(34 \mathrm{C})-\mathrm{N}(7 \mathrm{C})-\mathrm{C}(33 \mathrm{C})-\mathrm{O}(8 \mathrm{C})$ & $2.8(7)$ \\
\hline $\mathrm{C}(11 \mathrm{C})-\mathrm{N}(2 \mathrm{C})-\mathrm{C}(12 \mathrm{C})-\mathrm{C}(13 \mathrm{C})$ & $-169.4(4)$ & $\mathrm{C}(34 \mathrm{C})-\mathrm{N}(7 \mathrm{C})-\mathrm{C}(33 \mathrm{C})-\mathrm{C}(30 \mathrm{C})$ & $-171.2(4)$ \\
\hline $\mathrm{C}(11 \mathrm{C})-\mathrm{N}(2 \mathrm{C})-\mathrm{C}(12 \mathrm{C})-\mathrm{C}(14 \mathrm{C})$ & $71.4(5)$ & $\mathrm{N}(6 \mathrm{C})-\mathrm{C}(30 \mathrm{C})-\mathrm{C}(33 \mathrm{C})-\mathrm{O}(8 \mathrm{C})$ & $159.2(4)$ \\
\hline $\mathrm{C}(11 \mathrm{C})-\mathrm{N}(2 \mathrm{C})-\mathrm{C}(12 \mathrm{C})-\mathrm{C}(15 \mathrm{C})$ & $-50.0(6)$ & $\mathrm{C}(32 \mathrm{C})-\mathrm{C}(30 \mathrm{C})-\mathrm{C}(33 \mathrm{C})-\mathrm{O}(8 \mathrm{C})$ & $35.0(6)$ \\
\hline $\mathrm{C}(16 \mathrm{C})-\mathrm{N}(3 \mathrm{C})-\mathrm{C}(15 \mathrm{C})-\mathrm{O}(4 \mathrm{C})$ & $-0.3(7)$ & $\mathrm{C}(31 \mathrm{C})-\mathrm{C}(30 \mathrm{C})-\mathrm{C}(33 \mathrm{C})-\mathrm{O}(8 \mathrm{C})$ & $-84.0(5)$ \\
\hline$C(16 C)-N(3 C)-C(15 C)-C(12 C)$ & $-177.6(4)$ & $\mathrm{N}(6 \mathrm{C})-\mathrm{C}(30 \mathrm{C})-\mathrm{C}(33 \mathrm{C})-\mathrm{N}(7 \mathrm{C})$ & $-26.6(6)$ \\
\hline $\mathrm{N}(2 \mathrm{C})-\mathrm{C}(12 \mathrm{C})-\mathrm{C}(15 \mathrm{C})-\mathrm{O}(4 \mathrm{C})$ & $145.4(4)$ & $\mathrm{C}(32 \mathrm{C})-\mathrm{C}(30 \mathrm{C})-\mathrm{C}(33 \mathrm{C})-\mathrm{N}(7 \mathrm{C})$ & $-150.8(4)$ \\
\hline $\mathrm{C}(13 \mathrm{C})-\mathrm{C}(12 \mathrm{C})-\mathrm{C}(15 \mathrm{C})-\mathrm{O}(4 \mathrm{C})$ & $-96.3(5)$ & $\mathrm{C}(31 \mathrm{C})-\mathrm{C}(30 \mathrm{C})-\mathrm{C}(33 \mathrm{C})-\mathrm{N}(7 \mathrm{C})$ & $90.2(5)$ \\
\hline $\mathrm{C}(14 \mathrm{C})-\mathrm{C}(12 \mathrm{C})-\mathrm{C}(15 \mathrm{C})-\mathrm{O}(4 \mathrm{C})$ & $23.8(6)$ & $\mathrm{C}(33 \mathrm{C})-\mathrm{N}(7 \mathrm{C})-\mathrm{C}(34 \mathrm{C})-\mathrm{C}(38 \mathrm{C})$ & $-157.1(4)$ \\
\hline
\end{tabular}




\begin{tabular}{|c|c|}
\hline $\mathrm{C}(33 \mathrm{C})-\mathrm{N}(7 \mathrm{C})-\mathrm{C}(34 \mathrm{C})-\mathrm{C}(35 \mathrm{C})$ & $87.7(5)$ \\
\hline $\mathrm{N}(7 \mathrm{C})-\mathrm{C}(34 \mathrm{C})-\mathrm{C}(35 \mathrm{C})-\mathrm{C}(36 \mathrm{C})$ & $80.4(5)$ \\
\hline $\mathrm{C}(38 \mathrm{C})-\mathrm{C}(34 \mathrm{C})-\mathrm{C}(35 \mathrm{C})-\mathrm{C}(36 \mathrm{C})$ & $-38.5(5)$ \\
\hline $\mathrm{C}(34 \mathrm{C})-\mathrm{C}(35 \mathrm{C})-\mathrm{C}(36 \mathrm{C})-\mathrm{C}(37 \mathrm{C})$ & $44.1(6)$ \\
\hline $\mathrm{C}(35 \mathrm{C})-\mathrm{C}(36 \mathrm{C})-\mathrm{C}(37 \mathrm{C})-\mathrm{C}(38 \mathrm{C})$ & $-32.0(6)$ \\
\hline $\mathrm{N}(7 \mathrm{C})-\mathrm{C}(34 \mathrm{C})-\mathrm{C}(38 \mathrm{C})-\mathrm{C}(39 \mathrm{C})$ & $139.1(4)$ \\
\hline $\mathrm{C}(35 \mathrm{C})-\mathrm{C}(34 \mathrm{C})-\mathrm{C}(38 \mathrm{C})-\mathrm{C}(39 \mathrm{C})$ & $-101.1(5)$ \\
\hline N(7C)-C(34C)-C(38C)-C(37C) & $-101.1(5)$ \\
\hline $\mathrm{C}(35 \mathrm{C})-\mathrm{C}(34 \mathrm{C})-\mathrm{C}(38 \mathrm{C})-\mathrm{C}(37 \mathrm{C})$ & $18.7(5)$ \\
\hline $\mathrm{C}(36 \mathrm{C})-\mathrm{C}(37 \mathrm{C})-\mathrm{C}(38 \mathrm{C})-\mathrm{C}(39 \mathrm{C})$ & $131.4(5)$ \\
\hline $\mathrm{C}(36 \mathrm{C})-\mathrm{C}(37 \mathrm{C})-\mathrm{C}(38 \mathrm{C})-\mathrm{C}(34 \mathrm{C})$ & $8.2(6)$ \\
\hline $\mathrm{C}(40 \mathrm{C})-\mathrm{O}(10 \mathrm{C})-\mathrm{C}(39 \mathrm{C})-\mathrm{O}(9 \mathrm{C})$ & $9.2(7)$ \\
\hline $\mathrm{C}(40 \mathrm{C})-\mathrm{O}(10 \mathrm{C})-\mathrm{C}(39 \mathrm{C})-\mathrm{C}(38 \mathrm{C})$ & $-169.5(4)$ \\
\hline $\mathrm{C}(34 \mathrm{C})-\mathrm{C}(38 \mathrm{C})-\mathrm{C}(39 \mathrm{C})-\mathrm{O}(9 \mathrm{C})$ & $5.4(7)$ \\
\hline $\mathrm{C}(37 \mathrm{C})-\mathrm{C}(38 \mathrm{C})-\mathrm{C}(39 \mathrm{C})-\mathrm{O}(9 \mathrm{C})$ & $-113.0(6)$ \\
\hline $\mathrm{C}(34 \mathrm{C})-\mathrm{C}(38 \mathrm{C})-\mathrm{C}(39 \mathrm{C})-\mathrm{O}(10 \mathrm{C})$ & $-176.0(4)$ \\
\hline $\mathrm{C}(37 \mathrm{C})-\mathrm{C}(38 \mathrm{C})-\mathrm{C}(39 \mathrm{C})-\mathrm{O}(10 \mathrm{C})$ & $65.6(5)$ \\
\hline $\mathrm{C}(39 \mathrm{C})-\mathrm{O}(10 \mathrm{C})-\mathrm{C}(40 \mathrm{C})-\mathrm{C}(41 \mathrm{C})$ & $-112.2(6)$ \\
\hline $\mathrm{O}(10 \mathrm{C})-\mathrm{C}(40 \mathrm{C})-\mathrm{C}(41 \mathrm{C})-\mathrm{C}(46 \mathrm{C})$ & $53.3(8)$ \\
\hline $\mathrm{O}(10 \mathrm{C})-\mathrm{C}(40 \mathrm{C})-\mathrm{C}(41 \mathrm{C})-\mathrm{C}(42 \mathrm{C})$ & $-130.0(5)$ \\
\hline$C(46 C)-C(41 C)-C(42 C)-C(43 C)$ & $1.0(8)$ \\
\hline$C(40 C)-C(41 C)-C(42 C)-C(43 C)$ & $-175.9(5)$ \\
\hline$C(41 C)-C(42 C)-C(43 C)-C(44 C)$ & $-0.6(9)$ \\
\hline$C(42 C)-C(43 C)-C(44 C)-C(45 C)$ & $-0.2(9)$ \\
\hline $\mathrm{C}(43 \mathrm{C})-\mathrm{C}(44 \mathrm{C})-\mathrm{C}(45 \mathrm{C})-\mathrm{C}(46 \mathrm{C})$ & $0.5(9)$ \\
\hline $\mathrm{C}(44 \mathrm{C})-\mathrm{C}(45 \mathrm{C})-\mathrm{C}(46 \mathrm{C})-\mathrm{C}(41 \mathrm{C})$ & $-0.1(9)$ \\
\hline $\mathrm{C}(42 \mathrm{C})-\mathrm{C}(41 \mathrm{C})-\mathrm{C}(46 \mathrm{C})-\mathrm{C}(45 \mathrm{C})$ & $-0.7(8)$ \\
\hline$C(40 C)-C(41 C)-C(46 C)-C(45 C)$ & $176.0(6)$ \\
\hline $\mathrm{C}(5 \mathrm{D})-\mathrm{O}(1 \mathrm{D})-\mathrm{C}(4 \mathrm{D})-\mathrm{C}(2 \mathrm{D})$ & $-63.0(6)$ \\
\hline $\mathrm{C}(5 \mathrm{D})-\mathrm{O}(1 \mathrm{D})-\mathrm{C}(4 \mathrm{D})-\mathrm{C}(3 \mathrm{D})$ & $64.1(6)$ \\
\hline $\mathrm{C}(5 \mathrm{D})-\mathrm{O}(1 \mathrm{D})-\mathrm{C}(4 \mathrm{D})-\mathrm{C}(1 \mathrm{D})$ & $-179.1(4)$ \\
\hline $\mathrm{C}(6 \mathrm{D})-\mathrm{N}(1 \mathrm{D})-\mathrm{C}(5 \mathrm{D})-\mathrm{O}(2 \mathrm{D})$ & $-2.3(7)$ \\
\hline $\mathrm{C}(6 \mathrm{D})-\mathrm{N}(1 \mathrm{D})-\mathrm{C}(5 \mathrm{D})-\mathrm{O}(1 \mathrm{D})$ & $178.2(4)$ \\
\hline $\mathrm{C}(4 \mathrm{D})-\mathrm{O}(1 \mathrm{D})-\mathrm{C}(5 \mathrm{D})-\mathrm{O}(2 \mathrm{D})$ & $-0.9(7)$ \\
\hline $\mathrm{C}(4 \mathrm{D})-\mathrm{O}(1 \mathrm{D})-\mathrm{C}(5 \mathrm{D})-\mathrm{N}(1 \mathrm{D})$ & $178.6(4)$ \\
\hline C(5D)-N(1D)-C(6D)-C(7D) & $155.7(5)$ \\
\hline $\mathrm{C}(5 \mathrm{D})-\mathrm{N}(1 \mathrm{D})-\mathrm{C}(6 \mathrm{D})-\mathrm{C}(10 \mathrm{D})$ & $-86.1(6)$ \\
\hline $\mathrm{N}(1 \mathrm{D})-\mathrm{C}(6 \mathrm{D})-\mathrm{C}(7 \mathrm{D})-\mathrm{C}(8 \mathrm{D})$ & $147.1(5)$ \\
\hline $\mathrm{C}(10 \mathrm{D})-\mathrm{C}(6 \mathrm{D})-\mathrm{C}(7 \mathrm{D})-\mathrm{C}(8 \mathrm{D})$ & $23.2(6)$ \\
\hline C(6D)-C(7D)-C(8D)-C(9D) & $-2.7(7)$ \\
\hline C(7D)-C(8D)-C(9D)-C(10D) & $-19.1(6)$ \\
\hline $\mathrm{C}(8 \mathrm{D})-\mathrm{C}(9 \mathrm{D})-\mathrm{C}(10 \mathrm{D})-\mathrm{C}(11 \mathrm{D})$ & $155.4(4)$ \\
\hline C(8D)-C(9D)-C(10D)-C(6D) & $33.1(5)$ \\
\hline $\mathrm{N}(1 \mathrm{D})-\mathrm{C}(6 \mathrm{D})-\mathrm{C}(10 \mathrm{D})-\mathrm{C}(11 \mathrm{D})$ & $81.8(5)$ \\
\hline$C(7 D)-C(6 D)-C(10 D)-C(11 D)$ & $-156.3(4)$ \\
\hline $\mathrm{N}(1 \mathrm{D})-\mathrm{C}(6 \mathrm{D})-\mathrm{C}(10 \mathrm{D})-\mathrm{C}(9 \mathrm{D})$ & $-156.6(4)$ \\
\hline C(7D)-C(6D)-C(10D)-C(9D) & $-34.7(5)$ \\
\hline $\mathrm{C}(12 \mathrm{D})-\mathrm{N}(2 \mathrm{D})-\mathrm{C}(11 \mathrm{D})-\mathrm{O}(3 \mathrm{D})$ & $11.1(7)$ \\
\hline $\mathrm{C}(12 \mathrm{D})-\mathrm{N}(2 \mathrm{D})-\mathrm{C}(11 \mathrm{D})-\mathrm{C}(10 \mathrm{D})$ & $-169.5(4)$ \\
\hline $\mathrm{C}(9 \mathrm{D})-\mathrm{C}(10 \mathrm{D})-\mathrm{C}(11 \mathrm{D})-\mathrm{O}(3 \mathrm{D})$ & $-35.5(6)$ \\
\hline$C(6 D)-C(10 D)-C(11 D)-O(3 D)$ & $81.3(6)$ \\
\hline $\mathrm{C}(9 \mathrm{D})-\mathrm{C}(10 \mathrm{D})-\mathrm{C}(11 \mathrm{D})-\mathrm{N}(2 \mathrm{D})$ & $145.1(4)$ \\
\hline $\mathrm{C}(6 \mathrm{D})-\mathrm{C}(10 \mathrm{D})-\mathrm{C}(11 \mathrm{D})-\mathrm{N}(2 \mathrm{D})$ & $-98.1(5)$ \\
\hline $\mathrm{C}(11 \mathrm{D})-\mathrm{N}(2 \mathrm{D})-\mathrm{C}(12 \mathrm{D})-\mathrm{C}(13 \mathrm{D})$ & $-170.3(4)$ \\
\hline $\mathrm{C}(11 \mathrm{D})-\mathrm{N}(2 \mathrm{D})-\mathrm{C}(12 \mathrm{D})-\mathrm{C}(15 \mathrm{D})$ & $-51.7(6)$ \\
\hline
\end{tabular}

\begin{tabular}{|c|c|}
\hline $\mathrm{C}(11 \mathrm{D})-\mathrm{N}(2 \mathrm{D})-\mathrm{C}(12 \mathrm{D})-\mathrm{C}(14 \mathrm{D})$ & $69.4(5)$ \\
\hline $\mathrm{C}(16 \mathrm{D})-\mathrm{N}(3 \mathrm{D})-\mathrm{C}(15 \mathrm{D})-\mathrm{O}(4 \mathrm{D})$ & $-0.5(7)$ \\
\hline $\mathrm{C}(16 \mathrm{D})-\mathrm{N}(3 \mathrm{D})-\mathrm{C}(15 \mathrm{D})-\mathrm{C}(12 \mathrm{D})$ & $-176.4(4)$ \\
\hline $\mathrm{N}(2 \mathrm{D})-\mathrm{C}(12 \mathrm{D})-\mathrm{C}(15 \mathrm{D})-\mathrm{O}(4 \mathrm{D})$ & $144.7(4)$ \\
\hline $\mathrm{C}(13 \mathrm{D})-\mathrm{C}(12 \mathrm{D})-\mathrm{C}(15 \mathrm{D})-\mathrm{O}(4 \mathrm{D})$ & $-97.2(5)$ \\
\hline $\mathrm{C}(14 \mathrm{D})-\mathrm{C}(12 \mathrm{D})-\mathrm{C}(15 \mathrm{D})-\mathrm{O}(4 \mathrm{D})$ & $23.9(6)$ \\
\hline $\mathrm{N}(2 \mathrm{D})-\mathrm{C}(12 \mathrm{D})-\mathrm{C}(15 \mathrm{D})-\mathrm{N}(3 \mathrm{D})$ & $-39.4(6)$ \\
\hline $\mathrm{C}(13 \mathrm{D})-\mathrm{C}(12 \mathrm{D})-\mathrm{C}(15 \mathrm{D})-\mathrm{N}(3 \mathrm{D})$ & $78.7(5)$ \\
\hline $\mathrm{C}(14 \mathrm{D})-\mathrm{C}(12 \mathrm{D})-\mathrm{C}(15 \mathrm{D})-\mathrm{N}(3 \mathrm{D})$ & $-160.2(4)$ \\
\hline $\mathrm{C}(15 \mathrm{D})-\mathrm{N}(3 \mathrm{D})-\mathrm{C}(16 \mathrm{D})-\mathrm{C}(18 \mathrm{D})$ & $66.0(6)$ \\
\hline $\mathrm{C}(15 \mathrm{D})-\mathrm{N}(3 \mathrm{D})-\mathrm{C}(16 \mathrm{D})-\mathrm{C}(17 \mathrm{D})$ & $-173.1(4)$ \\
\hline $\mathrm{C}(15 \mathrm{D})-\mathrm{N}(3 \mathrm{D})-\mathrm{C}(16 \mathrm{D})-\mathrm{C}(19 \mathrm{D})$ & $-57.2(5)$ \\
\hline $\mathrm{C}(20 \mathrm{D})-\mathrm{N}(4 \mathrm{D})-\mathrm{C}(19 \mathrm{D})-\mathrm{O}(5 \mathrm{D})$ & $-11.1(7)$ \\
\hline$C(20 D)-N(4 D)-C(19 D)-C(16 D)$ & $174.9(4)$ \\
\hline $\mathrm{N}(3 \mathrm{D})-\mathrm{C}(16 \mathrm{D})-\mathrm{C}(19 \mathrm{D})-\mathrm{O}(5 \mathrm{D})$ & $154.9(4)$ \\
\hline $\mathrm{C}(18 \mathrm{D})-\mathrm{C}(16 \mathrm{D})-\mathrm{C}(19 \mathrm{D})-\mathrm{O}(5 \mathrm{D})$ & $31.2(6)$ \\
\hline $\mathrm{C}(17 \mathrm{D})-\mathrm{C}(16 \mathrm{D})-\mathrm{C}(19 \mathrm{D})-\mathrm{O}(5 \mathrm{D})$ & $-87.9(5)$ \\
\hline $\mathrm{N}(3 \mathrm{D})-\mathrm{C}(16 \mathrm{D})-\mathrm{C}(19 \mathrm{D})-\mathrm{N}(4 \mathrm{D})$ & $-30.8(5)$ \\
\hline $\mathrm{C}(18 \mathrm{D})-\mathrm{C}(16 \mathrm{D})-\mathrm{C}(19 \mathrm{D})-\mathrm{N}(4 \mathrm{D})$ & $-154.4(4)$ \\
\hline$C(17 D)-C(16 D)-C(19 D)-N(4 D)$ & $86.4(5)$ \\
\hline $\mathrm{C}(19 \mathrm{D})-\mathrm{N}(4 \mathrm{D})-\mathrm{C}(20 \mathrm{D})-\mathrm{C}(21 \mathrm{D})$ & $147.2(4)$ \\
\hline $\mathrm{C}(19 \mathrm{D})-\mathrm{N}(4 \mathrm{D})-\mathrm{C}(20 \mathrm{D})-\mathrm{C}(24 \mathrm{D})$ & $-95.2(5)$ \\
\hline $\mathrm{N}(4 \mathrm{D})-\mathrm{C}(20 \mathrm{D})-\mathrm{C}(21 \mathrm{D})-\mathrm{C}(22 \mathrm{D})$ & $121.1(4)$ \\
\hline$C(24 D)-C(20 D)-C(21 D)-C(22 D)$ & $-0.8(5)$ \\
\hline$C(20 D)-C(21 D)-C(22 D)-C(23 D)$ & $25.4(5)$ \\
\hline $\mathrm{C}(21 \mathrm{D})-\mathrm{C}(22 \mathrm{D})-\mathrm{C}(23 \mathrm{D})-\mathrm{C}(24 \mathrm{D})$ & $-40.4(5)$ \\
\hline $\mathrm{C}(22 \mathrm{D})-\mathrm{C}(23 \mathrm{D})-\mathrm{C}(24 \mathrm{D})-\mathrm{C}(25 \mathrm{D})$ & $161.7(4)$ \\
\hline$C(22 D)-C(23 D)-C(24 D)-C(20 D)$ & $39.5(5)$ \\
\hline $\mathrm{N}(4 \mathrm{D})-\mathrm{C}(20 \mathrm{D})-\mathrm{C}(24 \mathrm{D})-\mathrm{C}(25 \mathrm{D})$ & $89.2(4)$ \\
\hline$C(21 D)-C(20 D)-C(24 D)-C(25 D)$ & $-148.6(4)$ \\
\hline $\mathrm{N}(4 \mathrm{D})-\mathrm{C}(20 \mathrm{D})-\mathrm{C}(24 \mathrm{D})-\mathrm{C}(23 \mathrm{D})$ & $-146.4(4)$ \\
\hline$C(21 D)-C(20 D)-C(24 D)-C(23 D)$ & $-24.3(5)$ \\
\hline $\mathrm{C}(26 \mathrm{D})-\mathrm{N}(5 \mathrm{D})-\mathrm{C}(25 \mathrm{D})-\mathrm{O}(6 \mathrm{D})$ & $4.7(7)$ \\
\hline $\mathrm{C}(26 \mathrm{D})-\mathrm{N}(5 \mathrm{D})-\mathrm{C}(25 \mathrm{D})-\mathrm{C}(24 \mathrm{D})$ & $-179.1(4)$ \\
\hline $\mathrm{C}(23 \mathrm{D})-\mathrm{C}(24 \mathrm{D})-\mathrm{C}(25 \mathrm{D})-\mathrm{O}(6 \mathrm{D})$ & $-23.0(6)$ \\
\hline $\mathrm{C}(20 \mathrm{D})-\mathrm{C}(24 \mathrm{D})-\mathrm{C}(25 \mathrm{D})-\mathrm{O}(6 \mathrm{D})$ & $95.8(5)$ \\
\hline $\mathrm{C}(23 \mathrm{D})-\mathrm{C}(24 \mathrm{D})-\mathrm{C}(25 \mathrm{D})-\mathrm{N}(5 \mathrm{D})$ & $160.8(4)$ \\
\hline $\mathrm{C}(20 \mathrm{D})-\mathrm{C}(24 \mathrm{D})-\mathrm{C}(25 \mathrm{D})-\mathrm{N}(5 \mathrm{D})$ & $-80.4(5)$ \\
\hline $\mathrm{C}(25 \mathrm{D})-\mathrm{N}(5 \mathrm{D})-\mathrm{C}(26 \mathrm{D})-\mathrm{C}(28 \mathrm{D})$ & $68.5(5)$ \\
\hline $\mathrm{C}(25 \mathrm{D})-\mathrm{N}(5 \mathrm{D})-\mathrm{C}(26 \mathrm{D})-\mathrm{C}(27 \mathrm{D})$ & $-171.6(4)$ \\
\hline $\mathrm{C}(25 \mathrm{D})-\mathrm{N}(5 \mathrm{D})-\mathrm{C}(26 \mathrm{D})-\mathrm{C}(29 \mathrm{D})$ & $-53.6(5)$ \\
\hline $\mathrm{C}(30 \mathrm{D})-\mathrm{N}(6 \mathrm{D})-\mathrm{C}(29 \mathrm{D})-\mathrm{O}(7 \mathrm{D})$ & $-0.3(7)$ \\
\hline$C(30 D)-N(6 D)-C(29 D)-C(26 D)$ & $-177.4(4)$ \\
\hline $\mathrm{N}(5 \mathrm{D})-\mathrm{C}(26 \mathrm{D})-\mathrm{C}(29 \mathrm{D})-\mathrm{O}(7 \mathrm{D})$ & $147.3(4)$ \\
\hline $\mathrm{C}(28 \mathrm{D})-\mathrm{C}(26 \mathrm{D})-\mathrm{C}(29 \mathrm{D})-\mathrm{O}(7 \mathrm{D})$ & $24.7(6)$ \\
\hline$C(27 D)-C(26 D)-C(29 D)-O(7 D)$ & $-94.5(5)$ \\
\hline$N(5 D)-C(26 D)-C(29 D)-N(6 D)$ & $-35.5(5)$ \\
\hline $\mathrm{C}(28 \mathrm{D})-\mathrm{C}(26 \mathrm{D})-\mathrm{C}(29 \mathrm{D})-\mathrm{N}(6 \mathrm{D})$ & $-158.0(4)$ \\
\hline $\mathrm{C}(27 \mathrm{D})-\mathrm{C}(26 \mathrm{D})-\mathrm{C}(29 \mathrm{D})-\mathrm{N}(6 \mathrm{D})$ & $82.7(5)$ \\
\hline $\mathrm{C}(29 \mathrm{D})-\mathrm{N}(6 \mathrm{D})-\mathrm{C}(30 \mathrm{D})-\mathrm{C}(32 \mathrm{D})$ & $62.8(5)$ \\
\hline$C(29 D)-N(6 D)-C(30 D)-C(31 D)$ & $-177.2(4)$ \\
\hline $\mathrm{C}(29 \mathrm{D})-\mathrm{N}(6 \mathrm{D})-\mathrm{C}(30 \mathrm{D})-\mathrm{C}(33 \mathrm{D})$ & $-62.2(5)$ \\
\hline $\mathrm{C}(34 \mathrm{D})-\mathrm{N}(7 \mathrm{D})-\mathrm{C}(33 \mathrm{D})-\mathrm{O}(8 \mathrm{D})$ & $1.4(7)$ \\
\hline $\mathrm{C}(34 \mathrm{D})-\mathrm{N}(7 \mathrm{D})-\mathrm{C}(33 \mathrm{D})-\mathrm{C}(30 \mathrm{D})$ & $-171.0(4)$ \\
\hline $\mathrm{N}(6 \mathrm{D})-\mathrm{C}(30 \mathrm{D})-\mathrm{C}(33 \mathrm{D})-\mathrm{O}(8 \mathrm{D})$ & $163.0(4)$ \\
\hline
\end{tabular}




$\begin{array}{lc} & \\ \text { C(32D)-C(30D)-C(33D)-O(8D) } & 37.5(5) \\ \text { C(31D)-C(30D)-C(33D)-O(8D) } & -81.4(5) \\ \text { N(6D)-C(30D)-C(33D)-N(7D) } & -24.2(5) \\ \text { C(32D)-C(30D)-C(33D)-N(7D) } & -149.8(4) \\ \text { C(31D)-C(30D)-C(33D)-N(7D) } & 91.3(5) \\ \text { C(33D)-N(7D)-C(34D)-C(35D) } & 95.3(5) \\ \text { C(33D)-N(7D)-C(34D)-C(38D) } & -149.1(4) \\ \text { N(7D)-C(34D)-C(35D)-C(36D) } & 81.3(4) \\ \text { C(38D)-C(34D)-C(35D)-C(36D) } & -37.3(5) \\ \text { C(34D)-C(35D)-C(36D)-C(37D) } & 43.2(5) \\ \text { C(35D)-C(36D)-C(37D)-C(38D) } & -32.2(5) \\ \text { N(7D)-C(34D)-C(38D)-C(39D) } & 139.8(4) \\ \text { C(35D)-C(34D)-C(38D)-C(39D) } & -101.0(4) \\ \text { N(7D)-C(34D)-C(38D)-C(37D) } & -102.0(4) \\ \text { C(35D)-C(34D)-C(38D)-C(37D) } & 17.2(5) \\ \text { C(36D)-C(37D)-C(38D)-C(39D) } & 129.8(4) \\ \text { C(36D)-C(37D)-C(38D)-C(34D) } & 9.3(5) \\ \text { C(40D)-O(10D)-C(39D)-O(9D) } & 0.6(7) \\ \text { C(40D)-O(10D)-C(39D)-C(38D) } & -177.7(4) \\ \text { C(34D)-C(38D)-C(39D)-O(9D) } & 16.2(7) \\ \text { C(37D)-C(38D)-C(39D)-O(9D) } & -100.0(5) \\ \text { C(34D)-C(38D)-C(39D)-O(10D) } & -165.5(4) \\ \text { C(37D)-C(38D)-C(39D)-O(10D) } & 78.2(5) \\ \text { C(39D)-O(10D)-C(40D)-C(41D) } & -178.4(4) \\ \text { O(10D)-C(40D)-C(41D)-C(42D) } & -82.0(6) \\ \text { O(10D)-C(40D)-C(41D)-C(46D) } & 99.1(5) \\ \text { C(46D)-C(41D)-C(42D)-C(43D) } & 0.0(8) \\ \text { C(40D)-C(41D)-C(42D)-C(43D) } & -178.9(5) \\ \text { C(41D)-C(42D)-C(43D)-C(44D) } & 0.2(9) \\ \text { C(42D)-C(43D)-C(44D)-C(45D) } & 0.5(9) \\ \text { C(43D)-C(44D)-C(45D)-C(46D) } & -1.3(9) \\ \text { C(42D)-C(41D)-C(46D)-C(45D) } & -0.8(7) \\ \text { C(40D)-C(41D)-C(46D)-C(45D) } & 178.1(5) \\ \text { C(44D)-C(45D)-C(46D)-C(41D) } & 1.4(8) \\ \text { C(5E)-O(1E)-C(4E)-C(2E) } & -61.0(6) \\ \text { C(5E)-O(1E)-C(4E)-C(1E) } & 65.0(6) \\ \text { C(5E)-O(1E)-C(4E)-C(3E) } & -177.6(5) \\ \text { C(6E)-N(1E)-C(5E)-O(2E) } & 4.9(7) \\ \text { C(6E)-N(1E)-C(5E)-O(1E) } & -175.0(4) \\ \text { C(4E)-O(1E)-C(5E)-O(2E) } & -7.3(7) \\ \text { C(4E)-O(1E)-C(5E)-N(1E) } & 172.6(4) \\ \text { C(5E)-N(1E)-C(6E)-C(7E) } & 147.4(4) \\ \text { C(5E)-N(1E)-C(6E)-C(10E) } & -94.3(5) \\ \text { N(1E)-C(6E)-C(7E)-C(8E) } & 152.4(4) \\ \text { C(10E)-C(6E)-C(7E)-C(8E) } & 27.1(5) \\ \text { C(6E)-C(7E)-C(8E)-C(9E) } & -4.9(5) \\ \text { C(7E)-C(8E)-C(9E)-C(10E) } & -19.6(5) \\ \text { C(8E)-C(9E)-C(10E)-C(11E) } & 157.7(4) \\ \text { C(8E)-C(9E)-C(10E)-C(6E) } & 35.8(5) \\ \text { N(1E)-C(6E)-C(10E)-C(11E) } & 78.8(5) \\ \text { C(7E)-C(6E)-C(10E)-C(11E) } & -159.4(4) \\ \text { N(1E)-C(6E)-C(10E)-C(9E) } & -160.0(4) \\ \text { C(7E)-C(6E)-C(10E)-C(9E) } & -38.3(4) \\ \text { C(12E)-N(2E)-C(11E)-O(3E) } & 9.2(7) \\ \text { C(12E)-N(2E)-C(11E)-C(10E) } & -171.4(4) \\ & \\ & \end{array}$

\begin{abstract}
$\mathrm{C}(9 \mathrm{E})-\mathrm{C}(10 \mathrm{E})-\mathrm{C}(11 \mathrm{E})-\mathrm{O}(3 \mathrm{E})$
\end{abstract}
$\mathrm{C}(6 \mathrm{E})-\mathrm{C}(10 \mathrm{E})-\mathrm{C}(11 \mathrm{E})-\mathrm{O}(3 \mathrm{E})$

C(9E)-C(10E)-C(11E)-N(2E)

$\mathrm{C}(6 \mathrm{E})-\mathrm{C}(10 \mathrm{E})-\mathrm{C}(11 \mathrm{E})-\mathrm{N}(2 \mathrm{E})$

C(11E)-N(2E)-C(12E)-C(13E)

C(11E)-N(2E)-C(12E)-C(14E)

C(11E)-N(2E)-C(12E)-C(15E)

C(16E)-N(3E)-C(15E)-O(4E)

C(16E)-N(3E)-C(15E)-C(12E)

$\mathrm{N}(2 \mathrm{E})-\mathrm{C}(12 \mathrm{E})-\mathrm{C}(15 \mathrm{E})-\mathrm{O}(4 \mathrm{E})$

$\mathrm{C}(13 \mathrm{E})-\mathrm{C}(12 \mathrm{E})-\mathrm{C}(15 \mathrm{E})-\mathrm{O}(4 \mathrm{E})$

C(14E)-C(12E)-C(15E)-O(4E)

$\mathrm{N}(2 \mathrm{E})-\mathrm{C}(12 \mathrm{E})-\mathrm{C}(15 \mathrm{E})-\mathrm{N}(3 \mathrm{E})$

$\mathrm{C}(13 \mathrm{E})-\mathrm{C}(12 \mathrm{E})-\mathrm{C}(15 \mathrm{E})-\mathrm{N}(3 \mathrm{E})$

C(14E)-C(12E)-C(15E)-N(3E)

C(15E)-N(3E)-C(16E)-C(18E)

C(15E)-N(3E)-C(16E)-C(17E)

C(15E)-N(3E)-C(16E)-C(19E)

C(20E)-N(4E)-C(19E)-O(5E)

C(20E)-N(4E)-C(19E)-C(16E)

$\mathrm{N}(3 \mathrm{E})-\mathrm{C}(16 \mathrm{E})-\mathrm{C}(19 \mathrm{E})-\mathrm{O}(5 \mathrm{E})$

C(18E)-C(16E)-C(19E)-O(5E)

C(17E)-C(16E)-C(19E)-O(5E)

$\mathrm{N}(3 \mathrm{E})-\mathrm{C}(16 \mathrm{E})-\mathrm{C}(19 \mathrm{E})-\mathrm{N}(4 \mathrm{E})$

C(18E)-C(16E)-C(19E)-N(4E)

C(17E)-C(16E)-C(19E)-N(4E)

C(19E)-N(4E)-C(20E)-C(21E)

C(19E)-N(4E)-C(20E)-C(24E)

$\mathrm{N}(4 \mathrm{E})-\mathrm{C}(20 \mathrm{E})-\mathrm{C}(21 \mathrm{E})-\mathrm{C}(22 \mathrm{E})$

$\mathrm{C}(24 \mathrm{E})-\mathrm{C}(20 \mathrm{E})-\mathrm{C}(21 \mathrm{E})-\mathrm{C}(22 \mathrm{E})$

$\mathrm{C}(20 \mathrm{E})-\mathrm{C}(21 \mathrm{E})-\mathrm{C}(22 \mathrm{E})-\mathrm{C}(23 \mathrm{E})$

$\mathrm{C}(21 \mathrm{E})-\mathrm{C}(22 \mathrm{E})-\mathrm{C}(23 \mathrm{E})-\mathrm{C}(24 \mathrm{E})$

$\mathrm{C}(22 \mathrm{E})-\mathrm{C}(23 \mathrm{E})-\mathrm{C}(24 \mathrm{E})-\mathrm{C}(25 \mathrm{E})$

$\mathrm{C}(22 \mathrm{E})-\mathrm{C}(23 \mathrm{E})-\mathrm{C}(24 \mathrm{E})-\mathrm{C}(20 \mathrm{E})$

$\mathrm{N}(4 \mathrm{E})-\mathrm{C}(20 \mathrm{E})-\mathrm{C}(24 \mathrm{E})-\mathrm{C}(25 \mathrm{E})$

$\mathrm{C}(21 \mathrm{E})-\mathrm{C}(20 \mathrm{E})-\mathrm{C}(24 \mathrm{E})-\mathrm{C}(25 \mathrm{E})$

$\mathrm{N}(4 \mathrm{E})-\mathrm{C}(20 \mathrm{E})-\mathrm{C}(24 \mathrm{E})-\mathrm{C}(23 \mathrm{E})$

$\mathrm{C}(21 \mathrm{E})-\mathrm{C}(20 \mathrm{E})-\mathrm{C}(24 \mathrm{E})-\mathrm{C}(23 \mathrm{E})$

$\mathrm{C}(26 \mathrm{E})-\mathrm{N}(5 \mathrm{E})-\mathrm{C}(25 \mathrm{E})-\mathrm{O}(6 \mathrm{E})$

C(26E)-N(5E)-C(25E)-C(24E)

$\mathrm{C}(23 \mathrm{E})-\mathrm{C}(24 \mathrm{E})-\mathrm{C}(25 \mathrm{E})-\mathrm{O}(6 \mathrm{E})$

$\mathrm{C}(20 \mathrm{E})-\mathrm{C}(24 \mathrm{E})-\mathrm{C}(25 \mathrm{E})-\mathrm{O}(6 \mathrm{E})$

$\mathrm{C}(23 \mathrm{E})-\mathrm{C}(24 \mathrm{E})-\mathrm{C}(25 \mathrm{E})-\mathrm{N}(5 \mathrm{E})$

$\mathrm{C}(20 \mathrm{E})-\mathrm{C}(24 \mathrm{E})-\mathrm{C}(25 \mathrm{E})-\mathrm{N}(5 \mathrm{E})$

C(25E)-N(5E)-C(26E)-C(28E)

$\mathrm{C}(25 \mathrm{E})-\mathrm{N}(5 \mathrm{E})-\mathrm{C}(26 \mathrm{E})-\mathrm{C}(27 \mathrm{E})$

$\mathrm{C}(25 \mathrm{E})-\mathrm{N}(5 \mathrm{E})-\mathrm{C}(26 \mathrm{E})-\mathrm{C}(29 \mathrm{E})$

C(30E)-N(6E)-C(29E)-O(7E)

C(30E)-N(6E)-C(29E)-C(26E)

$\mathrm{N}(5 \mathrm{E})-\mathrm{C}(26 \mathrm{E})-\mathrm{C}(29 \mathrm{E})-\mathrm{O}(7 \mathrm{E})$

C(28E)-C(26E)-C(29E)-O(7E)

C(27E)-C(26E)-C(29E)-O(7E)

$\mathrm{N}(5 \mathrm{E})-\mathrm{C}(26 \mathrm{E})-\mathrm{C}(29 \mathrm{E})-\mathrm{N}(6 \mathrm{E})$

$\mathrm{C}(28 \mathrm{E})-\mathrm{C}(26 \mathrm{E})-\mathrm{C}(29 \mathrm{E})-\mathrm{N}(6 \mathrm{E})$

$\mathrm{C}(27 \mathrm{E})-\mathrm{C}(26 \mathrm{E})-\mathrm{C}(29 \mathrm{E})-\mathrm{N}(6 \mathrm{E})$
$-24.9(6)$

$89.9(5)$

155.7(4)

$-89.5(5)$

$-169.8(4)$

69.5(5)

$-51.9(6)$

$0.6(7)$

$-176.7(4)$

143.2(4)

$-99.2(5)$

20.7(6)

$-39.4(5)$

78.2(5)

$-161.9(4)$

67.1(6)

$-171.9(4)$

$-55.2(5)$

$-6.6(7)$

178.5(4)

149.1(4)

26.8(6)

$-93.6(5)$

$-35.8(5)$

$-158.0(4)$

81.5(5)

144.3(5)

$-99.2(5)$

161.5(4)

$38.9(5)$

$-37.1(5)$

21.0(5)

124.3(4)

2.7(5)

$89.9(5)$

$-149.0(4)$

$-146.6(4)$

$-25.5(5)$

5.2(7)

$-177.3(4)$

$-19.7(7)$

98.9(5)

162.8(4)

$-78.6(5)$

69.9(6)

$-169.4(4)$

$-51.8(6)$

2.8(7)

$-175.6(4)$

143.3(5)

21.8(6)

$-99.0(5)$

$-38.3(6)$

$-159.8(4)$

79.4(5) 


$\begin{array}{lc}\text { C(29E)-N(6E)-C(30E)-C(32E) } & 61.2(6) \\ \text { C(29E)-N(6E)-C(30E)-C(33E) } & -62.4(6) \\ \text { C(29E)-N(6E)-C(30E)-C(31E) } & -179.0(4) \\ \text { C(34E)-N(7E)-C(33E)-O(8E) } & 0.8(7) \\ \text { C(34E)-N(7E)-C(33E)-C(30E) } & -174.0(4) \\ \text { N(6E)-C(30E)-C(33E)-O(8E) } & 162.9(4) \\ \text { C(32E)-C(30E)-C(33E)-O(8E) } & 38.9(6) \\ \text { C(31E)-C(30E)-C(33E)-O(8E) } & -80.9(5) \\ \text { N(6E)-C(30E)-C(33E)-N(7E) } & -22.1(6) \\ \text { C(32E)-C(30E)-C(33E)-N(7E) } & -146.0(4) \\ \text { C(31E)-C(30E)-C(33E)-N(7E) } & 94.1(5) \\ \text { C(33E)-N(7E)-C(34E)-C(35E) } & 98.9(5) \\ \text { C(33E)-N(7E)-C(34E)-C(38E) } & -146.5(4) \\ \text { N(7E)-C(34E)-C(35E)-C(36E) } & 79.3(5) \\ \text { C(38E)-C(34E)-C(35E)-C(36E) } & -38.3(5) \\ \text { C(34E)-C(35E)-C(36E)-C(37E) } & 43.5(5) \\ \text { C(35E)-C(36E)-C(37E)-C(38E) } & -31.6(5) \\ \text { N(7E)-C(34E)-C(38E)-C(39E) } & 143.2(4) \\ \text { C(35E)-C(34E)-C(38E)-C(39E) } & -98.4(4) \\ \text { N(7E)-C(34E)-C(38E)-C(37E) } & -99.6(4) \\ \text { C(35E)-C(34E)-C(38E)-C(37E) } & 18.8(5) \\ \text { C(36E)-C(37E)-C(38E)-C(39E) } & 127.7(4) \\ \text { C(36E)-C(37E)-C(38E)-C(34E) } & 8.0(5) \\ \text { C(40E)-O(10E)-C(39E)-O(9E) } & 3.6(7) \\ \text { C(40E)-O(10E)-C(39E)-C(38E) } & -172.7(4) \\ \text { C(34E)-C(38E)-C(39E)-O(9E) } & 32.3(7) \\ \text { C(37E)-C(38E)-C(39E)-O(9E) } & -83.8(6) \\ \text { C(34E)-C(38E)-C(39E)-O(10E) } & -151.6(4) \\ \text { C(37E)-C(38E)-C(39E)-O(10E) } & 92.4(5) \\ \text { C(39E)-O(10E)-C(40E)-C(41E) } & 169.9(4) \\ \text { O(10E)-C(40E)-C(41E)-C(46E) } & 108.7(5) \\ \text { O(10E)-C(40E)-C(41E)-C(42E) } & -71.7(6) \\ \text { C(46E)-C(41E)-C(42E)-C(43E) } & 1.9(7) \\ \text { C(40E)-C(41E)-C(42E)-C(43E) } & -177.6(5) \\ \text { C(41E)-C(42E)-C(43E)-C(44E) } & -0.2(8) \\ \text { C(42E)-C(43E)-C(44E)-C(45E) } & -1.1(8) \\ \text { C(43E)-C(44E)-C(45E)-C(46E) } & 0.7(8) \\ \text { C(42E)-C(41E)-C(46E)-C(45E) } & -2.3(7) \\ \text { C(40E)-C(41E)-C(46E)-C(45E) } & 177.3(5) \\ \text { C(44E)-C(45E)-C(46E)-C(41E) } & 1.0(8) \\ \text { C(5F)-O(1F)-C(4F)-C(3F) } & 61.8(6) \\ \text { C(5F)-O(1F)-C(4F)-C(1F) } & -179.4(4) \\ \text { C(5F)-O(1F)-C(4F)-C(2F) } & -61.9(5) \\ \text { C(6F)-N(1F)-C(5F)-O(2F) } & -1.8(7) \\ \text { C(6F)-N(1F)-C(5F)-O(1F) } & 178.2(4) \\ \text { C(4F)-O(1F)-C(5F)-O(2F) } & -5.2(7) \\ \text { C(4F)-O(1F)-C(5F)-N(1F) } & 174.8(4) \\ \text { C(5F)-N(1F)-C(6F)-C(7F) } & 149.6(4) \\ \text { C(5F)-N(1F)-C(6F)-C(10F) } & -90.5(5) \\ \text { N(1F)-C(6F)-C(7F)-C(8F) } & 167.5(4) \\ \text { C(10F)-C(6F)-C(7F)-C(8F) } & 42.5(5) \\ \text { C(6F)-C(7F)-C(8F)-C(9F) } & -43.6(5) \\ \text { C(7F)-C(8F)-C(9F)-C(10F) } & 28.4(5) \\ \text { N(1F)-C(6F)-C(10F)-C(11F) } & 89.5(5) \\ \text { C(7F)-C(6F)-C(10F)-C(11F) } & -146.1(4) \\ & \\ & \end{array}$

$\begin{array}{lc} & \\ \text { N(1F)-C(6F)-C(10F)-C(9F) } & -149.7(4) \\ \text { C(7F)-C(6F)-C(10F)-C(9F) } & -25.2(5) \\ \text { C(8F)-C(9F)-C(10F)-C(11F) } & 118.8(4) \\ \text { C(8F)-C(9F)-C(10F)-C(6F) } & -1.9(5) \\ \text { C(12F)-N(2F)-C(11F)-O(3F) } & 7.7(7) \\ \text { C(12F)-N(2F)-C(11F)-C(10F) } & -173.9(4) \\ \text { C(6F)-C(10F)-C(11F)-O(3F) } & 83.0(5) \\ \text { C(9F)-C(10F)-C(11F)-O(3F) } & -33.3(6) \\ \text { C(6F)-C(10F)-C(11F)-N(2F) } & -95.5(5) \\ \text { C(9F)-C(10F)-C(11F)-N(2F) } & 148.2(4) \\ \text { C(11F)-N(2F)-C(12F)-C(13F) } & -168.5(4) \\ \text { C(11F)-N(2F)-C(12F)-C(15F) } & -49.3(6) \\ \text { C(11F)-N(2F)-C(12F)-C(14F) } & 71.7(5) \\ \text { C(16F)-N(3F)-C(15F)-O(4F) } & -2.5(7) \\ \text { C(16F)-N(3F)-C(15F)-C(12F) } & -177.2(4) \\ \text { N(2F)-C(12F)-C(15F)-O(4F) } & 142.2(4) \\ \text { C(13F)-C(12F)-C(15F)-O(4F) } & -99.8(5) \\ \text { C(14F)-C(12F)-C(15F)-O(4F) } & 21.1(6) \\ \text { N(2F)-C(12F)-C(15F)-N(3F) } & -43.1(6) \\ \text { C(13F)-C(12F)-C(15F)-N(3F) } & 74.9(5) \\ \text { C(14F)-C(12F)-C(15F)-N(3F) } & -164.2(4) \\ \text { C(15F)-N(3F)-C(16F)-C(18F) } & 69.2(5) \\ \text { C(15F)-N(3F)-C(16F)-C(17F) } & -170.6(4) \\ \text { C(15F)-N(3F)-C(16F)-C(19F) } & -53.0(6) \\ \text { C(20F)-N(4F)-C(19F)-O(5F) } & -6.8(7) \\ \text { C(20F)-N(4F)-C(19F)-C(16F) } & 179.2(4) \\ \text { N(3F)-C(16F)-C(19F)-O(5F) } & 148.8(4) \\ \text { C(18F)-C(16F)-C(19F)-O(5F) } & 25.4(6) \\ \text { C(17F)-C(16F)-C(19F)-O(5F) } & -93.1(5) \\ \text { N(3F)-C(16F)-C(19F)-N(4F) } & -36.9(5) \\ \text { C(18F)-C(16F)-C(19F)-N(4F) } & -160.3(4) \\ \text { C(17F)-C(16F)-C(19F)-N(4F) } & 81.2(5) \\ \text { C(19F)-N(4F)-C(20F)-C(21F) } & 144.6(4) \\ \text { C(19F)-N(4F)-C(20F)-C(24F) } & -98.7(5) \\ \text { N(4F)-C(20F)-C(21F)-C(22F) } & 119.2(4) \\ \text { C(24F)-C(20F)-C(21F)-C(22F) } & -1.9(5) \\ \text { C(20F)-C(21F)-C(22F)-C(23F) } & 28.0(5) \\ \text { C(21F)-C(22F)-C(23F)-C(24F) } & -43.7(5) \\ \text { C(22F)-C(23F)-C(24F)-C(25F) } & 162.7(4) \\ \text { C(22F)-C(23F)-C(24F)-C(20F) } & 41.9(5) \\ \text { N(4F)-C(20F)-C(24F)-C(23F) } & -145.5(4) \\ \text { C(21F)-C(20F)-C(24F)-C(23F) } & -24.5(5) \\ \text { N(4F)-C(20F)-C(24F)-C(25F) } & 90.3(4) \\ \text { C(21F)-C(20F)-C(24F)-C(25F) } & -148.7(4) \\ \text { C(26F)-N(5F)-C(25F)-O(6F) } & 4.7(6) \\ \text { C(26F)-N(5F)-C(25F)-C(24F) } & -177.7(4) \\ \text { C(23F)-C(24F)-C(25F)-O(6F) } & -21.9(6) \\ \text { C(20F)-C(24F)-C(25F)-O(6F) } & 95.2(5) \\ \text { C(23F)-C(24F)-C(25F)-N(5F) } & 160.5(4) \\ \text { C(20F)-C(24F)-C(25F)-N(5F) } & -82.3(5) \\ \text { C(25F)-N(5F)-C(26F)-C(28F) } & 70.8(5) \\ \text { C(25F)-N(5F)-C(26F)-C(29F) } & -52.7(5) \\ \text { C(25F)-N(5F)-C(26F)-C(27F) } & -169.3(4) \\ \text { C(30F)-N(6F)-C(29F)-O(7F) } & 2.7(7) \\ \text { C(30F)-N(6F)-C(29F)-C(26F) } & -176.5(4) \\ & \\ & \\ & \end{array}$


$\mathrm{N}(5 \mathrm{~F})-\mathrm{C}(26 \mathrm{~F})-\mathrm{C}(29 \mathrm{~F})-\mathrm{O}(7 \mathrm{~F})$

$\mathrm{C}(28 \mathrm{~F})-\mathrm{C}(26 \mathrm{~F})-\mathrm{C}(29 \mathrm{~F})-\mathrm{O}(7 \mathrm{~F})$

$\mathrm{C}(27 \mathrm{~F})-\mathrm{C}(26 \mathrm{~F})-\mathrm{C}(29 \mathrm{~F})-\mathrm{O}(7 \mathrm{~F})$

$\mathrm{N}(5 \mathrm{~F})-\mathrm{C}(26 \mathrm{~F})-\mathrm{C}(29 \mathrm{~F})-\mathrm{N}(6 \mathrm{~F})$

$\mathrm{C}(28 \mathrm{~F})-\mathrm{C}(26 \mathrm{~F})-\mathrm{C}(29 \mathrm{~F})-\mathrm{N}(6 \mathrm{~F})$

$\mathrm{C}(27 \mathrm{~F})-\mathrm{C}(26 \mathrm{~F})-\mathrm{C}(29 \mathrm{~F})-\mathrm{N}(6 \mathrm{~F})$

C(29F)-N(6F)-C(30F)-C(32F)

C (29F)-N(6F)-C(30F)-C(31F)

C(29F)-N(6F)-C(30F)-C(33F)

$\mathrm{C}(34 \mathrm{~F})-\mathrm{N}(7 \mathrm{~F})-\mathrm{C}(33 \mathrm{~F})-\mathrm{O}(8 \mathrm{~F})$

$\mathrm{C}(34 \mathrm{~F})-\mathrm{N}(7 \mathrm{~F})-\mathrm{C}(33 \mathrm{~F})-\mathrm{C}(30 \mathrm{~F})$

$\mathrm{N}(6 \mathrm{~F})-\mathrm{C}(30 \mathrm{~F})-\mathrm{C}(33 \mathrm{~F})-\mathrm{O}(8 \mathrm{~F})$

$\mathrm{C}(32 \mathrm{~F})-\mathrm{C}(30 \mathrm{~F})-\mathrm{C}(33 \mathrm{~F})-\mathrm{O}(8 \mathrm{~F})$

$\mathrm{C}(31 \mathrm{~F})-\mathrm{C}(30 \mathrm{~F})-\mathrm{C}(33 \mathrm{~F})-\mathrm{O}(8 \mathrm{~F})$

$\mathrm{N}(6 \mathrm{~F})-\mathrm{C}(30 \mathrm{~F})-\mathrm{C}(33 \mathrm{~F})-\mathrm{N}(7 \mathrm{~F})$

$\mathrm{C}(32 \mathrm{~F})-\mathrm{C}(30 \mathrm{~F})-\mathrm{C}(33 \mathrm{~F})-\mathrm{N}(7 \mathrm{~F})$

$\mathrm{C}(31 \mathrm{~F})-\mathrm{C}(30 \mathrm{~F})-\mathrm{C}(33 \mathrm{~F})-\mathrm{N}(7 \mathrm{~F})$

C(33F)-N(7F)-C(34F)-C(35F)

$\mathrm{C}(33 \mathrm{~F})-\mathrm{N}(7 \mathrm{~F})-\mathrm{C}(34 \mathrm{~F})-\mathrm{C}(38 \mathrm{~F})$

$\mathrm{N}(7 \mathrm{~F})-\mathrm{C}(34 \mathrm{~F})-\mathrm{C}(35 \mathrm{~F})-\mathrm{C}(36 \mathrm{~F})$

$\mathrm{C}(38 \mathrm{~F})-\mathrm{C}(34 \mathrm{~F})-\mathrm{C}(35 \mathrm{~F})-\mathrm{C}(36 \mathrm{~F})$

$\mathrm{C}(34 \mathrm{~F})-\mathrm{C}(35 \mathrm{~F})-\mathrm{C}(36 \mathrm{~F})-\mathrm{C}(37 \mathrm{~F})$

$\mathrm{C}(35 \mathrm{~F})-\mathrm{C}(36 \mathrm{~F})-\mathrm{C}(37 \mathrm{~F})-\mathrm{C}(38 \mathrm{~F})$

$$
\begin{gathered}
142.9(4) \\
18.9(6) \\
-99.5(5) \\
-37.8(6) \\
-161.8(4) \\
79.8(5) \\
57.9(6) \\
178.1(4) \\
-65.4(5) \\
2.6(7) \\
-171.9(4) \\
164.5(4) \\
40.1(6) \\
-78.9(5) \\
-20.8(6) \\
-145.2(4) \\
95.8(5) \\
89.6(5) \\
-155.4(4) \\
82.1(5) \\
-36.2(5) \\
43.9(5) \\
-34.2(5)
\end{gathered}
$$

136.4(4)

$-104.9(5)$

$-103.8(5)$

$14.8(5)$

$134.8(4)$

$12.1(5)$

$-1.6(8)$

$-179.4(5)$

$12.0(7)$

$-106.4(6)$

$-170.2(4)$

71.3(5)

$-132.8(5)$

122.3(6)

$-56.4(7)$

$0.9(9)$

179.7(6)

$0.1(10)$

$0.1(10)$

$-1.2(10)$

$-2.1(9)$

179.2(6)

2.3(10)

Symmetry transformations used to generate equivalent atoms: 
Table S6. Hydrogen bonds for $4 \quad\left[\AA\right.$ and $\left.^{\circ}\right]$.

\begin{tabular}{|c|c|c|c|c|}
\hline $\mathrm{D}-\mathrm{H} \ldots \mathrm{A}$ & $\mathrm{d}(\mathrm{D}-\mathrm{H})$ & $\mathrm{d}(\mathrm{H} \ldots \mathrm{A})$ & $d(D \ldots A)$ & $<(\mathrm{DHA})$ \\
\hline $\mathrm{N}(1)-\mathrm{H}(1 \mathrm{~A}) \ldots \mathrm{O}(7 \mathrm{D}) \# 1$ & 0.88 & 2.16 & $2.992(5)$ & 158.5 \\
\hline $\mathrm{N}(2)-\mathrm{H}(2 \mathrm{~A}) \ldots \mathrm{O}(8 \mathrm{E}) \# 1$ & 0.88 & 2.13 & $2.898(5)$ & 146.1 \\
\hline $\mathrm{N}(3)-\mathrm{H}(3 \mathrm{~A}) \ldots \mathrm{O}(2)$ & 0.88 & 2.08 & $2.889(5)$ & 152.2 \\
\hline $\mathrm{N}(4)-\mathrm{H}(4 \mathrm{~A}) \ldots \mathrm{O}(3)$ & 0.88 & 2.16 & $2.804(5)$ & 130.0 \\
\hline $\mathrm{N}(5)-\mathrm{H}(5 \mathrm{~A}) \ldots \mathrm{O}(4)$ & 0.88 & 2.08 & $2.954(5)$ & 176.1 \\
\hline $\mathrm{N}(6)-\mathrm{H}(6 \mathrm{~A}) \ldots \mathrm{O}(5)$ & 0.88 & 2.22 & $3.063(5)$ & 160.8 \\
\hline $\mathrm{N}(7)-\mathrm{H}(7 \mathrm{~A}) \ldots \mathrm{O}(6)$ & 0.88 & 2.02 & $2.878(5)$ & 164.6 \\
\hline $\mathrm{N}(1 \mathrm{~A})-\mathrm{H}(1 \mathrm{AA}) \ldots \mathrm{O}(7 \mathrm{E}) \# 2$ & 0.88 & 2.21 & $3.062(5)$ & 162.3 \\
\hline $\mathrm{N}(2 \mathrm{~A})-\mathrm{H}(2 \mathrm{AA}) \ldots \mathrm{O}(8 \mathrm{~F}) \# 2$ & 0.88 & 2.07 & $2.908(5)$ & 158.8 \\
\hline $\mathrm{N}(3 \mathrm{~A})-\mathrm{H}(3 \mathrm{AA}) \ldots \mathrm{O}(2 \mathrm{~A})$ & 0.88 & 2.03 & $2.895(5)$ & 166.3 \\
\hline $\mathrm{N}(4 \mathrm{~A})-\mathrm{H}(4 \mathrm{AA}) \ldots \mathrm{O}(3 \mathrm{~A})$ & 0.88 & 2.12 & $2.836(5)$ & 138.2 \\
\hline $\mathrm{N}(5 \mathrm{~A})-\mathrm{H}(5 \mathrm{AA}) \ldots \mathrm{O}(4 \mathrm{~A})$ & 0.88 & 2.07 & $2.949(5)$ & 172.7 \\
\hline $\mathrm{N}(6 \mathrm{~A})-\mathrm{H}(6 \mathrm{AA}) \ldots \mathrm{O}(5 \mathrm{~A})$ & 0.88 & 2.10 & $2.965(5)$ & 166.2 \\
\hline $\mathrm{N}(7 \mathrm{~A})-\mathrm{H}(7 \mathrm{AA}) \ldots \mathrm{O}(6 \mathrm{~A})$ & 0.88 & 2.08 & $2.916(5)$ & 158.8 \\
\hline $\mathrm{N}(1 \mathrm{~B})-\mathrm{H}(1 \mathrm{BA}) \ldots \mathrm{O}(7 \mathrm{~F}) \# 2$ & 0.88 & 2.13 & $2.961(5)$ & 158.2 \\
\hline $\mathrm{N}(2 \mathrm{~B})-\mathrm{H}(2 \mathrm{BA}) \ldots \mathrm{O}(8) \# 3$ & 0.88 & 2.04 & $2.876(5)$ & 157.5 \\
\hline $\mathrm{N}(3 \mathrm{~B})-\mathrm{H}(3 \mathrm{BA}) \ldots \mathrm{O}(2 \mathrm{~B})$ & 0.88 & 2.05 & $2.887(5)$ & 159.5 \\
\hline $\mathrm{N}(4 \mathrm{~B})-\mathrm{H}(4 \mathrm{BA}) \ldots \mathrm{O}(3 \mathrm{~B})$ & 0.88 & 2.09 & $2.843(5)$ & 142.9 \\
\hline $\mathrm{N}(5 \mathrm{~B})-\mathrm{H}(5 \mathrm{BA}) \ldots \mathrm{O}(4 \mathrm{~B})$ & 0.88 & 2.06 & $2.930(5)$ & 170.7 \\
\hline $\mathrm{N}(6 \mathrm{~B})-\mathrm{H}(6 \mathrm{BA}) \ldots \mathrm{O}(5 \mathrm{~B})$ & 0.88 & 2.12 & $2.971(5)$ & 161.2 \\
\hline N(7B)-H(7BA) ...O(6B) & 0.88 & 2.08 & $2.932(5)$ & 161.9 \\
\hline $\mathrm{N}(1 \mathrm{C})-\mathrm{H}(1 \mathrm{CA}) \ldots \mathrm{O}(7) \# 3$ & 0.88 & 2.29 & $3.073(5)$ & 148.1 \\
\hline $\mathrm{N}(2 \mathrm{C})-\mathrm{H}(2 \mathrm{CA}) \ldots \mathrm{O}(8 \mathrm{~A}) \# 4$ & 0.88 & 2.19 & $2.905(5)$ & 138.5 \\
\hline $\mathrm{N}(3 \mathrm{C})-\mathrm{H}(3 \mathrm{CA}) \ldots \mathrm{O}(2 \mathrm{C})$ & 0.88 & 2.00 & $2.835(5)$ & 159.0 \\
\hline $\mathrm{N}(4 \mathrm{C})-\mathrm{H}(4 \mathrm{CA}) \ldots \mathrm{O}(3 \mathrm{C})$ & 0.88 & 2.02 & $2.817(5)$ & 149.5 \\
\hline $\mathrm{N}(5 \mathrm{C})-\mathrm{H}(5 \mathrm{CA}) \ldots \mathrm{O}(4 \mathrm{C})$ & 0.88 & 2.08 & $2.953(5)$ & 171.2 \\
\hline $\mathrm{N}(6 \mathrm{C})-\mathrm{H}(6 \mathrm{CA}) \ldots \mathrm{O}(5 \mathrm{C})$ & 0.88 & 2.05 & $2.911(5)$ & 165.3 \\
\hline $\mathrm{N}(7 \mathrm{C})-\mathrm{H}(7 \mathrm{CA}) \ldots \mathrm{O}(6 \mathrm{C})$ & 0.88 & 2.07 & $2.914(5)$ & 161.2 \\
\hline $\mathrm{N}(1 \mathrm{D})-\mathrm{H}(1 \mathrm{DA}) \ldots \mathrm{O}(7 \mathrm{~A})$ & 0.88 & 2.12 & $2.970(5)$ & 163.5 \\
\hline $\mathrm{N}(2 \mathrm{D})-\mathrm{H}(2 \mathrm{DA}) \ldots \mathrm{O}(8 \mathrm{~B})$ & 0.88 & 2.06 & $2.874(5)$ & 153.4 \\
\hline $\mathrm{N}(3 \mathrm{D})-\mathrm{H}(3 \mathrm{DA}) \ldots \mathrm{O}(2 \mathrm{D})$ & 0.88 & 2.03 & $2.863(5)$ & 157.6 \\
\hline $\mathrm{N}(4 \mathrm{D})-\mathrm{H}(4 \mathrm{DA}) \ldots \mathrm{O}(3 \mathrm{D})$ & 0.88 & 2.11 & $2.845(5)$ & 140.2 \\
\hline $\mathrm{N}(5 \mathrm{D})-\mathrm{H}(5 \mathrm{DA}) \ldots \mathrm{O}(4 \mathrm{D})$ & 0.88 & 2.06 & $2.929(5)$ & 170.9 \\
\hline $\mathrm{N}(6 \mathrm{D})-\mathrm{H}(6 \mathrm{DA}) \ldots \mathrm{O}(5 \mathrm{D})$ & 0.88 & 2.11 & $2.971(5)$ & 164.5 \\
\hline $\mathrm{N}(7 \mathrm{D})-\mathrm{H}(7 \mathrm{DA}) \ldots \mathrm{O}(6 \mathrm{D})$ & 0.88 & 2.06 & $2.900(5)$ & 158.9 \\
\hline N(1E)-H(1EA)...O(7B) & 0.88 & 2.30 & $3.071(5)$ & 145.9 \\
\hline $\mathrm{N}(2 \mathrm{E})-\mathrm{H}(2 \mathrm{EA}) \ldots \mathrm{O}(8 \mathrm{C})$ & 0.88 & 2.10 & $2.808(5)$ & 136.4 \\
\hline $\mathrm{N}(3 \mathrm{E})-\mathrm{H}(3 \mathrm{EA}) \ldots \mathrm{O}(2 \mathrm{E})$ & 0.88 & 2.05 & $2.899(5)$ & 161.5 \\
\hline $\mathrm{N}(4 \mathrm{E})-\mathrm{H}(4 \mathrm{EA}) \ldots \mathrm{O}(3 \mathrm{E})$ & 0.88 & 2.19 & $2.890(5)$ & 136.6 \\
\hline N(5E)-H(5EA)...O(4E) & 0.88 & 2.08 & $2.945(5)$ & 168.5 \\
\hline $\mathrm{N}(6 \mathrm{E})-\mathrm{H}(6 \mathrm{EA}) \ldots \mathrm{O}(5 \mathrm{E})$ & 0.88 & 2.27 & $3.125(5)$ & 165.6 \\
\hline N(7E)-H(7EA)...O(6E) & 0.88 & 2.07 & $2.910(5)$ & 159.9 \\
\hline $\mathrm{N}(1 \mathrm{~F})-\mathrm{H}(1 \mathrm{FA}) \ldots \mathrm{O}(7 \mathrm{C})$ & 0.88 & 2.06 & $2.918(5)$ & 165.8 \\
\hline $\mathrm{N}(2 \mathrm{~F})-\mathrm{H}(2 \mathrm{FA}) \ldots \mathrm{O}(8 \mathrm{D}) \# 4$ & 0.88 & 2.07 & $2.903(5)$ & 158.8 \\
\hline $\mathrm{N}(3 \mathrm{~F})-\mathrm{H}(3 \mathrm{FA}) \ldots \mathrm{O}(2 \mathrm{~F})$ & 0.88 & 2.05 & $2.897(5)$ & 161.7 \\
\hline $\mathrm{N}(4 \mathrm{~F})-\mathrm{H}(4 \mathrm{FA}) \ldots \mathrm{O}(3 \mathrm{~F})$ & 0.88 & 2.14 & $2.835(5)$ & 135.0 \\
\hline
\end{tabular}




$\begin{array}{lllll}\mathrm{N}(5 \mathrm{~F})-\mathrm{H}(5 \mathrm{FA}) \ldots \mathrm{O}(4 \mathrm{~F}) & 0.88 & 2.06 & 2.934(5) & 170.4 \\ \mathrm{~N}(6 \mathrm{~F})-\mathrm{H}(6 \mathrm{FA}) \ldots \mathrm{O}(5 \mathrm{~F}) & 0.88 & 2.15 & 3.010(5) & 167.4 \\ \mathrm{~N}(7 \mathrm{~F})-\mathrm{H}(7 \mathrm{FA}) \ldots \mathrm{O}(6 \mathrm{~F}) & 0.88 & 2.09 & 2.932(5) & 159.6\end{array}$

Symmetry transformations used to generate equivalent atoms:

\#1 $\mathrm{x}-1, \mathrm{y}-1, \mathrm{z} \quad \# 2 \mathrm{x}-1, \mathrm{y}, \mathrm{z}+1 \quad \# 3 \mathrm{x}, \mathrm{y}, \mathrm{z}+1 \quad$ \#4 $\mathrm{x}, \mathrm{y}-1, \mathrm{z}$ 